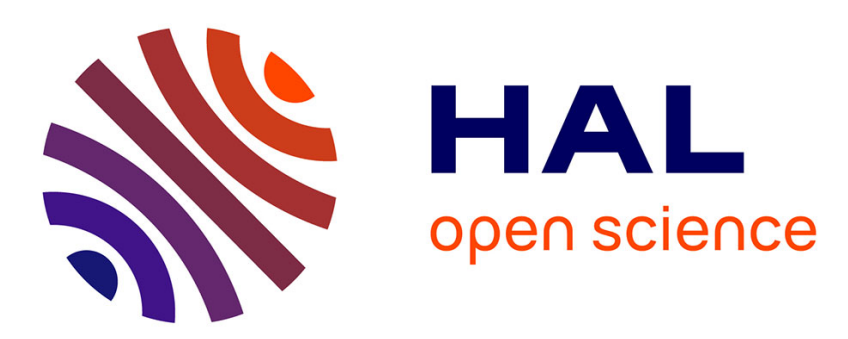

\title{
A Green operator-based elastic modeling for two-phase pantographic-inspired bi-continuous materials
}

\author{
M. Spagnuolo, P. Franciosi, F. Dell'Isola
}

\section{To cite this version:}

M. Spagnuolo, P. Franciosi, F. Dell'Isola. A Green operator-based elastic modeling for two-phase pantographic-inspired bi-continuous materials. International Journal of Solids and Structures, 2020, 188-189, pp.282-308. 10.1016/j.ijsolstr.2019.10.018 . hal-02915323

\section{HAL Id: hal-02915323 \\ https://hal.science/hal-02915323}

Submitted on 14 Aug 2020

HAL is a multi-disciplinary open access archive for the deposit and dissemination of scientific research documents, whether they are published or not. The documents may come from teaching and research institutions in France or abroad, or from public or private research centers.
L'archive ouverte pluridisciplinaire HAL, est destinée au dépôt et à la diffusion de documents scientifiques de niveau recherche, publiés ou non, émanant des établissements d'enseignement et de recherche français ou étrangers, des laboratoires publics ou privés. 


\title{
A Green operator-based elastic modeling for two-phase pantographic-inspired bi-continuous materials
}

\author{
M. Spagnuolo ${ }^{\mathrm{a}, \mathrm{b}}$, P. Franciosi ${ }^{\mathrm{a}, *}$, F. dell'Isola ${ }^{\mathrm{b}, \mathrm{c}}$ \\ a CNRS, LSPM UPR3407, Université Paris 13, France \\ ${ }^{\mathrm{b}}$ MEMoCS Università dell'Aquila, Roma, Italy \\ ' Università di Roma “La Sapienza", Roma, Italy
}

\begin{abstract}
A B S T R A C T
We propose an estimate for the effective elastic properties of a new imagined two-phase bi-continuous composite material type with a so-called "pantographic-inspired" (P-I) architecture in the sense of a matrix reinforcement which is a 3D fiber network capable of large, pantographic-like, deformations, owing to particular properties carried by the fiber interconnections. Such fiber networks co-continuous with the matrix are described from an assemblage of inter-penetrated planar alignments of parallel infinite, identical and equally distant, rods or beams that we call fiber layers or FPAs for "fiber planar alignments". Piece-wise linear deformation of such a structure is constrained, as 2D pantographs are, by the specific relations that link the evolutions of the FPA misorientations, of the fiber inter distance and concentration within each FPA, and of the given characteristics to the through-layer interconnections. We derive effective properties within a well defined first gradient elastic homogenization scheme for macrohomogeneous composite structures, based on explicating the involved mean Green operator ( $\mathrm{mGO}$ ) for the representative pattern of such a P-I networked reinforcement in an isotropic matrix. This mGO for P-I composites is built in making use of previously derived and presented mGOs for rod and beam FPAs. Comparisons with Young and shear modulus variations during numerical homogeneous extension simulations show that the proposed modeling provides relevant effective property evolutions with the advantage of being analytical. Comparing so estimated force-displacement curves with numerical ones for 2D pantographs enables to identify ways to further account in the modeling for the specific strengthening effects of the FPA interconnections in pantographs. This tends to prove such P-I composite structures, still to be manufactured, to possibly also behave similarly to pantograph ones.
\end{abstract}

\section{Introduction}

Pantographic structures which are capable of large elastic deformation at low energy expense are promising meta-materials potentially of concern and interest in many domains, from functional devices as actuators and sensors to geo-mechanical, biomechanical or aerospace applications, for only mentioning a few. Basically introduced in the 19th century as deployable 2D or 3D structures (Wallace, 1836; Selling, 1887), they are nowadays also of primary importance for fundamental purposes regarding the study of generalized continua and, in the specific, of second gradient materials (Pideri and Seppecher, 1997; dell'Isola and Seppecher, 1997; dell'Isola et al., 2015; Alibert et al., 2003; Seppecher et al., 2011; Misra and Poorsolhjouy, 2015; Eremeyev, 2016; Eremeyev et al.,

\footnotetext{
* Corresponding author

E-mail address: patrick.franciosi@univ-paris13.fr (P. Franciosi).
}

2016, 2018; Cuomo et al., 2017; Placidi et al., 2018, 2019; Barchiesi and Khakalo, 2019).

Here, restricting our attention to the homogeneous behavior range of these structures, the concern is to determine effective elastic-like properties for two-phase materials that comprise an isotropic compliant matrix and a 3D fiber pantographicinspired (P-I) network co-continuous with the embedding matrix. This phase assemblage with expectedly retained potentialities of large deformation at low energy cost is a newly imagined type of bi-continuous composites or composites with interpenetrating phases, the variety of which is already quite large since it non exhaustively assembles structures as different as open cell foams (Roberts and Garboczi, 2002; Gong et al., 2005), bones (Kinney et al., 2005), woven textiles (Udhayaraman and Mulay, 2017), metal or polymer mixtures (Peng et al., 2001; Veenstra et al., 2000; Clyne et al., 2005; Leclerc et al., 2016), weakly compacted powders (Poquillon et al., 2002) or partially melt alloys (Limodin et al., 2007). An example of such a new P-I 

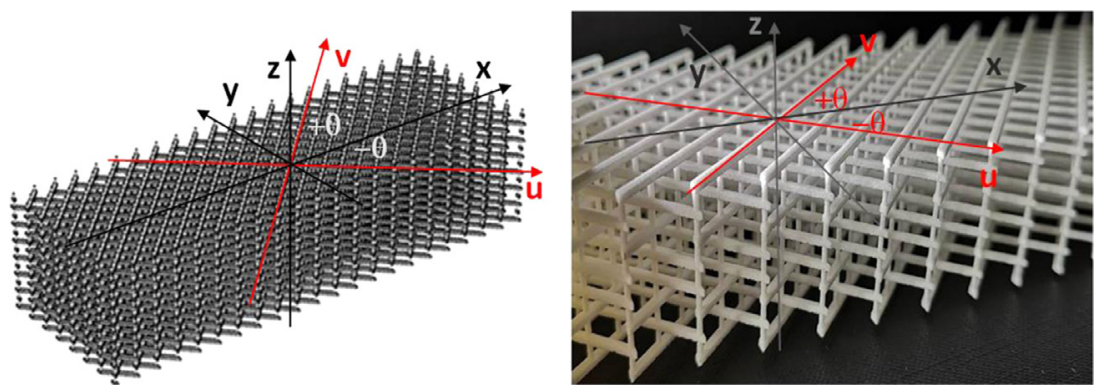

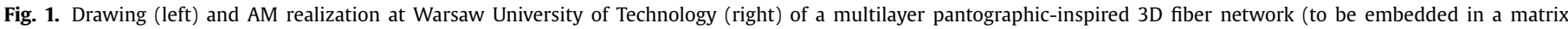
not visible on the figure).

Table 1

Definitions of abbreviations and acronyms.

\begin{tabular}{ll}
\hline Abbreviations and acronisms & Definitions \\
\hline $\mathrm{FPA}(\mathrm{s})$ & fiber planar alignment(s) \\
$P-I$ & pantographic-inspired \\
$L-R, R-L$ & Left-Right, Right-Left alternated piling of FPAs \\
$\mathrm{GO}(\mathrm{s}), \mathrm{mGO}(\mathrm{s})$ & Green operator(s), mean Green operator(s) \\
C-fibers & cylindrical fibers with circular cross section \\
SQ-beams & beams with square cross section \\
$R T, I R T$ & Radon transform, Inverse Radon transform \\
$P C W$ & Ponte-Castaneda and Willis effective property estimates (1995) \\
\hline
\end{tabular}

Table 2

Definitions of main variables and notations.

\begin{tabular}{|c|c|}
\hline Variable and notation & Definitions \\
\hline$V ; \boldsymbol{r}$ & A domain in the $R^{3}$ infinite medium; a point in $V$ or in $R^{3}$ \\
\hline $\boldsymbol{E}^{\boldsymbol{V}}(\boldsymbol{r}) ; \overline{\boldsymbol{E}^{\boldsymbol{V}}}$ or $\boldsymbol{E}^{\boldsymbol{V}}$ & The Eshelby (rank 4 or rank 2) tensor at point $\boldsymbol{r}=\left(r_{1}, r_{2}, r_{3}\right)$ in $V$; the mean or the uniform Eshelby tensor over all $\boldsymbol{r}$ points of $V$ \\
\hline $\boldsymbol{t}^{\mathrm{V}}(\boldsymbol{r}) ; \overline{\boldsymbol{t}^{\mathbf{V}}}$ or $\boldsymbol{t}^{\mathrm{V}}$ & The GO (rank 4 or rank 2 ) at point $\boldsymbol{r}$ in $V$; the mean or the uniform GO over all $\boldsymbol{r}$ points of $V$ \\
\hline$C ; C^{l}$ or $\boldsymbol{C}^{M}$ & The elastic-like rank 4 property tensor for a homogeneous medium; for a homogeneous specific phase I or M \\
\hline$\psi_{V}(\omega, \boldsymbol{r}) ; \overline{\psi_{V}(\omega)}$ & The shape function at point $\boldsymbol{r}$ in $V$; the mean shape function of $V$ \\
\hline$\overline{\psi_{A p a t t e r n(B)}(\omega)}$ & The mean shape function for some included pattern in the infinite medium with A,B specified pattern characteristics \\
\hline$\psi_{\text {int }(A p a t t e r n(B))}(\omega)$ & The interaction part of the mean shape function for some included pattern in the infinite medium with $A, B$ pattern characteristics \\
\hline $\boldsymbol{t}^{\text {Apattern }(B)}$ & The mGO for some included pattern in the infinite medium with A,B specified pattern characteristics \\
\hline$\overline{t^{\text {int }(A p a t t e r n(B))}}$ & The interaction part of the mGO for some included pattern in the infinite medium with $A, B$ specified pattern characteristics \\
\hline$C_{\text {effM }}^{A \text { pattern }(B) / D}$, & $\begin{array}{l}\text { The effective elastic property tensor from the } M \text { estimate model for an infinite medium reinforced with the second phase pattern } \\
\text { of A,B,D characteristics }\end{array}$ \\
\hline$T_{i j k l}^{X X X} ; T_{i j}^{X X X}$ & The components of $\boldsymbol{T}^{\mathrm{XXX}}$ tensor/operator when of rank 4; of rank-2 \\
\hline
\end{tabular}

internal composite structure (the matrix is made invisible) in that diversity is shown in Fig. 1 (left as a drawing and right as a picture of additive manufacturing realization ${ }^{1}$ ), for the case when the fiber network symmetry is orthogonal, what would in general correspond to a reference undeformed situation. The Fig. 1 anticipates on the fact that, in the main cases of this new composite type that we examine here, one of the 3 (initially orthogonal) fiber families differs (the $\boldsymbol{z}$-oriented one as indicated) and is treated differently from the two other ones which keep identical (and identically evolving) characteristics, and in particular their two opposite orientation angles $\pm \theta$ with regard to some in-layer direction $\boldsymbol{x}$, as detailed in the following.

All the abbreviation and acronym definitions appearing along this text are collected in Table 1; similarly, the definitions of the main variables and notations can be found in Table 2. This fiber structure, to be matrix-embedded for building a P-I composite, can be seen as a particular sub-class among more general 3D fiber networked structures where all three constitutive fiber families could in principle have either different characteristics or identical ones and could play either different or similar roles, in a sort of general "triclinic" network in the sense, without any reference to crystal structures, of being currently (that is possibly evolving under

\footnotetext{
${ }^{1}$ By courtesy of Prof. T. Lekszycki, Warsaw University of Technology.
}

deformation) characterized by a representative parallelepiped-like volume with three unequal sides and three unequal angles. This is tentatively represented in Fig. 2 by one (left) and 4 (middle) elementary volume element(s). The 3 thick bi-colored edges on this representative volume, to be seen as unequal, figure the 3 inter penetrating fiber families. Each family pair belongs to a same family of parallel planes with alternated orientations (as the yellowblue and the yellow-red ones along $\mathbf{v}(\theta)$ and $\mathbf{u}\left(-\theta^{\prime}\right)$ directions, with $0<\theta^{\prime} \neq \theta$, in the $\boldsymbol{x}-\boldsymbol{y}$ plane), while the third ( $\boldsymbol{w}$ say) family goes through these layers. With some periodicity, some of the fibers in each 3 family need be layer-inter-connecting (the thin dashed lines) to ensure more or less continuity and cohesion to the network. Hence, many such structures can be thought as basically made of three inter-penetrated and inter-connected assemblages of parallel fibers under the form of either fiber planar alignments (to be denoted FPAs for short) or 1D bundles which can also possibly seen in turn as assemblages of coaxial FPAs. On the Fig. 2, the connections between fibers of different orientations are symbolized by "hinges" (black open circles) which hide the details of the operating mechanism for deforming the network, a point not to be entered here for it will be shown that only the geometrical and related stiffness descriptors of these connections need be specified for the formulation of P-I constitutive equations.

Going back to the specific P-I networks shown in Fig. 1 that constitute the matrix-embedded phase in the examined P-I 

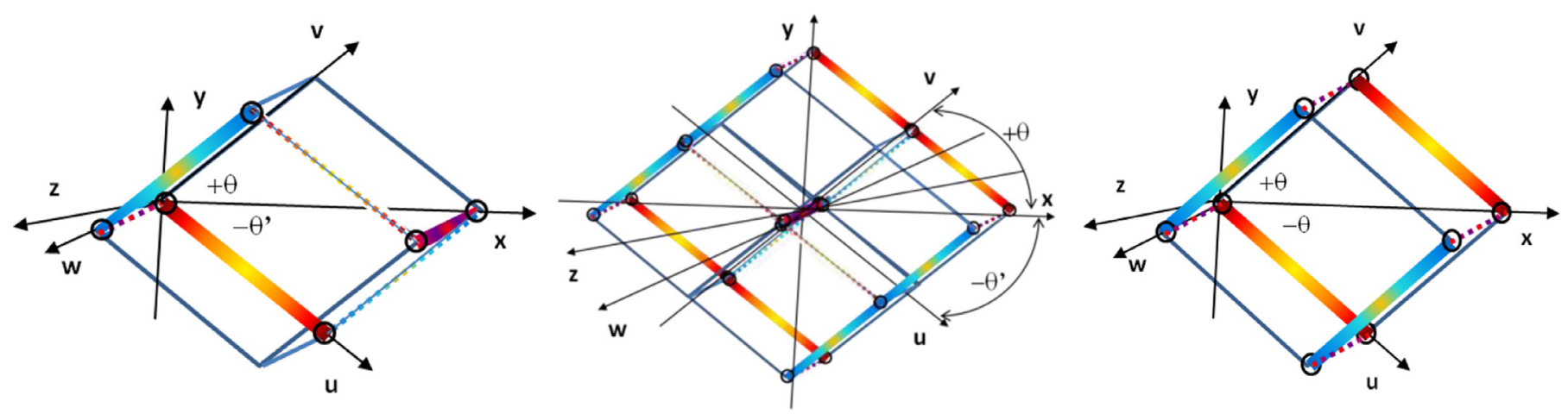

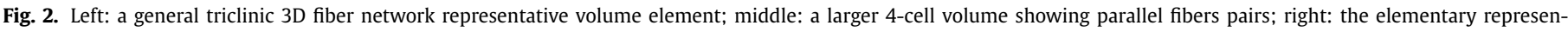
tative volume corresponding to the structures of Fig. 1 shown as a particular case of the general one.
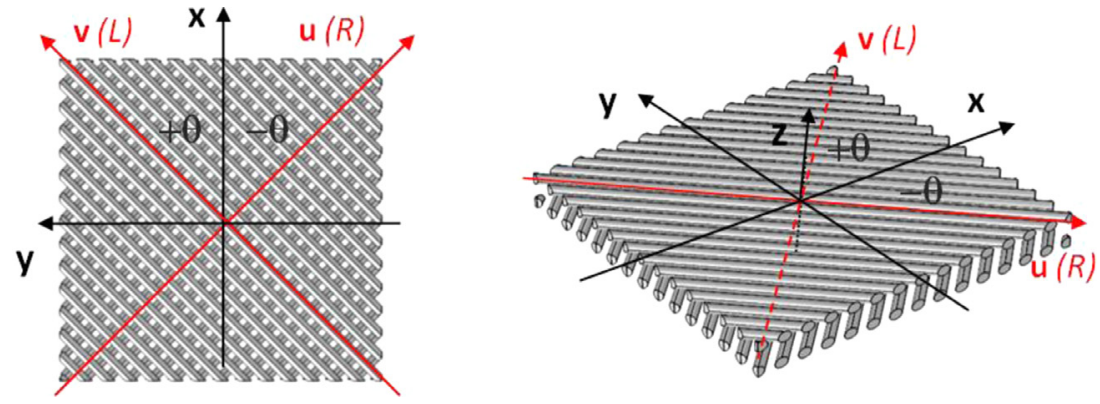

Fig. 3. Representation of an alternated FPA pair constituting the typical pantographic-inspired bi-layer (no interconnections are visible).

composites (this embedding matrix is made invisible on most of the Figures to come), only considering here fiber-like elements which are either of circular (rods) or of square (beams) cross sections, we reduce the examined situations to cases when the FPA assemblages are alternately $L-R$ (for left and right) and symmetrically $( \pm \theta)$ oriented layers in one ( $\boldsymbol{x}$-y say) plane, with regard to one (here $\boldsymbol{x}$ ) direction. From such a structure symmetry assumption, the element (fiber) inter-distance is kept identical for the two FPA families which are parallel to the $\boldsymbol{x}-\boldsymbol{y}$ plane and the FPAs are assumed to remain planar and parallel (neither out-ofplane twist nor flexion of the FPAs are considered). Thus, the unit pantographic-like constitutive domain of the structure is an alternated $L-R$ (or $R-L$ ) pair of contiguous FPAs, as exemplified in Fig. 2 right as a particular case of the general described family which in the third ( $\boldsymbol{w}$ or $\boldsymbol{z}$ ) direction has only inter-connecting elements and more simply in Fig. 3 in the orthogonal reference case $(\theta=\pi / 4)$, with a layer inter distance not necessarily equal to the in-layer fiber inter distance. Thus, the all identical rod or beam elements in the successive layers which are respectively oriented along some $\mathbf{u}$ and $\mathbf{v}$ oppositely varying directions, are currently $2 \theta-$ misoriented (note that for the same symmetry reason that makes $L-R$ and $R$-Lpilings equivalent, it is equivalent to alternate the layers downwards or upwards along the $\boldsymbol{z}$ normal to the $\boldsymbol{x}$ - $\boldsymbol{y}$ plane). The inter connecting hinges are taken to be, in the average, aligned along that third family of $\boldsymbol{w}$-oriented fiber-like elements of the 3D network which may a priori be inclined on the $\boldsymbol{z}$ axis. It is worthy to keep from now in mind for the final discussion, that variations of the $2 \theta$ misorientation angle between successive $(L, R, L$, ...) parallel FPAs can also be related to torsion and bending (in the sense of inclination) along the third interconnecting element family, and hence related to some torsion and bending aptitude for these swiveling interconnections. In a first step, these features are disregarded. In contrast, the embedding compliant matrix will be kept homogeneous that is local network-due distortions are ignored.
We mainly consider the in-plane $(\boldsymbol{x}-\boldsymbol{y})$ homogeneous extension, in direction $\boldsymbol{x}$ say, of a matrix reinforced by such a network, the overall in-plane behavior of 2D pantographs during extension or shear being known to be in first approximation piece-wise homogeneous extension (Placidi et al., 2016; Scerrato et al., 2016a; dell'Isola et al., 2016, 2017; Turco et al., 2016a, 2017; Greco et al., 2017; Barchiesi et al., 2018, 2019a; Placidi et al., 2017; Laudato et al., 2018; De Angelo et al., 2019a), each homogeneous domain possibly supporting additionally a specific body rotation with regard to the main axis, as illustrated in Fig. 4 (where identically colored fibers are those belonging to a same - L or $R$ - FPA in any contiguous pair). As shown in this Fig. 4, apart from the end domains (as NMP, RTU) which in first approximation do not deform, each sub-domain homogeneous straining corresponds to an equal and opposite variation of angles $-\theta=(\mathbf{x}, \mathbf{u})$ and $\theta=(\mathbf{x}, \mathbf{v})$, which are related to an evolution of a same element inter-distance in the two FPA families. A shear $\boldsymbol{x} / \boldsymbol{y}$ (or $\boldsymbol{y} / \boldsymbol{x}$ ) on such a pantographic structure also corresponds to similar piece-wise homogeneous extensions of sub domains (as the central pantograph part $P Q R S$ in Fig. 4 right), with the extension direction being additionally inplane rotated (from $\boldsymbol{z}$ to $\boldsymbol{z}$, as drawn). Lateral domains also deform by combining extension and rotation. Simple angular relations between sub-domains are collected in Appendix A for the case of the "short" pantograph of Fig. 4, in both axial and shear in-plane deformation. It is also noteworthy that specific end conditions for clamping the fiber layers when applying axial extension on the structure can suppress the end and lateral sub-domains and yield an overall homogeneous extension.

The exemplified configuration in Figs. 3 and 4 keeps the through-layers connecting third fiber bundle normal to the FPAs. This corresponds to the one-placement-field assumption frequently used for the strength of the layer interconnections (Boutin et al., 2017; Barchiesi and Placidi, 2017; Placidi et al., 2016; Giorgio, 2016; Yang et al., 2018; Giorgio et al., 2017; Turco et al., 2016b; Scerrato et al., 2016b; Yang and Müller, 2019), while inclinable 

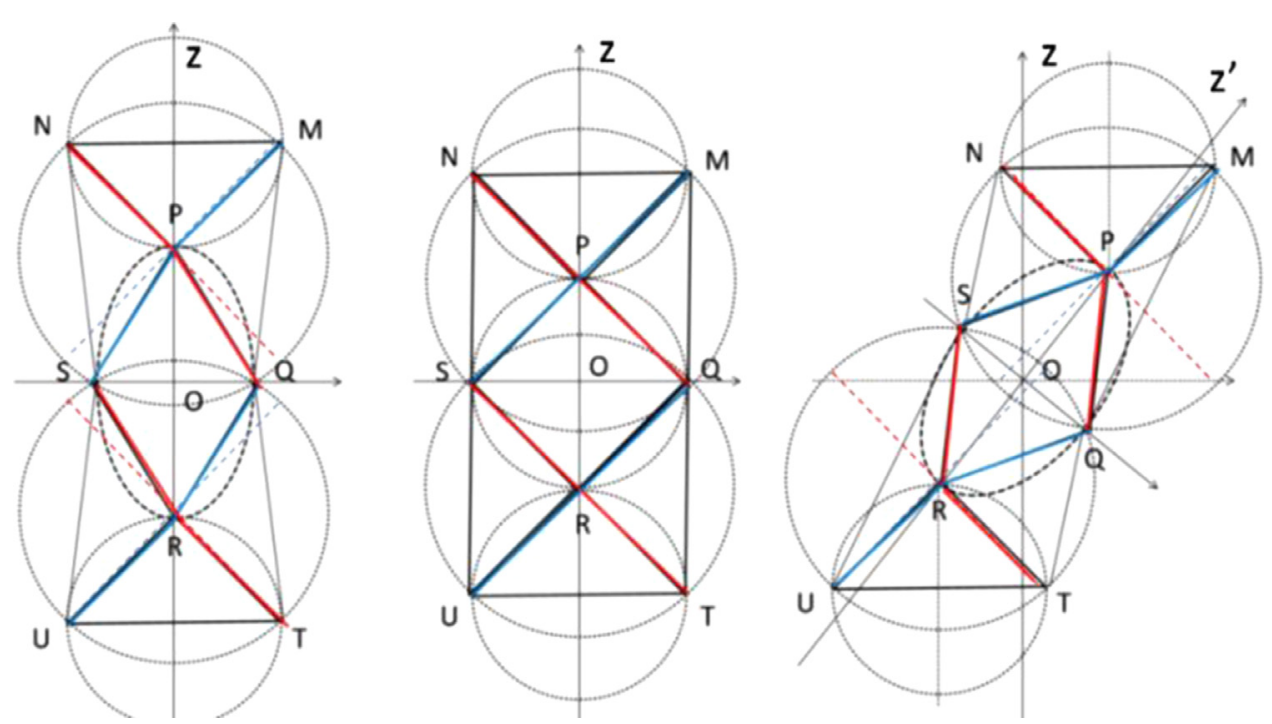

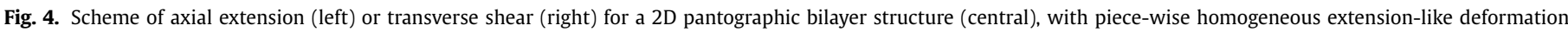
of sub-domains PQRS, MPQ = SRU, NPS = QRT, with rotations. Fiber colors indicate L or R planar alignments (PAs). Interconnecting pivots are normal to the FPAs.

interconnections would correspond to a two-placement-field assumption as discussed in Spagnuolo et al. (2017) and more generally to a currently triclinic network structure as the one of Fig. 2 left and middle. As is also observed on the simple 2D views of Fig. 4, the homogeneously deformed sub-domains are in-plane connected along more or less extended boundary zones where second gradient effects are localized, the nature of which is related to a local in-plane bending of the aligned fibers. These singularities are disregarded in the present work.

Hence, the piece-wise homogeneously deformed P-I fiber network of concern is assumed to be embedded in a compliant matrix which is also assumed to deform piece-wise homogeneously and with homogeneous sub-domains of the two phases being coincident. Consequently, the overall (effective) behavior of such a composite structure can be approached, as far as attention is paid to homogeneous applied deformation (that is at the scale of the homogeneously deforming sub-domains in first approximation), in using homogenization frameworks applicable to reinforced matrices, with the two phase co-continuity deserving some particular considerations as addressed in details in Franciosi $(2012,2013)$ and Franciosi and Charles (2016). The embedded fiber network being considered as substantially deformable, its representative pattern, although taken to remain piece-wise homogeneous, is expected to not remain invariant along any deformation path and so it is as well then for the effective elastic properties of the composite structure in each such homogeneous domain. If one further disregards the damage features possibly related to debonding between the fibers and the matrix, as well as to the matrix or fiber fracture, the major issue to estimate the current effective properties of such composites from a homogenization procedure is to well enough describe the reinforcing networked fiber pattern in terms of its so called mean Green operator (mGO) and to have an expression of easy use to follow at the best the expectedly large variations of this operator with the pattern structure evolution under possibly large homogeneous extension.

In the case when the fibers are infinite cylinders with circular cross section of same radius $\mathrm{R}$ (called C-fibers), global mGOs for C-fiber FPAs of $n$ (up to infinite) parallel, identical and equally distant, elements as exemplified in Fig. 5 were calculated in Franciosi et al. (2019), for all fiber axis-to-axis inter distance values 2L from infinity to contact, with all fiber pair interaction accounted for in the $n$-FPAs up to infinite $n$ value, for the case of an embedding medium with isotropic, elastic or dielectric, properties. These
mGOs for $n$-FPAs of C-fibers in an isotropic matrix were shown, as a result of non trivial and tedious calculations, to have a simple expression, with application examples given in the cited reference for estimating variations of effective stiffness properties of 1D fiber bundles when compressed or extended transversally with regard to the fiber direction. Similar mGOs were calculated more easily in Franciosi (2018) for $n$-FPAs of parallel beams with various polygonal, and in particular rectangular (including square), cross sections, for any fiber inter distance as well, the exact form of which being not reducible to a simple one as for the C-fiber $n$-FPAs. A first new result provided in the present work is an accurate approximation, from graphical analysis, of easier use for the mGO of infinite FPAs of SQ-beams, square cross sections being close to the frequently rectangular ones realized in $3 \mathrm{D}$ printing elaboration as exemplified in Fig. 1 right.

As for all inclusion patterns, the mGOs of FPAs share into a main term and a global pair-interactions contribution. On the geometrical ground, the main term only depends on the individual element shape(s) and the interaction part depends on the relative positions and distances for all element pairs in the pattern, that is of the inter-distance(s) between parallel elements in FPAs. ${ }^{2}$ In having, in the cited references, considered FPAs up to an infinite number of elements, it was shown that the critical inter distance above which a fiber or a beam can be considered as isolated was typically (depending on the wished precision) ten to twenty times the C-fiber radius or the SQ-beam side. This length defines an influence zone around any element out of which the rest of the alignment does not contribute much to the interaction and thus to the pattern mGO. This limited influence zone conversely allows the use of the mGO of infinite patterns as soon as the element number is large enough in a FP, that is above ten fibers typically. The knowledge of this mGO for infinite FPAs of C-fibers or of SQ-beams was thus the very first necessary information to attempt performing the homogenization-based estimate of effective properties for the two-phase P-I structure type here of concern. The present work could not be realized without having first solved these FPA mGO problems for circular cylinders and square beams in the two cited references eventhough the previously achieved determinations of these C-fiber and SQ-beam FPA mGOs also constituted results of

\footnotetext{
2 Interactions between not parallel fibers are localized "around" the shortest distance between the two fibers. They are disregarded in the modeling for sake of simplifying, but their effect will be estimated from computational simulations.
} 

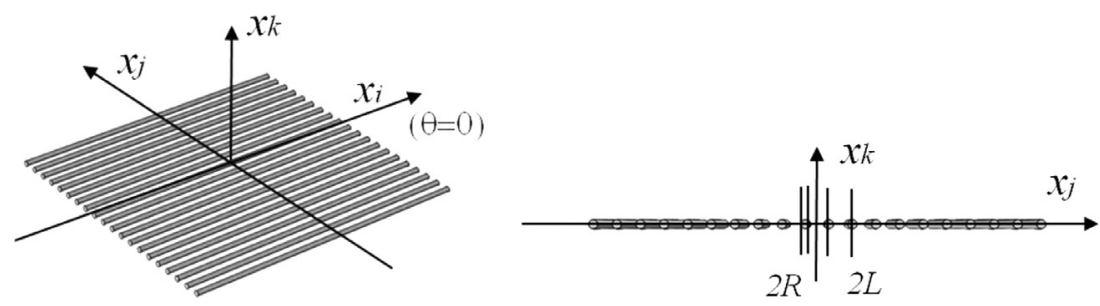

Fig. 5. Representation of a single FPA of C-fibers with radius $R$ and axis to axis inter distance 2L.

own interest for varieties of applications. Only these obtained mGO forms will be recalled here, with a brief summary of the calculation routes.

The second necessary information for the composite deformation to be "pantographic-inspired", that is to be ruled by the P-I network deformation characteristics, is to well enough describe the through-layer interconnections for this description will be taken to drive the in-layer fiber network behavior under straining and hence the one of the P-I composite. This amounts in the one hand to well enough accounting for the through-layer stiffness of the FPA assemblage and on the other hand to describe the in-plane relations between the fiber network extension, the fiber interdistances in the FPAs, the misorientation angle $2 \theta$ between the alternated FPAs, and the volume fraction changes for the fiber network in the matrix. The chosen description must represent the specific characteristics which are carried by the inter-connections between the elements of the piled FPAs in the assemblage, called pivots at the scale of each two consecutive $L-R o r \quad R-L$ layer pairs as exemplified in Fig. 1 and which constitute in this multi-layered network the fiber-like pivot arrays possibly described, in the average, as a 1D fiber bundle going through and somehow interconnecting the layers. This is one of the fully new results presented in this work, which shows how these pantograph-like deformation characteristics, called herein "geometrical descriptors" and which are linked to the physical connections (the pivots) between fiber layers, can be used to describe the corresponding behavior of the P-I composite structure where no pivots are physically accounted for. The other innovative development in this work is the proposed homogenization-based description for a two-phase bi-continuous P-I composite. In it, the reinforcing 3D fiber network needs be described in such a way that a realistic enough mGO can be calculated with the request of an easy follow up of its evolutions as induced by the high, pantographic-like, deformability properties that such a composite type is supposed to retain.

Section 2 first recalls from earlier author and co-worker works the Green-Fourier-Radon foundations of the used homogenization procedure for estimating effective properties of a two-phase composite and then presents the mGOs and the effective properties for the FPAs which serve to express those for the P-I fiber network to be embedded in a matrix. The Section 3, which contains the main new results of this work apart from the mGO approximate for FPAs of SQ-beams that appears in Section 2, presents the specific application to building the P-I fiber network we are interested in from a layered assemblage of FPAs with extension-evolving alternated misorientations, in order to calculate a representative mGO for it and then to estimate the current effective properties during deformation of the P-I composite. Two forms for these effective stiffness properties, obtained from two pretty close homogenization-based modeling procedures, are examined and compared, with special attention to well accounting in them for the properties of the through-layer interconnections. Once having shown that the two descriptions yield nearly same estimates for the examined structure under in-plane extension, the Section 4 reports some obtained results from that modeling scheme for the defined P-I composite and some performed comparisons with numerical simulations are presented and commented. At first are exemplified the effective (Young and shear) modulus evolutions with axial in-plane extension one arrives at for the P-I composite and the corresponding force-displacement curve resulting from the evolving effective axial Young modulus. These analytical results are then compared, for validation, with numerical simulations of the effective modulus evolutions during axial extension applied to a periodic structure that mimics at the best the P-I composite type of concern. At last, Section 4 compares the analytically obtained force-displacement curves, which are a priori free of torsion effects as built, with typical numerical curves from an in-plane extended 2D pantographic structure with appropriate end conditions for the overall behavior to be homogeneous. This allows to examining the matching possibilities between the respective models for these two structures (say the analytical modeling for two-phase P-I composite and the numerical one for a $2 \mathrm{D}$ pantographic bi-layer). It is first shown that pretty well adjusting a numerical curve on a given analytical one, which is characterized by its matrix to fibers stiffness ratio, is possible in adjusting the torsion modulus of the fiber inter-connecting pivots in the pantograph mechanical behavior description. Conversely examining the allowed ways to account for such pivot strengthening effects in the proposed analytical modeling where the fiber interconnections are not physically present, it is shown that: (i) for weak and moderate pivot strength effects, sufficient adjustment freedom is provided accounting for expectable (torsion-due) strengthening of the introduced throughlayer fiber bundle and/or possible (inclination-due) decrease of the layer inter-distances, with the fiber misorientation changes during extension; (ii) in the case of high pivot strength effects, a good analytical match can still be obtained in assuming a possible increase of the matrix stiffness with the extension-due fiber misorientation changes. This aptitude of the proposed P-I composite modeling, as shown here for an extension load, to analytically match the behavior of 2D pantographs provided the account of some indirect pivot strength effects, either through the fiber network or/and through the embedding matrix, also makes for certain applications the proposed P-I composite structure type a possible pantographequivalent that deserves further investigation. Section 5 concludes.

\section{Mean Green operator and effective properties of a planar alignment of fibers}

In many homogenization frameworks, the main role to estimate effective properties of heterogeneous structures of the reinforcedmatrix type is carried by the Eshelby tensors (Eshelby, 1957) which characterize how an infinite medium with given property $\boldsymbol{C}$ tensor constrains embedded domains $V$ in it which either have different properties or a different behavior from the matrix. Here on, the "properties" of concern will be only of the elastic type. In consequence, unless stated differently, any tensor or operator not explicated in component form is a rank-4 tensor. However, it is worth to specify that the entire recalled framework as well as the new developments in this work can be transcribed for dielectric-like properties or for so-called "generalized-elastic" ones, the latter allowing applications to coupled elastic-physical properties. In the former 
case, as explained in Franciosi (2005, 2013), this mostly amounts to substituting rank-4 tensors and operators with corresponding rank-2 ones while in the latter case it amounts to extend the rank4 tensor $(3,3,3,3)$ dimension to the appropriate one, according to the notation of Barnett and Lothe (1975).

The domains $V$ can be either inclusions which are shaperepresentative of the different embedded "inhomogeneities" or also domains whose shape represents a particular spatial distribution or organization symmetry of them in the matrix medium, as will be exemplified in the following. These widely used Eshelby tensors since then, which are still the topic of advanced research (Zhou et al., 2013; Traxl and Lackner, 2018; Eremeyev and Konopińska, 2019), were proved in the seminal work of Eshelby (1957) to be uniform in the interior of isolated (single) domains having an ellipsoidal shape. When a domain $V$ does not represent a single inclusion but a pattern of them, the interaction Eshelby tensors between all element pairs constituting the pattern are also needed to fully obtain the global (non uniform) tensor for $V$ (Berveiller et al., 1987). While the interior Eshelby tensor at $\boldsymbol{r}$ points of a $V$ domain (say $\boldsymbol{E}_{\boldsymbol{C}}^{V}(\boldsymbol{r}), \quad \boldsymbol{r} \in V$ ) is not easily at hand for a general $V$ shape even in the simplest cases of a matrix with isotropic properties, the interaction tensor between domain pairs is generally much harder to access, even with computational help. In order to estimate effective properties of reinforced matrices, it is often enough to consider, rather than the entire tensor field $\boldsymbol{E}_{\boldsymbol{C}}^{V}(\boldsymbol{r})$ over $V$, a mean tensor form over $V$, say $\overline{\boldsymbol{E}_{\boldsymbol{C}}^{V}}$, which can be computed in a simpler way: this mean global tensor comprises the appropriately weighted sum of all the mean interior tensors for the pattern elements and of all the mean pair interaction tensors between the elements. The product $\boldsymbol{E}_{\boldsymbol{C}}^{V}(\boldsymbol{r}): \boldsymbol{C}^{-1}$ between the Eshelby tensor and the compliance (inverse stiffness) tensor $\boldsymbol{C}^{-1}$ of the matrix in which $V$ is embedded defines the so-called modified Green operator integral, derived from the Green tensor, to be denoted $\boldsymbol{t}_{\boldsymbol{C}}^{V}(\boldsymbol{r})$ and named "GO" (Willis, 1971; Zeller and Dederich, 1973; Willis and Acton, 1976; Walpole, 1981; Kroner, 1958, 1990; Cheng et al., 1997; Pan and Tonon, 2000). If mean values over $V$ are to be considered, we have $\overline{\boldsymbol{E}_{\boldsymbol{C}}^{V}}: \boldsymbol{C}^{-1}=\overline{\boldsymbol{t}_{\boldsymbol{C}}^{V}}$ and when uniform in $V$, one will note $\boldsymbol{E}_{\boldsymbol{C}}^{V}(\boldsymbol{r})=\overline{\boldsymbol{E}_{\boldsymbol{c}}^{V}}=\boldsymbol{E}_{\boldsymbol{C}}^{V}$, (resp. $\left.\boldsymbol{t}_{\boldsymbol{c}}^{V}(\boldsymbol{r})=\overline{\boldsymbol{t}_{\boldsymbol{C}}^{V}}=\boldsymbol{t}_{\boldsymbol{c}}^{V}\right)$, $\forall \boldsymbol{r} \in V$ (in this latter case, $V$ can be specified to be necessarily ellipsoidal in writing $\left.V_{\text {ell }}\right)$. The knowledge of $\boldsymbol{t}_{\boldsymbol{C}}^{V}(\boldsymbol{r})$ is quite equivalent to the knowledge of $\boldsymbol{E}_{\boldsymbol{C}}^{V}(\boldsymbol{r})$ with several advantages of the former on the latter as positive definiteness and super symmetry in the case of elastic-like properties (Hill, 1952; 1965; Ponte Castaneda and Willis, 1995; Bornert et al., 1996; Buryachenko, 2001; Buryachenko and Brun, 2012). Some other advantages of the GO on the Eshelby tensor show up when making use of the Radon transform $(R T)$ method and inversion (IRT) formula to explicate it (Gel'fand et al. , 1966; Helgason, 1980; Natterer, 1986; Ramm and Katsevich, 1996), as its multiplicative dissociation into a geometric contribution and a material property one, which results in easier calculations as recalled next.

\subsection{Introduction of the Green operator and of the Radon transform and inverse formulae}

This recall is a synthesis of the RT-IRT presentation for application to inclusion problems from Franciosi and Lormand (2004), Franciosi (2005) and Franciosi and El Omri (2011), with adapted focus for the here addressed new inclusion pattern and structure type. We consider that infinite homogeneous elastic medium which is described by an elastic modulus tensor $\boldsymbol{C}$. We express the displacement field $\boldsymbol{u}(\boldsymbol{r})$ at point $\boldsymbol{r}$ due to a punctual force $\boldsymbol{f}\left(\boldsymbol{r}^{\prime}\right)$ applied to a different point $\boldsymbol{r}$ ' of the medium, with $\boldsymbol{u}=\left(u_{1}, u_{2}, u_{3}\right)$ (resp. $\boldsymbol{f}$ ), and $\boldsymbol{r}=\left(x_{1}, x_{2}, x_{3}\right)$ (resp. $\left.\boldsymbol{r}^{\prime}\right)$ as:

$u_{p}(\boldsymbol{r})=G_{p j}\left(\boldsymbol{r}-\boldsymbol{r}^{\prime}\right) f_{j}\left(\boldsymbol{r}^{\prime}\right)$ where $G_{p j}\left(\boldsymbol{r}-\boldsymbol{r}^{\prime}\right)$ is the Green strain tensor as defined in Hill (1952) and Kröner (1958). The medium being infinite, the Green tensor in Eq. (1) only depends on the distance $\boldsymbol{r}-\boldsymbol{r}^{\prime}$. Using Eq. (1) in the stress equilibrium relation $\sigma_{m n, n}(\boldsymbol{r})=\partial \sigma_{m n}(\boldsymbol{r}) / \partial x_{n}=\left(C_{m n p q}(\boldsymbol{r}) u_{p, q}(\boldsymbol{r})\right), n=0, \quad$ where $\boldsymbol{\sigma}(\boldsymbol{r})$ is the stress tensor, the property field $\boldsymbol{C}(\boldsymbol{r})$ is shared as $C_{\text {mnpq }}(\boldsymbol{r})=C_{m n p q}+\Delta C_{m n p q}(\boldsymbol{r})$, with $\boldsymbol{C}$ the reference medium property tensor. Treating the non uniform part $\left(\Delta C_{m n p q}(\boldsymbol{r}) u_{p, q}(\boldsymbol{r})\right), n$ as a punctual force field $f_{m}(\boldsymbol{r})=\delta\left(\boldsymbol{r}-\boldsymbol{r}^{\prime}\right) \delta_{m j} f_{j}\left(\boldsymbol{r}^{\prime}\right)$, with $\delta(\boldsymbol{r})$ the Dirac delta function and $\delta$ the Kronecker symbol, the twice differentiated Green tensor is defined from the relation:

$\left(C_{m n q q} G_{p j, q n}\left(\boldsymbol{r}-\boldsymbol{r}^{\prime}\right)+\delta\left(\boldsymbol{r}-\boldsymbol{r}^{\prime}\right) \delta_{m j}\right) f_{j}\left(\boldsymbol{r}^{\prime}\right)=0$

In Eq. (2) the double derivative at $\boldsymbol{r}$ of the Green tensor $G_{p j}\left(\boldsymbol{r}-\boldsymbol{r}^{\prime}\right)$ is named the modified Green operator, which reads (upon "super symmetrization" on index pairs (p,q),(j,n) and on $(\mathrm{p}, \mathrm{q})$ vs $(\mathrm{j}, \mathrm{n}))$ :

$\Gamma_{p q j n}\left(r-r^{\prime}\right)=-\left(\left.\frac{\partial^{2} G_{p j}\left(r-r^{\prime}\right)}{\partial x_{q} \partial x_{n}}\right|_{(p, q),(j, n)}\right)$

We next consider a domain $V$ into the infinite medium. Integrating the operator $\Gamma_{p q j n}\left(\boldsymbol{r}-\boldsymbol{r}^{\prime}\right)$ with regard to $\boldsymbol{r}^{\prime}$ over this domain, formally yields the modified Green operator integral at each interior or exterior point $\boldsymbol{r}$ to $V$, as (the reference to the infinite medium properties $\boldsymbol{C}$ is momentarily skipped):

$t_{p q j n}^{V}(\boldsymbol{r})=\int_{V} \Gamma_{p q j n}\left(\boldsymbol{r}-\boldsymbol{r}^{\prime}\right) d \boldsymbol{r}^{\prime}$.

It is this operator in Eq. (4) which is frequently called the "Green operator" for short that we here on note GO and mGO its mean value over $V$. An explicit calculus of $\boldsymbol{t}^{V}(\boldsymbol{r})$ over $V$ starts by Fourier transforming Eq. (2). With the wave vector $\boldsymbol{k}=\left(k_{1}, k_{2}, k_{3}\right)$ in the Fourier space having modulus $|\boldsymbol{k}|=k$ and unit vector $\boldsymbol{\omega}=\left(\omega_{1}, \omega_{2}, \omega_{3}\right)$ such that $\boldsymbol{k}=\left(k \omega_{1}, k \omega_{2}, k \omega_{3}\right)$, the Fourier transform ${ }^{3}$ of Eq. (2) is:

$C_{m n p q} \hat{G}_{p j}(\boldsymbol{k}) k_{q} k_{n}=\delta_{m j}$,

and introducing spherical coordinates such that $\omega_{1}=\sin \theta \cos \varphi$, $\omega_{2}=\sin \theta \sin \varphi, \omega_{3}=\cos \theta$ further yields:

$$
\begin{aligned}
C_{m n p q} \omega_{n} \omega_{q} k^{2} \hat{G}_{p j}(\boldsymbol{k}) & =M_{m p} k^{2} \hat{G}_{p j}(\boldsymbol{k})=\delta_{m j} \\
& =>k^{2} \hat{G}_{p j}(\boldsymbol{k})=M_{p j}^{-1}(\boldsymbol{\omega})
\end{aligned}
$$

Next replacing in Eq. (4) $\Gamma_{\text {pqin }}\left(\boldsymbol{r}-\boldsymbol{r}^{\prime}\right)$ by the inverse transform of its Fourier transform yields:

$t_{p q j n}^{V}(\boldsymbol{r})=\frac{1}{8 \pi^{3}} \int_{V}\left(\int\left\{M_{p j}^{-1}(\boldsymbol{\omega}) \omega_{q} \omega_{n}\right\}_{(p, q),(j, n)} e^{-i \boldsymbol{k} \cdot\left(\boldsymbol{r}-\boldsymbol{r}^{\prime}\right)} d \boldsymbol{k}\right) d \boldsymbol{r}^{\prime}$

Writing finally $d k=k^{2} d k \sin \theta d \theta d \varphi=k^{2} d k d \omega$, and with $\Omega$ the unit sphere in $R^{3}$, one obtains:

$t_{p q j n}^{V}(\boldsymbol{r})=\frac{1}{8 \pi^{3}} \int_{V}\left(\int_{\Omega} t_{p q j n}^{e}(\boldsymbol{\omega}) \int_{k=0}^{\infty} k^{2} e^{-i \boldsymbol{k} \cdot\left(\boldsymbol{r}-\boldsymbol{r}^{\prime}\right)} d k d \boldsymbol{\omega}\right) d \boldsymbol{r}^{\prime}$

where:

$t_{p q j n}^{e}(\boldsymbol{\omega})=M_{p j}^{-1}(\boldsymbol{\omega}) \omega_{q} \omega_{n}=\left(C_{a b c d} \omega_{b} \omega_{d}\right)_{p j}^{-1} \omega_{q} \omega_{n}$.

${ }^{3}$ Used definitions for the Fourier transform and inverse of $\boldsymbol{G}(\boldsymbol{r})$ and $\delta(\boldsymbol{r}) \quad$ are $\quad \hat{G}_{p j}(\boldsymbol{k})=\int G_{p j}(\boldsymbol{r}) \exp ^{i \boldsymbol{k} \cdot \boldsymbol{r}} d \boldsymbol{r} ; \hat{\delta}(\boldsymbol{k})=\int \delta(\boldsymbol{r}) \exp ^{i \boldsymbol{k} \cdot \boldsymbol{r}} d \boldsymbol{r}=1 ; \quad G_{p j}(\boldsymbol{r})=$ $(2 \pi)^{-3} \int \hat{G}_{p j}(\boldsymbol{k}) \exp ^{-i \boldsymbol{k} \cdot \boldsymbol{r}} d \boldsymbol{k} ; \delta(\boldsymbol{r})=(2 \pi)^{-3} \int \exp ^{-i \boldsymbol{k} \cdot \boldsymbol{r}} d \boldsymbol{k}$. 
Eq. (7b) can be simply rearranged by permuting the $V$ and $\Omega$ integrals and by introducing a shape (weight) function $\psi_{V}(\boldsymbol{\omega}, \boldsymbol{r})$ such as to finally write:

$t_{p q j n}^{V}(\boldsymbol{r})=\int_{\Omega} t_{p q j n}^{e}(\boldsymbol{\omega}) \psi_{V}(\boldsymbol{\omega}, \boldsymbol{r}) d \boldsymbol{\omega}$,

where:

$$
\begin{aligned}
\psi_{V}(\boldsymbol{\omega}, \boldsymbol{r}) & =\frac{1}{8 \pi^{3}} \xi_{V}(\boldsymbol{\omega}, \boldsymbol{r}), \quad \xi_{V}(\boldsymbol{\omega}, \boldsymbol{r}) \\
& =\int_{V}\left(\int_{k=0}^{\infty} k^{2} e^{-i k \boldsymbol{\omega} \cdot\left(\boldsymbol{r}-\boldsymbol{r}^{\prime}\right)} d k\right) d \boldsymbol{r}^{\prime}
\end{aligned}
$$

These calculations were presented with some more details in Franciosi and Lormand (2004) where it is shown that Eq. (8) is the IRT form of $t_{p q j n}^{V}(\boldsymbol{r})$. The first IRT powerfulness is to separate the concerned operator in two parts: (i) the $t_{p q j n}^{e}(\boldsymbol{\omega})$ elementary operators, which identify to the uniform GOs of laminates with normal $\boldsymbol{\omega}$, carry the $\boldsymbol{C}$ material property-orientation-dependent part (for each material property case, a Table of them can be calculated once and for all). Characteristic details are summarized in Appendix B which gives the non zero terms of $\boldsymbol{t}^{e}(\boldsymbol{\omega})$ for the case of isotropic elasticity; (ii) the shape function $\psi_{V}(\boldsymbol{\omega}, \boldsymbol{r})$ of a specific inclusion or pattern $V$ is only defined by the geometry of $V$ and is valid regardless of the properties of the embedding matrix, whether them be isotropic or not and whether them be elastic or else. The function $\psi_{V}(\boldsymbol{\omega}, \boldsymbol{r})$ is the IRT of the characteristic (indicator) function of $V$, which equals 1 (resp. 0 ) at all points $\boldsymbol{r}$ interior (resp. exterior) to $V$. However, although both parts of the GO in Eq. (8) are at analytical hand for quite many cases, the resolution of these integrals (for the different pqjn operator terms) can remain quite complicated especially for general property anisotropy. The Appendix B also shows the form of the individual double $(\theta$, $\varphi)$ integrals over the unit sphere which need be calculated from Eq. (8) to obtain the terms of the mGO $\overline{\boldsymbol{t}^{V}}$ for some $V$ domain of which the mean shape function $\overline{\psi_{V}(\boldsymbol{\omega})}$ is available with indications for the calculation of the FPA mGOs here of use. Now on is briefly recalled the calculation route of the $\xi_{V}(\boldsymbol{\omega}, \boldsymbol{r})$ function from the RT-IRT method in order to obtain that shape function and then its mean value $\overline{\psi_{V}(\boldsymbol{\omega})}$ over $V$ when $V$ is the fiber network organization to be embedded in a matrix in order to realize the new P-I composite type of concern in this work.

Let's consider a $(\boldsymbol{x}, \boldsymbol{y}, \boldsymbol{z})$ frame with $\mathrm{Oz} / / \boldsymbol{k} / / \boldsymbol{\omega}$, such that $k \cdot$ $r=k \boldsymbol{\omega} \cdot r=k z$. This allows to rewrite $\xi_{V}(\boldsymbol{\omega}, \boldsymbol{r})$ in Eq. (9a) under a form which identifies the $1 \mathrm{D} k$-integral with the inverse Fourier transform of the second $z$-derivative of the delta function, say:

$$
\begin{aligned}
\xi_{V}(\boldsymbol{\omega}, r) & =-\frac{1}{2} \int_{V}\left(\int_{-\infty}^{+\infty}(i t)^{2} \exp ^{-i t\left(z-z^{\prime}\right)} d t\right) d r^{\prime} \\
& =-\pi \int_{V} \delta^{\prime \prime}\left(z-z^{\prime}, \omega\right) d r^{\prime},
\end{aligned}
$$

from which the function $\psi_{V}(\boldsymbol{\omega}, \boldsymbol{r})$ can be written, using for each direction $\boldsymbol{\omega}$ the decomposition $d r^{\prime}=d s_{V}\left(z^{\prime}, \boldsymbol{\omega}\right) d z^{\prime}$, as:

$$
\begin{aligned}
\psi_{V}(\boldsymbol{\omega}, \boldsymbol{r}) & =-\int_{V} \frac{\delta^{\prime \prime}\left(\boldsymbol{\omega} \cdot\left(\boldsymbol{r}-\boldsymbol{r}^{\prime}\right)\right)}{8 \pi^{2}} d r^{\prime} \\
& =-\int_{z^{\prime}=D_{V}^{-}(\boldsymbol{\omega})}^{z^{\prime}=D_{V}^{+}(\boldsymbol{\omega})}\left(\int_{s_{V}\left(z^{\prime}, \omega\right)} d s_{V}\left(z^{\prime}, \boldsymbol{\omega}\right)\right) \frac{\delta^{\prime \prime}\left(z-z^{\prime}, \boldsymbol{\omega}\right)}{8 \pi^{2}} d z^{\prime} \\
& =-\frac{\tilde{s}_{V}^{\prime \prime}(z, \boldsymbol{\omega})}{8 \pi^{2}}
\end{aligned}
$$

In Eq. (10), the integral $\int_{s_{V}\left(z^{\prime}, \omega\right)} d s_{V}\left(z^{\prime}, \boldsymbol{\omega}\right)=s_{V}(z, \boldsymbol{\omega})$ is the planar section area of $V$ when cut by the plane of equation $z=\omega . r$ that passes through the point $\boldsymbol{r}$ and is normal to the direction $\boldsymbol{\omega}$. Then, still using $\boldsymbol{\omega}=(\sin \theta \cos \phi, \sin \theta \sin \phi, \cos \theta)$ for the vectors over the unit sphere $\Omega, \tilde{s}_{V}^{\prime \prime}(z, \boldsymbol{\omega})=\frac{\partial^{2}}{\partial z^{2}} s_{V}(z, \boldsymbol{\omega})$ is the second $z$-derivative of $s_{V}(z, \omega)$, to be understood as indicated by the tilde, as a regularized form of it if necessary, in the sense of distributions (Gelf'and et al., 1966; Franciosi, 2010; 2018). This second $z$-derivative of $s_{V}(z, \omega)$ results from the definition of the p-derivatives $\delta^{(p)}(x)$ of the delta function which fulfill $\int f(x) \delta^{(p)}\left(x-x_{0}\right) d x=(-1)^{p} f^{(p)}\left(x_{0}\right)$ for any suitable (continuous, compactly supported, $\mathrm{p}$-differentiable, ...) function $f(x)$ in some integration interval over a neighborhood of point $x_{0}$. Next setting $d r=d s_{V}(z, \boldsymbol{\omega}) d z$ as having set $d r^{\prime}=d s_{V}\left(z^{\prime}, \boldsymbol{\omega}\right) d z^{\prime}$ in Eq. (10), the mean value of this $V$ shape function reads, $\forall \omega \in \Omega$ and with $\mathrm{v}$ the volume of $\mathrm{V}$ :

$$
\begin{aligned}
\overline{\psi_{V}(\boldsymbol{\omega})} & =-\frac{1}{8 \pi^{2} v} \int_{D_{V}^{-}(\boldsymbol{\omega})}^{D_{V}^{+}(\boldsymbol{\omega})} \tilde{s}_{V}^{\prime \prime}(z, \boldsymbol{\omega}) s_{V}(z, \boldsymbol{\omega}) d z \\
& =\frac{1}{8 \pi^{2} v} \int_{D_{V}^{-}(\boldsymbol{\omega})}^{D_{V}^{+}(\boldsymbol{\omega})}\left(\tilde{s}_{V}^{\prime}(z, \boldsymbol{\omega})\right)^{2} d z .
\end{aligned}
$$

The interval $\left[D_{V}^{-}(\boldsymbol{\omega}), D_{V}^{+}(\boldsymbol{\omega})\right]=2 D_{V}(\boldsymbol{\omega})$ corresponds to the breadth of $V$ in the $\omega$ direction (i.e. the distance between the two opposite tangent planes to $V$, of $\omega$-normal), which characterizes the support function of $V$ when strictly convex $\left(D_{V}(\boldsymbol{\omega})=\max _{\boldsymbol{r} \in V}(\boldsymbol{\omega} . \boldsymbol{r})\right)$, or more generally, in particular when $V$ is a pattern of inclusions, of the convex hull of $V$. In the limit of infinite $V$ domains or patterns, the breadth of $V$ becomes infinite.

This framework holds as well for inclusion patterns since it is established (Berveiller et al., 1987) that similar calculation can be applied to the case of a pair of inclusions $V=V_{1} \cup V_{2}=, V_{1} \cap V_{2}=\Phi$ with $\Phi$ the empty set), and from an inclusion pair to any inclusion number $n$ in a pattern $V=\bigcup_{i=1}^{n} V_{i}$ up to infinite $n$-sets which can be seen as multiply connected inclusions $V$ (Franciosi, 2010, 2019; Franciosi et al., 2015,2019). At any interior point $\boldsymbol{r}$ of such a $V$ pattern of $n$ (possibly infinite) $V i$ inclusion number, the shape function and the related GO comprises the interior term from $V i$ when $\boldsymbol{r}$ is interior to $V \mathrm{i}$ and all the exterior contributions from the other $V \mathrm{j}$ domains $(j \neq i)$. At exterior points to $V$ both the shape function and the GO assemble the $n$ exterior contributions from all the $V \mathrm{j}$ domains which constitute $V$. As far as the interior $\mathrm{mGO}$ of a general $V$ pattern is of concern here, the mean shape function for an (up to infinite) number of inclusions then reads from Eq. (10):

$$
\overline{\psi_{V}(\boldsymbol{\omega})}=\frac{1}{8 \pi^{2} v} \int_{D_{V}^{-}(\boldsymbol{\omega})}^{D_{V}^{+}(\boldsymbol{\omega})}\left(\sum_{i=1}^{n} \tilde{s}_{V i}^{\prime}(z, \boldsymbol{\omega})\right)\left(\sum_{j=1}^{n} \tilde{s}_{V j}^{\prime}(z, \boldsymbol{\omega})\right) d z,
$$

with $v=\sum_{i=1}^{n} v_{i}$

$$
\overline{\psi_{V}(\boldsymbol{\omega})}=\sum_{i=1}^{n} \frac{v_{i}}{v} \overline{\psi_{V i}(\boldsymbol{\omega})}+\sum_{i=1}^{n} \sum_{j=i+1}^{n}\left(\frac{v_{i}+v_{j}}{v}\right) \overline{\psi_{V i, V j}(\boldsymbol{\omega})} .
$$

From comparing Eq. (12a) with Eq. (11), it is immediate to see that:

$$
\begin{aligned}
\overline{\psi_{V i}(\boldsymbol{\omega})} & =\int_{D_{V i}^{-}(\boldsymbol{\omega})}^{D_{V i}^{+}(\boldsymbol{\omega})} \frac{\left(\tilde{s}_{V i}^{\prime}(z, \boldsymbol{\omega})\right)^{2}}{8 \pi^{2} v_{i}} d z \\
\overline{\psi_{V i, V j}(\boldsymbol{\omega})} & =\int_{\sup \left(D_{V i}^{-}(\boldsymbol{\omega}), D_{V j}^{-}(\boldsymbol{\omega})\right)}^{\inf \left(D_{V i}^{+}(\boldsymbol{\omega}), D_{V j}^{+}(\boldsymbol{\omega})\right)} \frac{\tilde{s}_{V i}^{\prime}(z, \boldsymbol{\omega}) \tilde{S}_{V j}^{\prime}(z, \boldsymbol{\omega})}{8 \pi^{2}\left(v_{i}+v_{j}\right)} d z .
\end{aligned}
$$

The pattern mGO follows as:

$\overline{\boldsymbol{t}^{V}}=\sum_{i=1}^{n} \frac{v_{i}}{v} \overline{\boldsymbol{t}^{V i}}+\sum_{i=1}^{n} \sum_{j=i+1}^{n}\left(\frac{v_{i}+v_{j}}{v}\right) \overline{\boldsymbol{t}^{V i, V j}}$, with $v=\sum_{i=1}^{n} v_{i}$.

Infinite series in Eq. (12) formally provide the mean shape function and the related mGO for infinite inclusion patterns. The first (simple sum) term corresponds to the interior parts from the individual pattern elements and reduces to a single term when all 
Table 3

Non zero interior mGO terms for $x 2$-oriented generic fibers, C-fibers, SQ-beams and $x 2$ - $x 1$ laminates in isotropic media $(B=1 / \mu, A=-B / 2(1-v)), S^{(2)}=1-C^{(2)},\left(S^{(2)} C^{(2)}\right)=C^{(2)}-C^{(4)}, S^{(4)}=1-2 C^{(2)}+$ $C^{(4)}$.

\begin{tabular}{|c|c|c|c|c|c|c|}
\hline & 1111 & 1133 & 3333 & 2323 & 1313 & 1212 \\
\hline$\overline{\boldsymbol{t}_{\boldsymbol{C}^{M}}^{\text {Genfiber } \boldsymbol{x} 2}}$ & $A S^{(4)}+B S^{(2)}$ & $A\left(S^{(2)} C^{(2)}\right)$ & $A C^{(4)}+B C^{(2)}$ & $B C^{(2)} / 4$ & $A\left(S^{(2)} C^{(2)}\right)+B / 4$ & $B S^{(2)} / 4$ \\
\hline$\overline{\boldsymbol{t}_{\mathrm{C}^{M}}^{C-\text { fiber }, \boldsymbol{x} 2}}$ & $\frac{3 A+4 B}{8}$ & $\frac{A}{8}$ & $\frac{3 A+4 B}{8}$ & $\frac{B}{8}$ & $\frac{A+2 B}{8}$ & $\frac{B}{8}$ \\
\hline $\begin{array}{l}\boldsymbol{t}_{C M}^{S Q-B e a m, \boldsymbol{x} 2} \\
\boldsymbol{t}_{C M}^{\operatorname{Lam}, \boldsymbol{x} 2-\boldsymbol{x} 1}\end{array}$ & $\frac{(6-\pi) A+4 B}{8}$ & $\frac{(\pi-2) A}{8}$ & $\frac{\frac{(6-\pi) A+4 B}{8}}{A+B}$ & $\begin{array}{l}\frac{D}{8} \\
\frac{B}{8} / 4\end{array}$ & $\frac{(\pi-2) A+2 B}{8}$ & $\begin{array}{l}0 \\
B \\
0\end{array}$ \\
\hline
\end{tabular}

elements are identical. ${ }^{4}$ The second (double sum) term represents the global interaction part in the pattern mGO. Only the interaction term depends on the element inter-distances.

As far as the matrix phase is considered to have isotropic elastic-like properties, all the non-vanishing components of the $\mathrm{mGO}$ for any inclusion pattern only depend on and can be expressed from a limited set of independent functions which depend on the relative positions of the inclusions in the pattern, through the interaction terms in the shape function of Eq. (12b) and on the pattern orientation in the chosen $(x 1, x 2, x 3)$ frame. In the FPA cases, only two independent functions are enough, as $C 2$ and $C 4$ say, ${ }^{5}$ the way these two functions appear in the double integrals in Eq. (8) which explicate the mGO terms for any pattern $V$ of concern is recalled in Appendix B, with some details recalled for obtaining the FPA mGO two functions. Thanks to these simplifying characteristics, a first case of infinite pattern (say coaxial alignments of non necessarily all size-identical oblate spheroids including spheres) was explicated in Franciosi (2010) for isotropic matrix properties, and prolate spheroids being treatable identically. The case of aligned coaxial finite cylinders with same radius was next treated in Franciosi (2014) and Franciosi et al. (2015) still for isotropic (elastic-like or dielectric-like) matrix properties. In the case of inclusion patterns made of parallel equally distant fibers in a plane, these two functions can be written as $C 2(x)$ and $C 4(x)$, with " $x$ " standing for a normalized characteristic of the element inter-distance in the FPAs, to be specialized for each fiber type. According to Eq. (12), these two functions share into an $x$ independent part $C^{(2)}, C^{(4)}$ which corresponds to the interior fiber mGO term and into a global interaction part, the rest of it, between all the pattern element pairs, $C_{2}(x), C_{4}(x)$. This interaction part is the $x$-dependent part of the mGO, such that $C 2 l(x)=C^{(2 l)}+C_{2 l}(x)$, $l=1,2$.

Now on, the reported work specializes to 3D structures made from piling FPAs of $n$ parallel fibers of same cross section shape and size and equally inter-distant, with $n$ being large enough to be taken as infinite. The mGOs for such FPAs in isotropic elasticlike matrices were previously solved in exact analytical form in Franciosi (2018) for beams with rectangular cross sections and for cylinders in Franciosi et al. (2019), the related effective properties for both FPA types being also explicated. These calculations being lengthy, they have deserved to be published in preliminary works. Unnecessary details here must be found in the given references. The Appendix B simply explains the few essential steps. The exact mGO solution for cylindrical fibers of circular cross section (to be denoted C-fibers) are recalled for comparison with an accurately approximate analytical mGO form for infinitely many parallel beams of same square cross section (SQ-beams), which is newly introduced for being here used in easier way than the exact one (fiber cross sections in pantographs are closer to square than to

4 They only need be all congruent what allows them to possibly have different sizes.

${ }^{5}$ Isotropic dielectric-like properties only call for a single, for example the $C 2(x)$, function. circular). The interior parts of the $C 2(x)$ and $C 4(x)$ functions for the SQ-beam case read simply $C_{S Q B}^{(2)}=1 / 2$ and $C_{S Q B}^{(4)}=(6-\pi) / 8$. The $C_{S Q B}^{(2)}$ interior function equals the C-fiber one, $C_{C F i b}^{(2)}=1 / 2$ and the $C_{S Q B}^{(4)}$ one does not differ much from $C_{C F i b}^{(4)}=3 / 8$. The non zero components of the interior mGO of $\boldsymbol{x} 2$-oriented infinite fibers resulting from these two functions are reported in Table 3 in generic form and for the C-fibers and SQ beams here of concern. The uniform interior GO of laminates with $\boldsymbol{x} 3$ normal is also recalled.

\subsection{Planar alignments of C-fibers and of SQ-beams and their interaction mGOs}

Applying Eq. (12c) to a nFPA of C-fibers (cylinders of radius $R$ and of axis inter-distance $2 \mathrm{~L}$, denoted $n c y l$ ) embedded in a medium of isotropic elasticity tensor $\boldsymbol{C}$ yields the elastic $\mathrm{mGO}$ form explicated in Franciosi et al. (2019) for $\boldsymbol{x} 2$-oriented fibers and FPA planes of normal $\boldsymbol{x} 1$ :

$$
\begin{aligned}
\overline{\boldsymbol{t}_{\rho_{0}}^{n c y l}} & =\frac{1}{n}\left(n \boldsymbol{t}^{c y l}+2 \sum_{i=1}^{n-1}(n-i) \overline{\boldsymbol{t}_{\rho_{i}}^{c y l-c y l}}\right) \\
& =\boldsymbol{t}^{c y l}+2 \sum_{i=1}^{n-1} \frac{(n-i)}{n}\left(\frac{\boldsymbol{v}_{0}}{i^{2}} \rho_{0}^{2}+\frac{\boldsymbol{w}_{0}}{i^{4}} \rho_{0}^{4}\right) \\
& =\boldsymbol{t}^{c y l}+2\left(\sigma_{2}^{(n)} \boldsymbol{v}_{0} \rho_{0}^{2}+\sigma_{4}^{(n)} \boldsymbol{w}_{0} \rho_{0}^{4}\right) \\
& =\boldsymbol{t}^{c y l}+\left(\boldsymbol{v}_{0}^{(n)} \rho_{0}^{2}+\boldsymbol{w}_{0}^{(n)} \rho_{0}^{4}\right),
\end{aligned}
$$

with $\rho_{0}=R / L \leq 1$ being the $x$ parameter in the functions $C_{2 l}(x)$, $l=1,2$.

The interior part of this mGO is the $\boldsymbol{t}^{c y l}$ uniform GO of an infinite cylinder and the interaction $\mathrm{mGO}$ for any $n$ element number is a two-terms polynomial in $\rho_{0}^{2}$ and $\rho_{0}^{4}$, the coefficients of which are a series term time a tensor, denoted $2 \sigma_{2}^{(n)} \boldsymbol{v}_{0}=\boldsymbol{v}_{0}^{(n)}$ and $2 \sigma_{4}^{(n)} \boldsymbol{w}_{0}=\boldsymbol{w}_{0}^{(n)}$.

The two $\boldsymbol{v}_{0}=\boldsymbol{v}_{0}^{(2)}$ and $\boldsymbol{w}_{0}=\boldsymbol{w}_{0}^{(2)}$ tensors are defined from the C-fiber pair interaction $\left(n=2, i=1,2 \sigma_{2}^{(2)}=2 \sigma_{4}^{(2)}=1\right.$ and $\left.\overline{\boldsymbol{t}_{\rho_{0}}^{2 c y l}}=\boldsymbol{t}^{c y l}+\left(\boldsymbol{v}_{0} \rho_{0}^{2}+\boldsymbol{w}_{0} \rho_{0}^{4}\right)\right)$ and they represent the non zero terms of the pair interaction mGO between two parallel cylinders. For $n \rightarrow \infty$, the limit for the infinite series, $\Sigma(q)=\lim _{n \rightarrow \infty} \sigma_{q}^{(n)}=$ $\lim _{n \rightarrow \infty} \sum_{i=1}^{n-1} \frac{(n-i)}{n}\left(\frac{1}{i q}\right)$, is finite and corresponds to the Riemann Zeta function $Z(q)=\lim _{n \rightarrow \infty} \sum_{i=1}^{n-1}\left(\frac{1}{i q}\right), \forall q>1$. Thus, at the limit of an infinite C-fiber FPA (denoted $\infty c y l$ ) one obtains with $Z(2)=\pi^{2} / 6$, $Z(4)=\pi^{4} / 90$, the $\mathrm{mGO}$ form:

$$
\begin{aligned}
\overline{\boldsymbol{t}_{\rho_{0}}^{\infty c y l}} & =\boldsymbol{t}^{c y l}+\left(\boldsymbol{v}_{0}^{(\infty)} \rho_{0}^{2}+\boldsymbol{w}_{0}^{(\infty)} \rho_{0}^{4}\right) \\
& =\boldsymbol{t}^{c y l}+2\left(Z(2) \boldsymbol{v}_{0} \rho_{0}^{2}+Z(4) \boldsymbol{w}_{0} \rho_{0}^{4}\right),
\end{aligned}
$$

with the particular (not always the maximal) values when all the cylinders are at contact $\left(\rho_{0}=1\right)$ :

$\overline{\boldsymbol{t}_{\rho_{0}=1}^{\infty c y l}}=\boldsymbol{t}^{c y l}+2\left(Z(2) \boldsymbol{v}_{0}+Z(4) \boldsymbol{w}_{0}\right)$. 

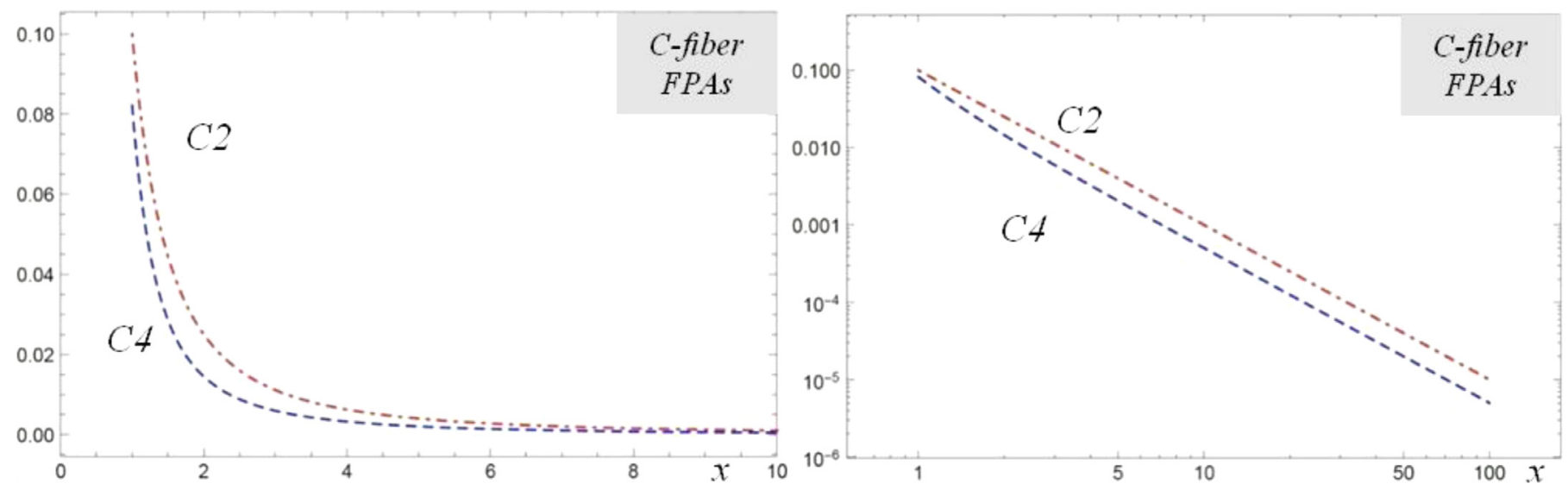

Fig. 6. The (left) $C_{2}(x)$ (red) and $C_{4}(x)$ (blue) mGO interaction function parts of C- fiber FPAs; (right) $\log / \log$ plot.

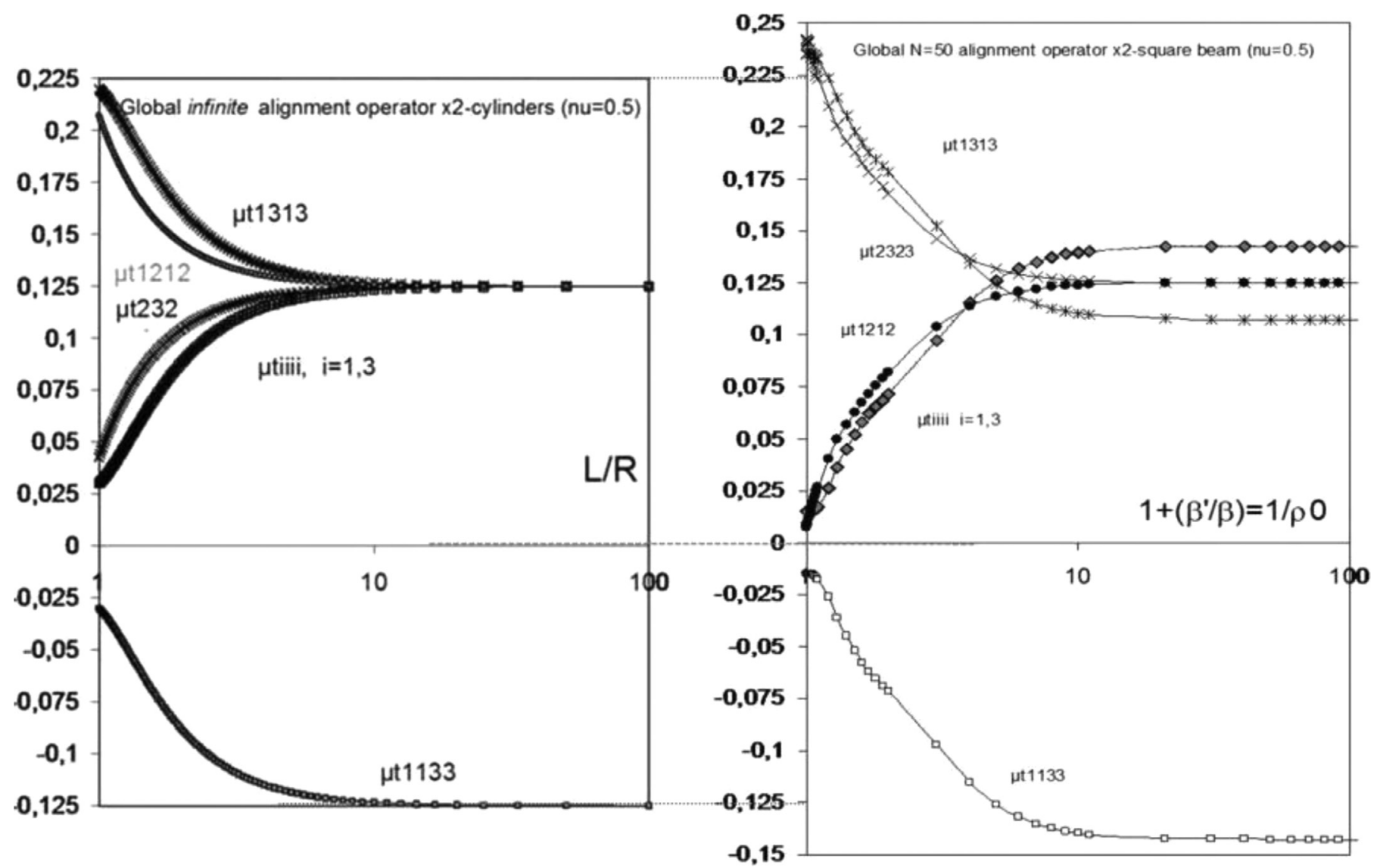

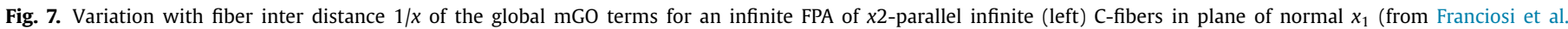
2019) and of (right) SQ-beams of square cross section in plane of normal $x_{3}$ (indices 1 and 3 are to be inverted) (from Franciosi, 2018).

More synthetically, the functions $C_{2}(x)$ and $C_{4}(x)$ for the interaction part of the $\mathrm{mGO}$ for infinite FPAs of normal $\boldsymbol{x} 1$ and of $\boldsymbol{x} 2$ oriented C-fibers that yield Eq. (13) can be exactly written, with the $Q_{i}, S_{i}$ coefficient values given in Table 4 , as the polynomial form $\left(x=\rho_{0}\right)$ :

$C_{2 l}^{\infty c y l}(x)=Q_{2 l} x^{2}+S_{2 l} x^{4}, \quad l=1,2$.

The Fig. 6, shows these $C_{2}(x)$ and $C_{4}(x)$ functions for the interaction part of the mGO for FPAs of C-fibers, according to Eq. (14). The variations of the non zero terms of the $\mathrm{mGO}$ with the C-fiber relative (normalized) inter distance $(1 / x=L / \mathrm{R})$ according to Eq. (13) are plotted in Fig. 7 left from Franciosi et al. (2019) for a Poisson ratio of 0.5 . When the fiber inter-distance increases, the global mGO re-
Table 4

Coefficients of $C_{2 l}(x)$ functions, $l=1,2$,

for C-fiber infinite FPAs in Eq. (14).

\begin{tabular}{lll}
\hline & $C_{2}(x)$ & $C_{4}(x)$ \\
\hline$Q$ & -0.1 & -0.05 \\
$S$ & 0. & -0.0321 \\
\hline
\end{tabular}

duces to its interior part. At fiber contact, the mGO interaction part is optimal, but terms can either be positive or negative (the global $\mathrm{mGO}$ is always positive definite). 

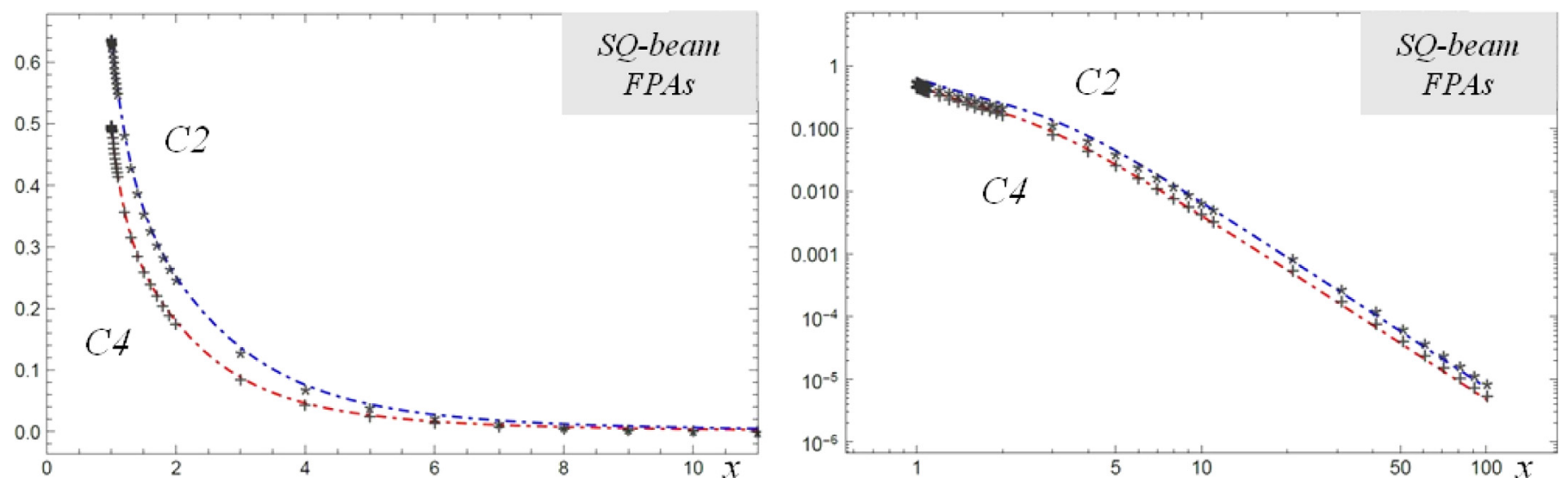

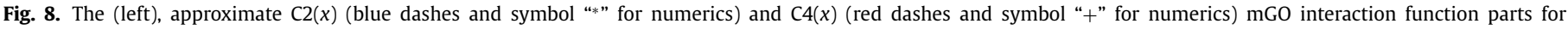
squared beam FPAs; (right) $\log / \log$ plot.

Table 5

coefficients of $C_{2 l}(x)$ functions, $l=1,2$, for SQ-beam infinite FPAs in Eq. (15a).

\begin{tabular}{lll}
\hline & $C_{2}(x)$ & $C_{4}(x)$ \\
\hline$R$ & 0.786 & 0.162 \\
$Q$ & -1.330 & -0.376 \\
$S$ & 1.043 & 0.656 \\
$\alpha$ & 0.162 & 0.049 \\
\hline
\end{tabular}

The Fig. 7 right reports from Franciosi (2018), the corresponding global mGO non zero term variations for nearly infinite ${ }^{6}$ nFPAs of SQ-beams with the normalized inter-distance $(1 / x) .^{7}$ Here $\rho_{0}=$ $x \leq 1$ is defined as $\beta /(\beta+\beta$ ') with $\beta$ the square beam half side and $\beta^{\prime}$ the half inter-space between beam pairs (equal zero at contact) the axis inter distance being then $2\left(\beta+\beta^{\prime}\right)$. As for the C-fiber alignments, this Figure shows that the influence zone for all the pair interactions between SQ-beams in an infinite FPA is also ten to twenty times the beam width, depending on the wished precision. These variations of the mGO interaction part in the global mGO for SQ-beams were obtained from using the exact although complicated solution reported in the cited reference, not to be repeated here. From a purely graphical analysis of the numerical plots reported in Fig. 8 (from that exact solution for the mGO interaction part of infinite SQ-beam in FPAs) a simplified form of easier use is shown to write from two functions $C_{2 l}(x)(l=1,2)$ which can be expressed as:

$C_{2 l}^{\infty \text { Beam }}(x)=x^{2}\left(R_{2 l} x^{-2}+Q_{2 l} x^{-1}+S_{2 l}\right) \frac{1}{1+\alpha_{2 l}\left(x^{-3}-1\right)}$,

where the involved coefficients are reported in Table 5.

For the reason that in pantographs the fiber inter distance is generally at most of the order of ten times the fiber dimension, it is possible to consider here a further shortened form of Eq. (15a), (without the $\alpha$ mostly long-range adjustment), say the rank-2 polynomial form $(l=1,2)$ :

$C_{2 l}^{\infty \text { Beam }}(x) \simeq x^{2}\left(R_{2 l} x^{-2}+Q_{2 l} x^{-1}+S_{2 l}\right)=R_{2 l}+Q_{2 l} x+S_{2 l} x^{2}$

The Table 6 reports the generic form (say holding for any common cross section shape given to the aligned fibers) of the inter-

\footnotetext{
${ }^{6}$ The (finite) limit of this infinite series having no analytical closed form for SQ beams, the series is truncated at $n=50$.

7 Indices 1 and 3 are inverted since the alignment is of normal $x 1$ for C-fibers and of normal $x 3$ for SQ-beams.
}

action $^{8} \mathrm{mGO}$ for an infinite FPA of $\boldsymbol{x} 2$-oriented fibers in the plane of normal $\boldsymbol{x} 3$, denoted $\overline{\boldsymbol{t}_{\boldsymbol{C}^{M}}^{\text {int( } \infty F P A) 0}}$. This Table also specifies the particular mGOs for FPAs of C-fibers and of SQ-beams (the $\boldsymbol{x} 2$ axis is now on always taken to correspond to the $\theta=0$ angle for FPA orientations as defined in Figs. 1-3 and 5, not to be confused with the $\theta$ angle of the spherical $(\theta, \phi)$ coordinates which define the directions $\omega$ on the unit sphere $\Omega$ and the shape function terms.

Hence, the corresponding global mGO $\overline{\boldsymbol{t}_{\mathrm{CM}}^{\infty F P A(0)}}$ for an infinite (C-fiber or SQ-beam) FPA is the sum of the interaction part $\overline{\boldsymbol{t}_{\boldsymbol{C}^{M}}^{\text {int( } \infty F P A) 0}}$ from Table 6 and of the corresponding interior one $\overline{\boldsymbol{t}_{\boldsymbol{C}^{M}}^{\text {Genfiber, } \boldsymbol{x} 2}}$ from Table 3, both specializing as explicated for C-fibers or SQ-beams.

\subsection{Effective properties of the planar alignment of C-fibers and of SQ-beams}

As far as one considers a homogeneous medium embedding one (or more) phase(s) under the form of inclusions or inclusion patterns, in random manner, the use of the mean field twopoint statistics approximation from Ponte Castaneda and Willis (1995) allows to estimating effective properties in accounting for each phase volume fraction, properties and representative domain shape as the well established (Hashin-Shtrikman, 1963) estimate of which it is a generalization that additionally accounts for some global anisotropy of the spatial distribution symmetry of these phases. This here on called "PCW estimate" for $n$ included phases in matrix with properties $\boldsymbol{C}^{M}$, that we do not reintroduce, sending the readers to the PCW reference, was successfully applied for example in Franciosi (2013), Franciosi and Charles (2016b) and Franciosi et al. (2019) to various new inclusion and pattern reinforcements in the same spirit as in the present work, considering elastic properties or generalized elastic (say magneto-electroelastic) ones. For the following, it is enough to recall that it takes the generic form for $n$ different phases in a $(n++1)$ th matrix one:

$\boldsymbol{C}_{e f f P C W}^{V j / S D i s t}=\boldsymbol{C}^{M}-\left(\left(\sum_{j=1}^{n}\left(f_{V j}\left(\left(\boldsymbol{C}^{M}-\boldsymbol{C}^{V j}\right)^{-1}-\overline{\boldsymbol{t}_{\boldsymbol{C}^{M}}^{V j}}\right)\right)^{-1}\right)^{-1}+\boldsymbol{t}_{\boldsymbol{C}^{M}}^{S D i s t}\right)^{-1}$,

${ }^{8}$ The difference with the generic form of the interior mGO in Table 1 comes from the fact that the mean shape function integral over the unit sphere is always zero for the interaction part and unity for the interior part. 


\begin{tabular}{lllllll}
\hline & 1111 & 1133 & 3333 & 2323 & 1313 & 1212 \\
\hline$\overline{\boldsymbol{t}^{\mathrm{int}}(\infty F P A) 0}$ & $\mathrm{~A}\left(C_{4}-2 C_{2}\right)-\mathrm{BC} C_{2}$ & $\mathrm{~A}\left(C_{2}-C_{4}\right)$ & $\mathrm{AC}_{4}+B C_{2}$ & $C_{2} \mathrm{~B} / 4$ & $\mathrm{~A}\left(C_{2}-C_{4}\right)$ & $-C_{2} \mathrm{~B} / 4$ \\
\hline
\end{tabular}

where $\overline{\boldsymbol{t}_{\boldsymbol{C}^{M}}^{V j}}$ is formally the mGO of some representative domain $\mathrm{Vj}$ for phase $j$ having $\boldsymbol{C}^{V j}$ properties and $f_{V j}$ volume fraction, and $\boldsymbol{t}_{\boldsymbol{C}^{M}}^{S D i s t}$ is the GO representing some spatial distribution for all these $V j$ domains embedded in the matrix. This spatial distribution needing be formally ellipsoidal to rigorously obtain Eq. (16a) according to the cited PCW modeling, this $\boldsymbol{t}_{\boldsymbol{C}^{M}}^{\text {SDist }}$ operator is a uniform GO. ${ }^{9}$ This distribution symmetry operator can also be seen as the GO of the representative (ellipsoidal) elementary matrix volume $V_{M}$ containing the representative volume elements of the embedded phases. When the domains $V j$ are single inclusions of phase $\mathrm{j}$ (all in dilute concentrations), the PC-W estimate statistically accounts for a part of their interactions through their spatial distribution. But patterns at non dilute concentrations cannot be single phased what makes the general situation difficult to handle. The case of a single non dilute phase $(\mathrm{j})$ is simpler: the larger is the chosen representative pattern $V j$, the more the pair interactions can be accounted for precisely at the RVE scale, that is within the pattern mGO. And as far as the considered patterns of inclusions of the same $j$ phase are finite sets, the estimate from Eq. (16a) still additionally accounts for the pair interactions between any two patterns through their spatial distribution. In consequence, interactions between pairs of infinite patterns become a marginal extra contribution to effective property estimates when the element interactions in the pattern are accounted for in the pattern mGO. Thus, Eq. (16a) will be enough in the following with using infinite patterns to represent a single embedded phase. The two-phase form of Eq. (16a) simplifies to:

$$
\boldsymbol{C}_{e f f P C W}^{V / S D i s t}=\boldsymbol{C}^{M}-f_{V}\left(\left(\left(\boldsymbol{C}^{M}-\boldsymbol{C}^{V}\right)^{-1}-\overline{\boldsymbol{t}_{\mathbf{C}^{M}}^{V}}\right)+f_{V} \boldsymbol{t}_{\boldsymbol{C}^{M}}^{S D i s t}\right)^{-1} .
$$

In Eq. (16b), the single included phase has a representative inclusion pattern $V$ with $\overline{\boldsymbol{t}_{\boldsymbol{C}^{M}}^{V}}$ being its mGO, a volume fraction $f_{V}=1-f_{M}$ and elastic properties $\boldsymbol{C}^{V}$, and these patterns are spatially distributed according to some (ellipsoidal) symmetry represented by the $\boldsymbol{t}_{\boldsymbol{c}^{M}}^{S \text { Dist }}$ uniform GO. Assuming statistical homogeneity for the individual elements, regardless of the pattern details, $f_{V}$ must also be the inclusion concentration in the pattern, say the volume fraction of $V$ in its elementary matrix volume $V_{M}$ (a difference would represent some inclusion clustering in the patterns, the statistical homogeneity assumption applying at the pattern distribution scale). When such a composite is deformed, both the characteristic embedded inclusion pattern and their spatial distribution evolve. In the particular case of infinite inclusion patterns it was shown in Franciosi et al. (2019) that when the pattern evolution is well accounted for, its distribution symmetry can be kept constant and determined by the influence zone symmetry around each fiber element as far as the symmetry and anisotropy characteristics of the embedding matrix do not change.

In particular, applying Eq. (16b) to the effective property estimate for matrix-embedded FPAs in planes of normal $x j$ (whether $j$ is 1 or 3 ) with $x 2$-oriented fibers (corresponding to $\theta=0$ as counted in the FPA plane), to be denoted $\boldsymbol{C}_{\text {effPCW }}^{x j F P(0)}$, the estimate is

\footnotetext{
${ }^{9}$ Spatial distributions of inclusions are unlikely ellipsoidal and are unlikely multimodal at non dilute concentrations (Franciosi and Lebail, 2004). It is not proved that Eq. (16a) does not hold for a non ellipsoidal distribution symmetry.
}

taken of the form:

$$
\boldsymbol{C}_{e f f P C W}^{x j F P A(0)}=\boldsymbol{C}^{M}-f_{f i b}\left(\left(\left(\boldsymbol{C}^{M}-\boldsymbol{C}^{f i b}\right)^{-1}-\overline{\boldsymbol{t}_{\boldsymbol{C} M}^{x j \infty F P(0)}}\right)+f_{f i b} \boldsymbol{t}_{\mathbf{C}^{M}}^{p x j}\right)^{-1},
$$

where the representative FPA mGO $\overline{\boldsymbol{t}_{\mathrm{C}^{M}}^{x j \infty P A(0)}}$ is either the C-fiber one or the SQ-beam one as recalled in Section 2.2 and with $\boldsymbol{t}_{\mathbf{C}^{M}}^{\text {pxj }}$ the laminate (also called platelet) uniform GO referring to the matrix phase $M$. Since in the following only infinite FPAs will be referred to in building pile ups, the symbol infinite will be skipped unless a request of recalling it (and if finite FPAs need be mentioned, they will be noted as nFPAs).

If this FPA-reinforced matrix is deformed in such a way that the interior characteristics of the FPA evolve (for example the global orientation or the fiber inter distance in it) the Eq. (16c) allows to follow the changes of estimated properties due to the FPA evolution accounted for through the mGO. This is a simple example of how the effective properties to be described next for the P-I composite type under study will be followed when some applied deformation mode (as extension) is expected to modify the embedded fiber pattern geometry: this evolution will be carried by the one of the fiber network mGO, driven by the so called "geometrical descriptors" which evolve with the applied deformation as explained in the following section.

\section{Architecture, mGo and effective properties for the pantographic-inspired composite}

Thanks to the availability of mGOs for infinite FPAs of either C-fibers or SQ-beams with any inter-distance value between elements, we can attempt to model the P-I composite type here of concern from embedding in a soft matrix a layered fiber structure whose representative pattern basically consists in such two parallel FPAs with fibers currently oriented along two $2 \theta$-misoriented directions, this misorientation evolving under extension from initially orthogonal $(2 \theta=\pi / 2)$ to a minimal angle when the fibers in each FPA get into contact (null inter distance). Two slightly different routes can be followed for so doing, according to whether the two types of FPA alternated $L-R, R-L$ layers are individually or "simultaneously" embedded in the matrix phase.

In the first choice, a "classical" procedure to perform a homogenization-based modeling of such a two-phase (fiber network plus matrix) composite structure is similar to the one for a laminate structure with alternated layers (Postma, 1955; Christensen, 1979; El Omri et al., 2000). Owing to the heterogeneous structure of the individual layers, this procedure would be a two-level homogenization route, estimating first the effective properties for each layer type and then the effective properties of the laminate structure built from such alternated layers. Effective properties of laminated structures have an exact solution from the used homogenization method when the phase (the layers) properties are exactly known, otherwise (say, if the layers have only estimated properties), it is also an estimate. Yet, it is known that, grossly speaking, effective properties of a laminate elastic structure are close to the Reuss lower bound for the properties normal to the layers while they attain the Voigt upper bound within the layers plane (Voigt, 1889; Reuss, 1929). As shown in the following, 
the difference between the effective properties of two-phase laminates and the Voigt upper bound, which only concerns the properties normal to the layers since the in-layer properties are Voigt-like owing to the parallel arrangement of the phases, results from cross coupling terms between the layers. But these terms do not represent any bundle-like stiffness through-layer (possibly inclinationdependent) contribution to the overall stiffness of the composite corresponding to the 3D networks of concern, since it is easily verified that the layers will not remain connected if the embedding matrix has vanishing stiffness. In the particular present case of layers only differing by an in-plane orientation, the layer properties normally to the layers remain uniform and directly yield the effective properties of the structure in that normal direction (said differently, Voigt and Reuss bounds coincide for the properties normal to the layers).

A relevant modeling alternative (allowed by the fact that the "embedded" phase is co-continuous with the matrix) consists in substituting the laminate scheme on the two preliminary estimated effective properties for each layer type with a simple arithmetic averaging. In contrast with the laminate scheme, this simpler estimate does not generate, owing to its construction, any throughlayer cross coupling terms. But since the network layers have uniform properties with regard to the normal direction to the layer plane, the arithmetic average is in that normal direction just the uniform normal properties of all layers. Consequently, both these two modeling procedures identically give Voigt effective properties within the layers and uniform ones normally to them, say normally to the FPAs.

Attempting to "simultaneously" embed a pair of $\pm \theta$ misoriented FPAs is only possible from the averaging route and amounts to first constructing a global mGO for a pantographic ( $L-R$ or $R-L$ ) bilayer from an arithmetic averaging of mGOs of the two constitutive FPAs which are currently rotated by angles of $\pm \theta$ and to then estimating the effective properties of a two-phase (matrix plus P-I fiber network) composite, the fiber pattern of which is represented by this mGO average.

But none of these descriptions of FPA assemblages accounts for more transversal stiffness than the matrix-due one, what calls for additionally introducing in the structure a specific description of through-layer interconnections. As commented in the beginning, through-layer interconnections of the pantograph pivot type can be mimicked by a sort of third $1 \mathrm{D}$ w-oriented fiber bundle going through the layers, corresponding to the interconnecting pivot arrays which are more or less fiber-like aligned "in the average", even if their arrays can become inclined and even if they are expected to twist or/and bend alternately between layer pairs as commented and illustrated later on. Of course, such through-layer interconnections between the fibers of each neighboring two layers in the ... $L-R-L-R \ldots$ piling will also have determinant in-plane effects on the behavior and on the evolution of the structure under external loading or straining. Accounting for these in-plane effects consists in considering the geometric constraints implied by such interconnections on these structural behavior and evolution. This description of in-plane effects of the through layer interconnections is a fundamental point in the here proposed description: the interconnection mechanics rules the structure deformation (extension or shear) in simultaneously modifying the relative angles $2 \theta$ of the alternated layers and the in-layers fiber inter-distance (say the in-layer FPA compactness as defined by the ratio $\mathrm{R} / \mathrm{L}$ for $\mathrm{C}$-fibers of radius $\mathrm{R}$ or a/L for SQ-beams of side a), which in turns also prescribe the strengthening (torsion-like and bending-like) contributions to be accounted for according to the layer interconnection strength and characteristics.

Note that owing to a generally non zero inter distance $(h)$ between successive layers in a pile, the alternated FPAs could be considered as separated by some fiber-free matrix layers through which only the layer (1D fiber bundle-like) interconnectors would cross. For sake of keeping simplicity in the description no third layer type is added into the laminate description (otherwise specific Eqs. (17c), (19) and related ones for two-phase structures would not hold anymore) and these variable densities of the fiber layers in the composite will be accounted for in varying the matrix volume fraction that embeds each of the two $\pm \theta$ fiber layer families, through the expression of the mean fiber density within the structure, as described in the last part of this section. It is noteworthy that this way to account for the layer inter distances in terms of fiber volume fraction in the structure does not represent any fiber pair interaction from layer to layer which are disregarded here on the argument that interactions between misoriented FPAS are expectedly low compared with cumulated interactions between parallel fibers in FPAs. This may be questionable in situations when the fiber in-layer inter distance is large while the layer piling is dense, that is essentially at the beginning of a deformation process applied to the structure, hence of no much expected consequence if ignored. Comparisons with numerical simulations will show that the neglect of these FPA interactions is indeed reasonable.

Hence, prior to considering additional through-layer interconnections and prior to describing the in-plane features of a pantographic-like deformation mode, the two procedures here finally considered to formulate first effective properties for piled FPAs in a matrix start as follows:

i. Considering first the composite as constituted by two misoriented FPA-reinforced matrix layers, each one having its effective properties calculated first from the $\mathrm{MGO}$ of its C-fiber or SQbeam FPAs (using the appropriately rotated form of Eq. (16c)) and secondly applying the homogenization procedure for calculating the effective properties of the equivalent homogeneous materials to this biphasic laminate material, using Eq. (16b) also in the appropriate form (the alternative of averaging the effective properties first obtained for the two FPA-reinforced matrix at the place of using the laminate scheme is abandoned);

ii. Determining first, from the knowledge of the mGOs for FPAs of C-fibers or SQ-beams reported in Section 2.2 (Eqs. (13-15), the $\mathrm{mGO}$ for the P-I fiber bi-layer alternated assemblage as a simple arithmetic average of the two $\pm \theta$ FPA mGOs, this average mGO being taken as the one of the representative pattern for the P-I fiber networked phase and then using directly this newly built P-I fiber network mGO in the derivation of the P-I composite effective properties, still using Eq. (16b) in appropriate manner.

Presentation of both procedure foundations have shown that they coincide for the description of the P-I composites here of concern which keep uniform properties normally to the FPA layers. The common additional steps of introducing some through-layer inter connections and the in-layer pantograph-like geometrical descriptors are presented next.

\subsection{Effective properties of P-I composites from a two-layer FPA-based laminate scheme}

In the laminate-based description, we still consider a compliant matrix with stiffness tensor $\boldsymbol{C}^{M}$ embedding, according to various "networked" configurations, a single fiber phase with stiffness tensor $\boldsymbol{C}^{F}$ and volume fraction $f_{F}$. A simple laminate structure of these two phases (say made of homogeneous layers of each one) with $\omega_{j}$-oriented normal has effective stiffness estimate that reads from specializing Eq. (16b):

$\boldsymbol{C}_{e f f}^{\operatorname{Lam}\left(\omega_{j}\right)}=\boldsymbol{C}^{M}-f_{F}\left(\left(\boldsymbol{C}^{M}-\boldsymbol{C}^{F}\right)^{-1}-\boldsymbol{t}_{\boldsymbol{C}^{M}}^{p \omega_{j}}+f_{F} \boldsymbol{t}_{\boldsymbol{C}^{M}}^{p \omega_{j}}\right)^{-1}$,

with $\boldsymbol{t}_{\mathbf{C}^{M}}^{p \omega_{j}}$ the uniform laminate (platelet) GO of normal direction $\omega_{j}$ referring to the matrix phase $M$. This is exemplified in Fig. 9 for 


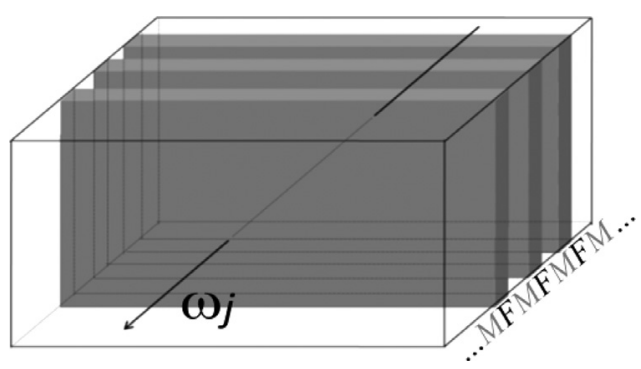

Fig. 9. An elementary two-phase (M,F) laminate structure (alternated ...M,F,M... layers are not necessarily of same thickness, only the phase volume fraction matters in Eqs. (17).

the $x_{3}$ axis as $\omega_{j}$ orientation with $\boldsymbol{t}_{\mathbf{C}^{M}}^{p \times 3}$ given in Table 3 last line. All phases playing equivalent roles in laminate structures, Eq. (17a) is equivalent to referring to phase $F$ as the matrix, $f_{M}=1-f_{F}$, as:

$\boldsymbol{C}_{e f f}^{\operatorname{Lam}\left(\omega_{j}\right)}=\boldsymbol{C}^{F}-f_{M}\left(\left(\boldsymbol{C}^{F}-\boldsymbol{C}^{M}\right)^{-1}-\boldsymbol{t}_{\boldsymbol{C}^{F}}^{p \omega_{j}}+f_{M} \boldsymbol{t}_{\boldsymbol{C}^{F}}^{p \omega_{j}}\right)^{-1}$.

In Eq. (17), the laminate (uniform) GO appears twice, according to the PCW estimate: the negative full occurrence stands for the layers taken as embedded domains and the positive fractional one stands for the "shape" (the symmetry) of the spatial distribution of these layer-like inclusions. Both these shapes are homothetic (and thus have the same operator) since distributing spatially infinite parallel layers can only be done according to such a laminated arrangement. Also, Eq. (17) identify to the two HashinShtrikman bounds (Hashin-Shtrikman, 1963) for the phases $M$ and $F$ which only coincide for this case of a $1 D$ laminate structure of the phases (hence, all other relevant, necessarily in-between, estimates also coincide for $n$-phase laminates, $\forall n \geq 2$. The symmetric form that clearly shows this identity for laminates, discussed first in Franciosi et al. (2011) to the authors knowledge, reads:

$$
\begin{aligned}
\boldsymbol{C}_{e f f}^{\operatorname{Lam}\left(\omega_{j}\right)} & =\boldsymbol{C}+\left(\{\boldsymbol{C}\}-\boldsymbol{C}^{M}\right): \boldsymbol{t}_{\{\boldsymbol{C}\}}^{p \omega_{j}}:\left(\{\boldsymbol{C}\}-\boldsymbol{C}^{F}\right) \\
& =\boldsymbol{C}-f_{M} f_{F}\left(\boldsymbol{C}^{F}-\boldsymbol{C}^{M}\right): \boldsymbol{t}_{\{\boldsymbol{C}\}}^{p \omega_{j}}:\left(\boldsymbol{C}^{F}-\boldsymbol{C}^{M}\right)
\end{aligned},
$$

with $\{\boldsymbol{C}\}=f_{M} \boldsymbol{C}^{F}+f_{F} \boldsymbol{C}^{M}$ and $\langle\boldsymbol{C}\rangle=f_{F} \boldsymbol{C}^{F}+f_{M} \boldsymbol{C}^{M}$ (the Voigt average). The key property of this symmetric form, which is at the origin of the laminate (or platelet) system scheme of homogenization for composites with co-continuous phases, is the reference third phase of properties $\{\boldsymbol{C}\}$ which acts as a reference matrix of infinitesimal volume fraction embedding both phases $F$ and $M$ and in which the uniform laminate GO is defined.

For a bi-layered laminate structure based on associating two \pm $\theta$-rotated FPA in the plane of normal $x_{1}$, each layer family will have effective properties obtained after rotation of Eq. (16c) as:

$\boldsymbol{C}_{\text {effPCW }}^{x_{1} F P A( \pm \theta),}=\mathcal{R}_{i p}( \pm \theta) \mathcal{R}_{j q}( \pm \theta) \mathcal{R}_{k r}( \pm \theta) \mathcal{R}_{l s}( \pm \theta) \boldsymbol{C}_{\text {effPCW }}^{x_{1} F P A(0)}$,

where $\mathcal{R}( \pm \theta)=\begin{array}{rcc}1 & 0 & 0 \\ 0 & \cos ( \pm \theta) & -\sin ( \pm \theta)) \text {. Using these rotated } \\ 0 & \sin ( \pm \theta) & \cos ( \pm \theta)\end{array}$ stiffness tensors for both constitutive $\pm \theta$ layer families of the laminate structure, the effective properties of the P-I composite can be written as those of a laminate made of FPA alternated layers according to Eq. (17c):

$$
\begin{aligned}
& \boldsymbol{C}_{\text {effLam }}^{x 1 \text { PANT }( \pm \theta)}=\left\langle\boldsymbol{C}_{\text {effPCW }}^{x 1 \text { PPA }( \pm \theta)}\right\rangle
\end{aligned}
$$

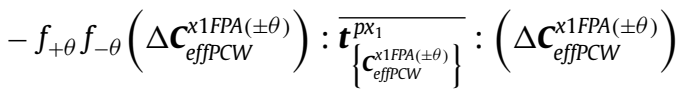

with:

$\left\langle\boldsymbol{C}_{e f f P C W}^{x 1 F P A( \pm \theta)}\right\rangle_{f_{+\theta} / f_{-\theta}}=f_{+\theta} \boldsymbol{C}_{e f f P C W}^{\chi 1 F P A(+\theta)}+f_{-\theta} \boldsymbol{C}_{e f f P C W}^{\chi 1 F P A(-\theta)}$
$\Delta \boldsymbol{C}_{e f f P C W}^{\chi 1 F P A( \pm \theta)}= \pm\left(\boldsymbol{C}_{e f f P C W}^{\chi 1 F P A(+\theta)}-\boldsymbol{C}_{e f f P C W}^{\chi 1 F P A(-\theta)}\right)$

and:

$\left\{\boldsymbol{C}_{e f f P C W}^{\chi 1 F P A( \pm \theta)}\right\}_{f_{+\theta} / f_{-\theta}}=f_{-\theta} \boldsymbol{C}_{e f f P C W}^{\chi 1 F P A(+\theta)}+f_{+\theta} \boldsymbol{C}_{e f f P C W}^{\chi 1 F P A(-\theta)}$.

Since $\left\langle\boldsymbol{C}_{e f f P C W}^{x 1 F P A( \pm \theta)}\right\rangle_{f_{+\theta} / f_{-\theta}}$ is nothing else than the Voigt estimate (arithmetic average) for this bi-layered structure as defined, the deviation of the laminate estimate $\boldsymbol{C}_{\text {effLam }}^{x 1 P A N T}$ from the Voigt upper bound is fully carried by the second part in Eq. (19). This second part is only non-zero for terms related to

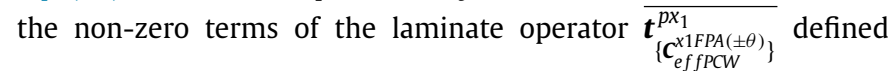
on the reference medium with properties $\left\{\boldsymbol{C}_{\text {effPCW }}^{x 1 F P A( \pm \theta)}\right\}_{f_{+\theta} / f_{-\theta}}$. Note that equal fractions of both layer types yield $\left\{\boldsymbol{C}_{e f f P C W}^{x 1 F P A( \pm \theta)}\right\}_{f_{ \pm \theta}=0.5}=$ $\left\langle\boldsymbol{C}_{e f f P C W}^{\times 1 F P A( \pm)}\right\rangle_{f_{ \pm \theta}=0.5}$, that is the stiffness tensor of the reference matrix phase equals the Voigt stiffness estimate for the twophase structure, and that the layers are not orthotropic in a same frame unless $2 \theta=90^{\circ}$, what yields non zero skew-symmetric parts. Writing $\boldsymbol{C}^{x 1 F P A( \pm \theta)}=\boldsymbol{C}^{O(\theta)} \pm \boldsymbol{C}^{S S(\theta)}$ to separate the symmetric (orthotropic "O") and the skew-symmetric "SS" parts in these oppositely rotated tensors, the latter ones cancel each other by $\pm \theta$ symmetry such that while $\left\{\boldsymbol{C}_{e f f P C W}^{x 1 F P A( \pm \theta)}\right\}_{0.5 / 0.5}=\left\langle\boldsymbol{C}_{e f f P C W}^{x 1 F P A( \pm \theta)}\right\rangle_{0.5 / 0.5}=$ $\boldsymbol{C}^{O(\theta)}$ keeps orthotropic symmetry, one has $\Delta \boldsymbol{C}_{e f f P C W}^{x 1 F P A( \pm \theta)}= \pm 2 \boldsymbol{C}^{S S(\theta)}$ in Eq. (20b) where the sign of $\triangle \boldsymbol{C}_{e f f P C W}^{x 1 F P A( \pm \theta)}$ does not matter for only square terms of it appear in the formula of Eqs. (19) or (17c). It is not in the present scope to further examine the specificities of such structures, yet it is easily verified, with helping details in Appendix C, that owing to the property uniformity of the $R$ and $L$ layers with regard to their normal direction the laminate normal properties remain the common normal properties of both layer types.

\subsection{Effective properties of $P$-I composites from the $m G O$ for a two-layer FPA pattern}

In the stiffness averaging procedure, the effective property estimate of the P-I composite from the construction of a global mGO for the P-I 3D fiber network in it consists in:

i. Rotating the FPA mGO with generic form given Section 2.2 in Table 6 at the two opposite angles of $\pm \theta$ (from $\pm \pi / 4$ to non zero minimal $\pm \theta^{*}$ values depending on the minimal distance between fiber interconnections) using Eq. (18) on the mGOs;

ii. Calculating the $\mathrm{mGO}$ for the P-I network (to be denoted $\overline{\boldsymbol{t}_{\mathbf{C}^{M}}^{x \text { Pant }(\theta)}}$ where $\mathrm{xj}$ indicates the normal direction to the layers and $\theta$ the misorientation characteristic angle) from arithmetic averaging those of the two $\pm \theta$ misoriented FPAs;

iii. Using this mGO for estimating effective properties in appropriately using Eq. (16b) where this network mGO will stand for the operator $\overline{\boldsymbol{t}_{\boldsymbol{c}^{M}}^{V}}$, the relevant distribution $\boldsymbol{t}_{\mathbf{c}^{\mathrm{M}}}^{\text {SDist }} \mathrm{GO}$ still being the uniform laminate $\boldsymbol{t}_{\mathbf{c}^{M}}^{p x j}$ GO from appropriately rotating last line of Table 3.

The mGOs of the $\pm \theta$ rotated FPAs around the direction $x_{1}$ are of the form:

$\overline{\boldsymbol{t}_{\mathbf{C}^{M}}^{x 1 F P A( \pm \theta)}}=\mathcal{R}_{i p}( \pm \theta) \mathcal{R}_{j q}( \pm \theta) \mathcal{R}_{k r}( \pm \theta) \mathcal{R}_{l s}( \pm \theta) \overline{\boldsymbol{t}_{\mathbf{c}^{M}}^{x 1 F A(0)}}$,

where $\mathcal{R}( \pm \theta)$ has been given and $\overline{\boldsymbol{t}_{\mathbf{C}^{M}}^{x 1 F A(0)}}$ is the sum of the interaction part from Table 6 and of the interior part from Table 3, specializing the generic form for C-fibers or SQ-beams in using the appropriate $C 2 l(x)$ functions, $l=1,2$, from Section 2.2. 


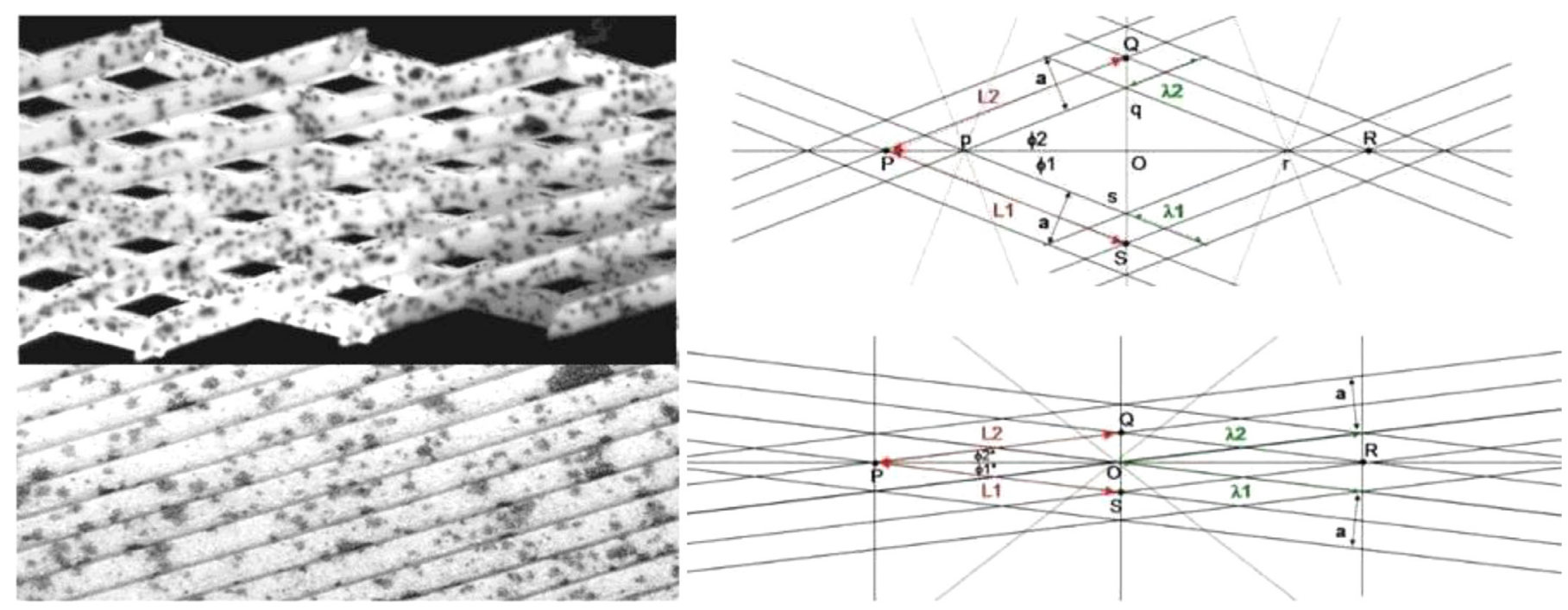

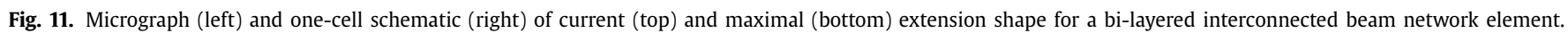

equaling the extension of the fiber network with the composite one and the fiber network evolution under extension totally fixes the current effective properties of the P-I composite through the evolution of its mGO.

\subsection{Geometric descriptors of the constraints on the in-plane pantographic-like deformation}

One now describes the so called in-plane "geometrical descriptors" for the behavior under extension of that two-phase bicontinuous P-I composite structure type which are due to such existing inter connections. As for the chosen description of the through-layer interconnections, the description we use for these in-plane geometrical descriptors is compatible with the two examined and compared (laminate-based and mGO averaging) procedures for estimating the properties of the composite of concern. The Fig. 11 right which shows a top view of a RVE for the fiber network into two extension positions, explicate how the geometric descriptors are linked and drive the evolution of the network, such as to rule the evolution of the effective elastic properties for the P-I composite through the Eq. (23). We consider, as drawn according to the micrographs of Fig. 11 left a pair of $\left(\varphi_{1}, \varphi_{2}\right)$ oppositely oriented beam layers with regard to the horizontal axis, with all beams of same rectangular cross section area $a \cdot b$, where $b$ is the height and $a$ the in-layer width, such that $b=a$ for SQ-beams (this discussion is valid also in case of cylindrical fibers with cross section area $\pi R^{2}$ and radius $R$ ). Along each beam, regularly spaced attachments ("pivots") inter-connect at inter distance $L$ the successive two (or more, above and below) alternated layers. The beams are considered as undeformable such that $a, b$ and $L$ are kept constant. The details of the pivot geometry are at first disregarded and their height $(h)$ - which is the inter-distance between the layers - is just supposed to allow beam rotations without affecting the structure, although behavior differences are highly expectable whether (h) is small (dense layer piling) or large (distant layers).

Thus, as viewed along the normal to the layers ("top view"), the four interconnecting pivots of an elementary representative domain are firstly reduced to points (Fig. 11a,b), as the $P Q R S$ set from Fig. 4, such that we a priori disregard possible deformation of the pivots as bending (distortion from straight cylinders) or inclination with regard to the $\boldsymbol{z}$ normal to $\boldsymbol{x}$ - $\boldsymbol{y}$ layers (say the $\boldsymbol{w}$ orientation is kept parallel to $\boldsymbol{z}$ ). In ideal symmetric and homogeneous extension along a line going through $P$ and $R$ (a priori neglecting also beam distortions at the pivot level as well as between pivots along beams), the main current characteristic of the elementary representative cell $P Q R S$ is that the two diagonal segments $P R$ and $Q S$ remain orthogonal (Fig. 11a), with $L_{1}=L_{2}=L$ and $\varphi_{1}=-\varphi_{2}=\theta$ (for notation consistency with previous sections). If the two beam layer types are initially orthogonal, the decrease of $\pm \theta$ ranges from $\pm \frac{\pi}{4}$ to minimal $\pm \theta^{*}$ angles. In terms of beam misorientations, the current length of the network is given by $P R=2 L \cos \theta$, from $\sqrt{2} L$ and the current structure extension is:

$\alpha=\frac{P R-P R_{0}}{P R_{0}}=\sqrt{2} \cos \theta-1$.

The current area of the PQRS "cell" is $\mathcal{A}_{P Q R S}=\frac{1}{2} P R \cdot Q S=$ $2 L^{2} \cos \theta \sin \theta=L^{2} \sin 2 \theta$. Similarly, the area of the commonly "free" interspace pqrs between the fibers of successive layers is given by $\mathcal{A}_{\text {pqrs }}=\frac{1}{2} \mathrm{pr} \cdot q s=(L-\lambda)^{2} \sin 2 \theta$, where $\lambda=a / \sin 2 \theta$ is the side length of the beam superposition area and is equal to $a$ initially.

Consequently, the inter-distance $(d)$ between any two parallel beams varies with $\theta$ as:

$d=(L-\lambda) \sin 2 \theta=L \sin 2 \theta-a$,

and when beams are at contact (Fig. 11b), $\mathcal{A}_{\text {pqrs }}=0$ and $\lambda=L$ such that $d=0$ and the minimal misorientation is $\theta^{*}=\frac{1}{2} \sin ^{-1} \frac{a}{L}$. The maximal extension follows as:

$\alpha^{*}=\sqrt{2} \cos \theta^{*}-1=\sqrt{2} \cos \left(\frac{1}{2} \sin ^{-1} \frac{a}{L}\right)-1$

The limit for the extension when $a / L$ goes to 0 is $\sqrt{2}-1 \approx$ $41,42 \%$ and it is still $41 \%$ when $a / L \approx 0,15$ and $40 \%$ when $a / L \approx 0$, 28. As for the in-plane inter distance $(d)$ between fibers, the inter distance between layers is zero for layers at contact, in which case the inter connecting pivots have a zero height $(h)$. For a general (h) value, an elementary volume element is $\mathcal{V}_{P Q R S}=(h+b) \mathcal{A}_{P Q R S}=$ $(h+b) L^{2} \sin 2 \theta$. For compact layers $(h=0)$, the void part equals twice the interlayer spacing along a segment $L$ :

$\mathcal{V}_{\text {void }(h=0)}=\mathcal{V}_{\text {voidmin }}=2(L d)\left(\frac{b}{2}\right)=b L(L \sin 2 \theta-a)$,

and for $(h)$ being general:

$\mathcal{V}_{\text {void }(h)}=\mathcal{V}_{\text {voidmin }}+h L^{2} \sin 2 \theta=(b+h) L^{2} \sin 2 \theta-b L a$. 
Thus, the void volume fraction, which also is the embedding matrix volume fraction, reads:

$f_{\text {void }(h)}=\frac{(b+h) L^{2} \sin 2 \theta-b L a}{(h+b) L^{2} \sin 2 \theta}=1-\frac{b a}{(h+b) L \sin 2 \theta}$,

with $f_{\text {void }(h=0)}=1-\frac{a}{L \sin 2 \theta}=\frac{L-\lambda}{L}=1-\frac{a(L-\lambda)}{L d}$.

The fiber volume fraction which appears in Eqs. (16c, 23) conversely has the form:

$f_{f i b(h)}=\left(\frac{b}{h+b}\right) \frac{a}{L \sin 2 \theta}=\left(\frac{b}{h+b}\right) f_{f i b(0)}$.

In the cases when the pivots between fiber layer are allowed to bend (say to become inclined), there is no more equivalency between the pivot height and the layer inter-distance unless initially (no extension yet): if the bending angle $\kappa$ is the one given by the pivot orientation $(\boldsymbol{w})$ and the normal $(\boldsymbol{z})$ to the fiber layers, say $\kappa=(\boldsymbol{w}, \boldsymbol{z})$, the layer inter distance will decrease, possibly down to a null value if that bending can drive the fiber layers to contact. The relation of this bending angle $\kappa$ to the misorientation angle $\theta$ is another geometric descriptor. This bending influence can be also simply examined from considering different values of the layer inter distance $(h)$, as is done next. In the case of SQ-beams (as for rectangular beams with same height $b$ ), the planar alignments at maximal extension become compact laminate layers what corresponds to Fig. 10, with two alternated layer types of all same thickness, and with minimal misorientation $2 \theta^{*}$. The above described geometric changes for a P-I beam or fiber structure explicates the in-plane links between the structure extension, the layer misorientation, the in-plane fiber inter-distances $(d)$ and the fiber layer inter distance $(h)$.

It is noteworthy that any description taking into account the inter distance $(h)$ between FPAs potentially allows to also account for torsion of the pivots, the torsion being in first approximation simply given by the ratio $2 \theta / h$, for the layer inter distance $(h)$ also is the pivot height as long as no bending has occurred. This torsion amount is also connected to the inter distance $(d)$ between the fibers of a layer through the $\theta$ angle current value. This torsion can be further related to a torsional stress contribution provided the knowledge of a torsion stiffness modulus for the pivots which will depend on the pivot structure in real materials. But it can also enter the characteristics of the 1D bundle introduced in the P-I fiber network description, through a variation of stiffness moduli (and especially of the shear modulus) related to the pivot torsion. One can for example consider a stiffness increase (in pure elastic regime without neither plasticity nor damage) with torsion, as $\mu_{\mathrm{B}}(\theta) / \mu_{\mathrm{B}}=\chi(\theta-\pi / 4) \geq 1$, with a function $\chi$ that may be determined from experimental and/or numerical data. In comparison, the pivot bending effect which is the decrease of the FPA inter distance $(h)$ can be more easily considered in the modeling, via the variation law for the fiber volume fraction as explained next on. The various relations synthesized in Eqs. (25)-(30) that we called "geometric descriptors", are pantographic-inspired in the sense that, as described, the interconnections between the FPAs correspond to typical behavior of pantographic structures as for example examined in Andreaus et al., 2018; De Angelo et al., 2019b) among other ones. These relations correspond to the assumption of "fixed pivot" type, already pointed to correspond to a one-placement-field description as discussed in Spagnuolo et al. (2017). Other types of pivots (or fiber layer interconnections) could be considered, in which case other relations would hold between the same geometric descriptors, in place or in addition to the here presented ones. When the elements of the 1D fiber bundle that goes through the FPAs are allowed to become $\boldsymbol{w}$-inclined in the extension direction with regard to the normal of the FPA plane, in the pointed necessary alternated manner at the scale of successive FPA pairs (to be illustrated in Section 4), this corresponds as said already to the two-placement fields assumption examined in Spagnuolo et al. (2017). In the present modeling attempt for the deformation of a P-I composite as defined, an applied extension modifies the geometric descriptors according to the Eqs. (25)((30), from which the effective properties given by Eq. (23b) (or 23a without bundle) can be reactualized to follow the evolutions with extension.

Section 4 first presents the simple behavior description used to explicate the P-I composite effective property variations with an applied extension, through the variation of the fiber network. It then exemplifies analytical results from the proposed extension modeling and the validating with numerical comparisons of the variation of the overall Young and shear moduli follows. At last, comparisons with numerically obtained typical force-displacement curves of 2D pantographs, where the so called pivot strength effects have a variable - from high to low - contribution, establish that the proposed analytical model can integrate these observed effects via several of the explicitly introduced geometric descriptors and, when not enough (namely in case of high pivot strength effects), via a possibly "pivot-strength-induced" variation of the matrix stiffness itself with the FPA misorientation increase. A typical fitting variation law for the matrix shear modulus with the misorientation angle is extracted, as example, from the performed comparisons.

\section{Effective property evolution and force-displacement relation of stretched P-I composite}

The exemplified analytical data result from the use of the averaging procedure method to calculate the $\mathrm{mGO}$ for the pantographic-like bi-layers taken as the representative elements of the matrix-embedded P-I fiber network with using the mGOs of FPAs made of SQ-beam that we previously characterized in the details in (Franciosi, 2018) under the simplifying approximation introduced in Eq. (15) as explained. The modified matrix property form in Eq. (24) is used to account for the trans-layer effective or apparent stiffness of the P-I composite structure, which results from the fiber bundle-like pivot arrays that physically ensures the FPA interconnections and preserves a non vanishing transverse stiffness of the P-I composite.

A first consequence of the alternated $R, L, R, \ldots$ FPA layering in the considered P-I fiber network is the presence of alternated torsions on successive pivots along each array. Although not easily evidenced, neither on numerical nor on experimental experiments (pivots need be marked along their axis to follow that torsion which can be stiff or not depending on the pivot type), this alternated behavior is obvious as can be inferred from looking at Fig. 1 and imagining a stretch of it in the considered extension symmetry conditions. On the contrary, there is a less obvious behavior of the pivots for the same reason of alternated layering, which finds immediate experimental evidence (to be exemplified in the following): the pivot arrays do not strictly remain straightly normal to the FPAs but are alternately bent (become inclined) between successive $R-L$ and $L$-Rlayer pairs such as to remain straightly normal to the layers "in the average".

These pretty much homogeneous trans layer effects (the pivot distribution remains discretely homogeneous during homogeneous extension of the matrix embedded P-I fiber network) are always present in pantographs, in complement to and independently from the more heterogeneous in-layer features which are localized at the interconnections between homogeneous zones and that we disregarded here. Both these two (torsion-due and inclination-due) through-layer features that also result from the in-plane deformation are likely to impact the effective stiffness of the bundle-like pivot array and in turn the overall P-I composite behavior. What means that considering some relevant evolution form for both the 

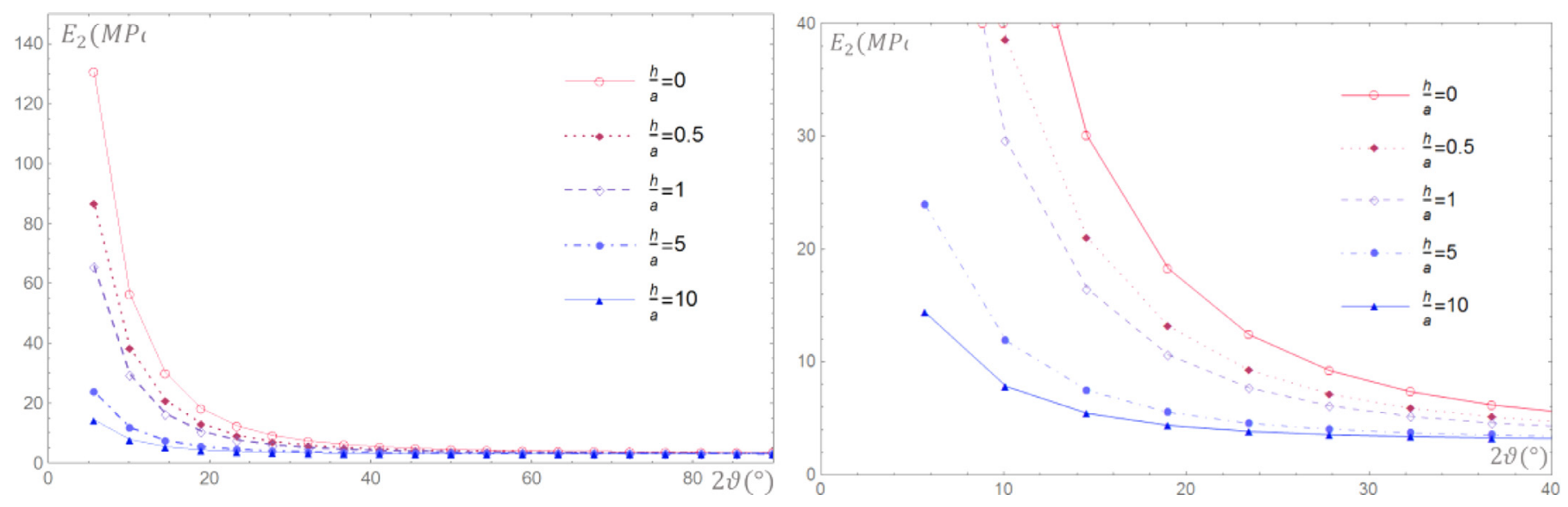

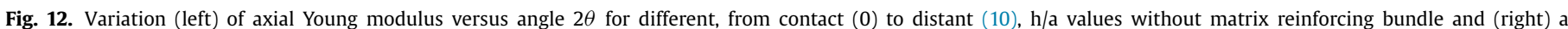
zoom of the left side.
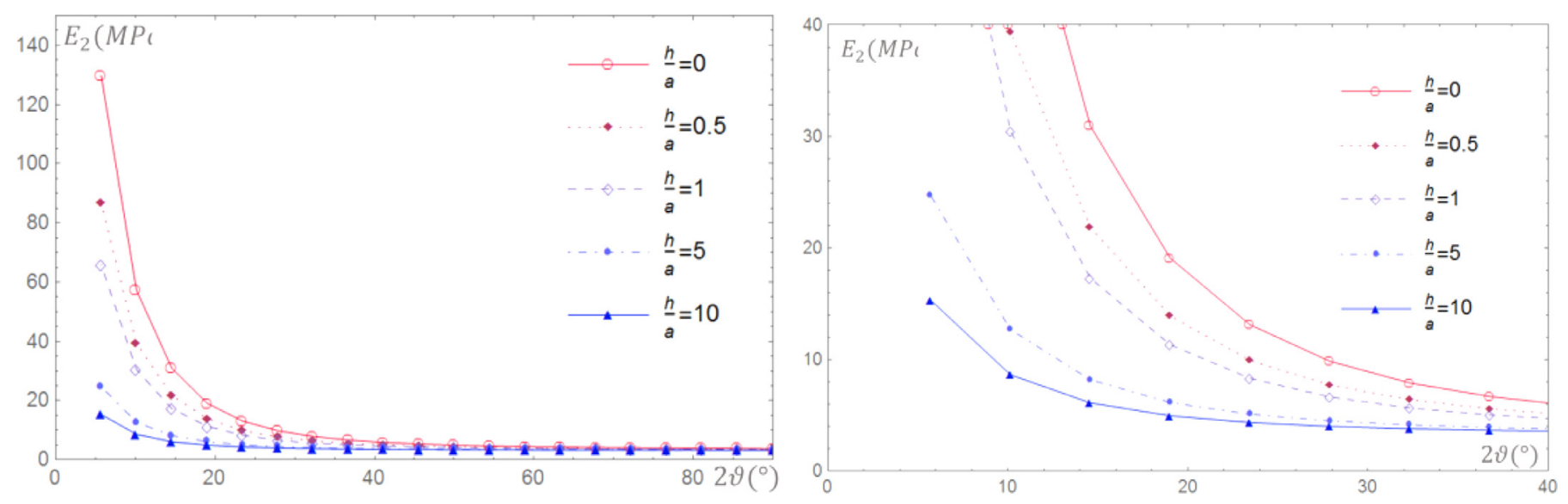

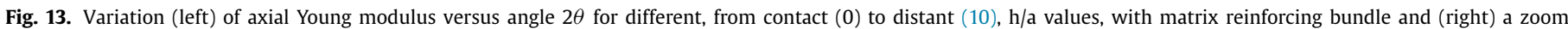
of the left side.

effective stiffness and the geometry of this bundle-like pivot array is a possible way to indirectly account for these important characteristics of the P-I composite structure type, that we globally called "the pivot strength effects" here. Both identified effects involve the layer inter distance $(h)$ which enters the proposed P-I composite descriptions from the expression given to the mean volume fraction of the fiber phase in Section 3.4.

\subsection{Modulus evolutions and force-displacement plot for the pantographic-inspired composite}

In order to simply evaluate the proposed model for estimating effective properties of a P-I two-phase composite as defined, we consider two isotropic and nearly incompressible phases having relative shear moduli of 1 (matrix) and 50 (fibers) with respective Poisson ratio of 0.49 and 0.499 . The fiber-like bundle that represent the pivot array is considered to a priori have the same moduli as the fibers, with considering a possible torsion-due increase of the pivot shear modulus. The initial fiber inter distance $(d)$ is taken equal to 20 times the fiber width $a$.

From using the relations between the so-called geometric descriptors from Section 3.4, giving successive extension states $e_{i}=\Delta L_{i} / L 0$ of the representative cell (PQRS) of the structure, allows two simple calculations:

- It allows on the one hand the determining of the misorientation angles $\pm \theta\left(e_{i}\right)= \pm \theta_{i}$ and from them the fiber inter distance $d\left(e_{i}\right)=d_{i}$ in the layers, that is the two necessary terms allowing to determine effective elastic properties $\boldsymbol{C}_{\text {effPCW }}^{x 1 \text { Pant }\left(\theta_{i}, h\right)}$ according to the averaging description of Eq. (23) and comparing them for a few different values for the $h / a$ layer relative inter distance. This calculation provides the effective axial Young modulus for the extended structure $Y\left(e_{i}, h\right)$ as well as all other effective moduli at successive elastic extension steps, without more incremental precision of the calculations owing to the many simplifying approximates assumed all along the performed analysis for that complex network structure;

- It allows on the other hand the similarly simple calculation of the axial (logarithmic) strain in the PQRS cell $\varepsilon\left(e_{i}\right)=\ln \left(1+e_{i}\right)$, from which an axial stress $\sigma\left(e_{i}, h\right)$ is evaluated as $Y\left(e_{i}, h\right) \varepsilon\left(e_{i}\right)$ which stands for a tensile force per unit cross section area of the structure, say:

$$
F_{S 0=1}\left(e_{i}, h\right)=\sigma\left(e_{i}, h\right)=\mathrm{Y}\left(e_{i}, h\right) \cdot \ln \left(1+e_{i}\right) .
$$

This force identifies in simplified manner the pantograph behavior to the homogeneous extension of its central zone as simplified in Fig. 4 left (or right, up to a body rotation), what is realizable provided appropriate loading boundary conditions. The Fig. 12 shows the so stepwise estimated axial Young modulus variation with angle $2 \theta$ at different values of $h / a$ from 0 (layers at contact) to 10 using Eq. (23a). The Fig. 13 similarly reports the same axial Young modulus evolution with the additional effect of through-layer fiber bundle reinforcement, according to Eq. (24), that is using the effective property tensor of Eq. (23b). The current volume fraction of fibers in the bundle (or pivots in the bilayers) 

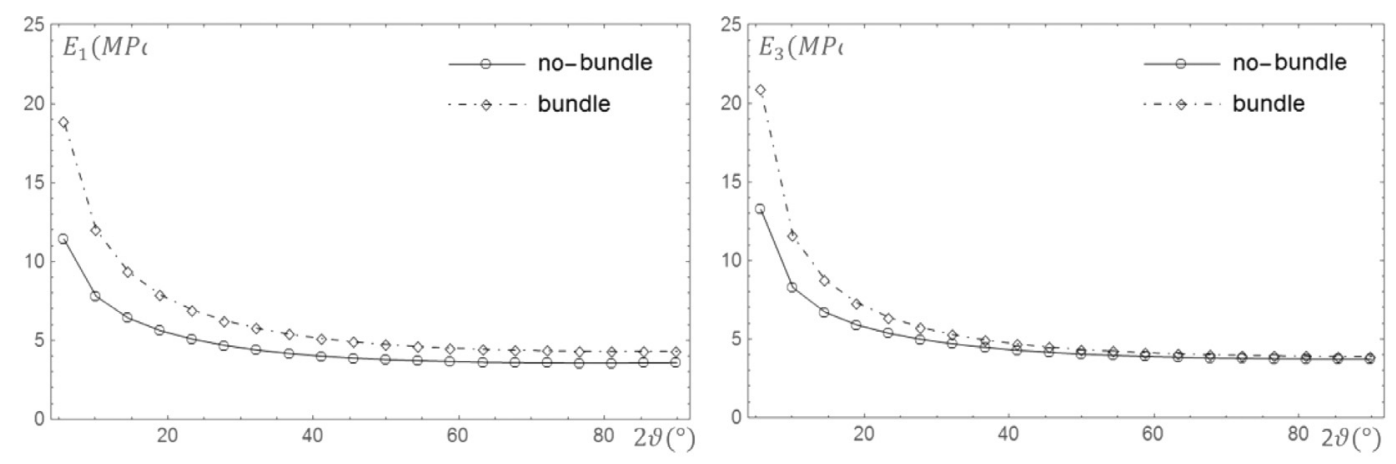

\section{a: Compared evolutions without and with bundle effect at $h=0$ (layer contact) of the normal (left) and the transverse (right) Young modulus with layer misorientation angle $2 \theta$.}
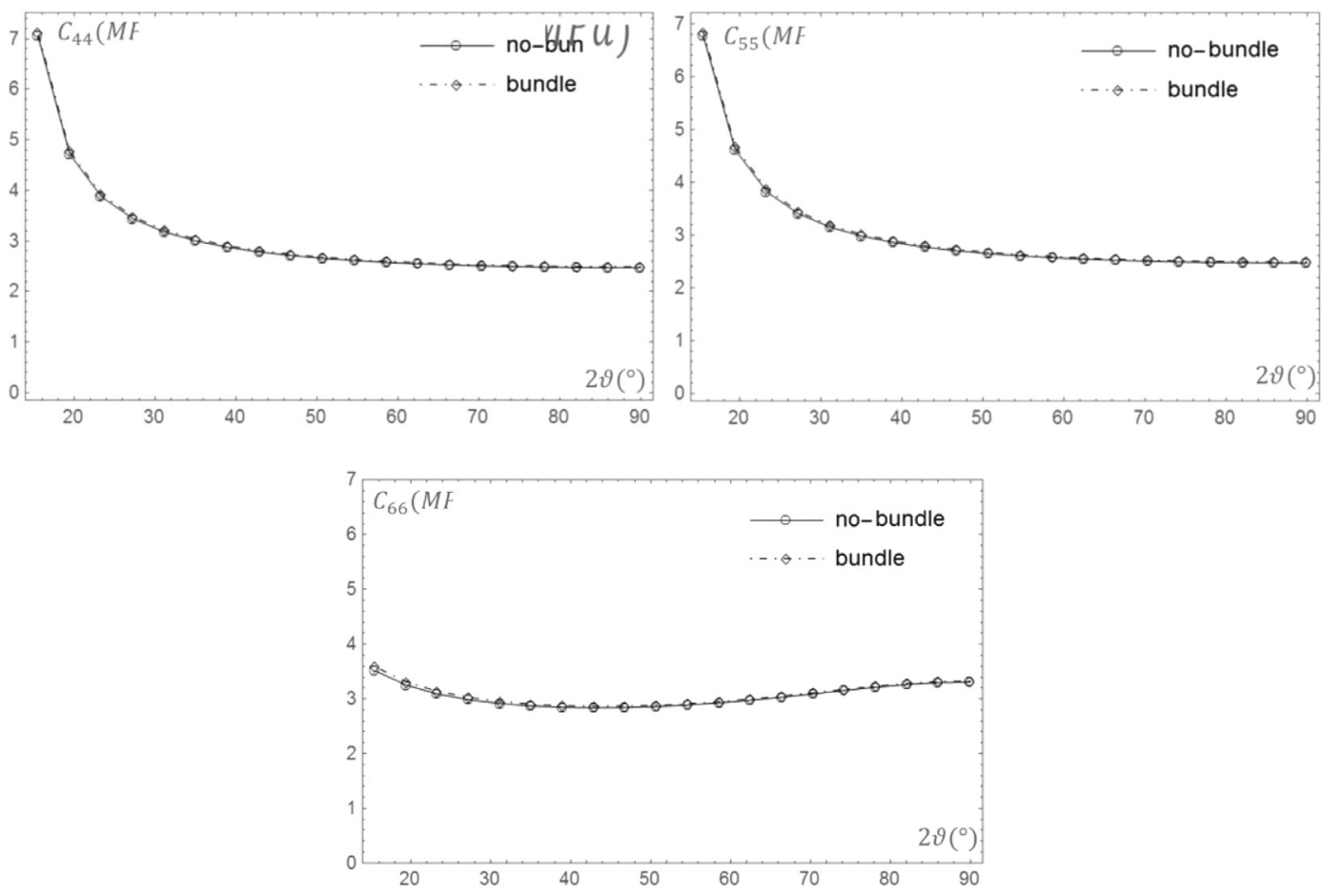

b: Compared evolutions without and with bundle effect at $h=0$ (layer contact) of the lateral $x 1-x 3$ (top, left), the $x 1-x 2$ facial (top, right) and the in-plane $x 2-x 3$ (bottom) shear modulus with layer misorientation angle $2 \theta$.

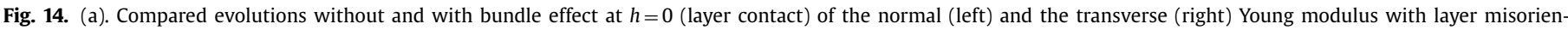

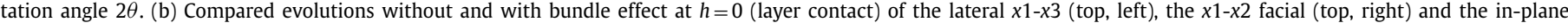
$x 2-x 3$ (bottom) shear modulus with layer misorientation angle $2 \theta$.

is taken as $a^{2} /\left(L^{2} \sin \theta \cos \theta\right)$. The pivot elastic properties are those of the fibers and no additional (torsion-like or inclination-like) effect is considered so far. Since in this example the $a / d$ initial ratio is taken equal to $1 / 20$, the initial low fiber fraction of $25.10^{-4}$ increases to $a / d=5.10^{-2}$ at compact contact and there is no noticeable difference with or without that bundle on the axial Young modulus, say in the extension direction, as expected.

The Fig. 14a,b show the corresponding maximal (i.e. at contact $h=0$ inter distance) variations with $2 \theta$, with and without bundle effect, of the other effective Young and shear elastic moduli, which are much less evolving, in extension, than the axial Young modulus, but with a more pronounced effect especially on the two other (normal and transverse) Young moduli, while none of the 3 shear moduli (along and around the bundle fiber direction) appears affected, as expected too.
The related axial force (per cross section area unit) evolutions, obtained as explained in the beginning of that section from using that axial Young modulus analytical estimate, have the form plotted on Fig. 15 (respectively versus the $2 \theta$ layer angle, left, and versus the normalized extension $\Delta L / \Delta L_{\max }$, right), without and with bundle effect at $h=0$, without appearing noticeable differences either (the extension normalization for the analytical curves uses the maximal extension $/ \Delta L_{\max }$ that corresponds to fiber contact in the FPAs). The force-displacement curves of Fig. 15 right have the well known qualitative form of the force-displacement curves of 2D pantograph prior entering into any plastic or damaged stage. The final sharp increase corresponds to the start of the elastic behavior of the nearly aligned and fully compact FPA families at minimal misorientation angle $\theta$. The next subsection reports some comparisons with numerical validating results. 

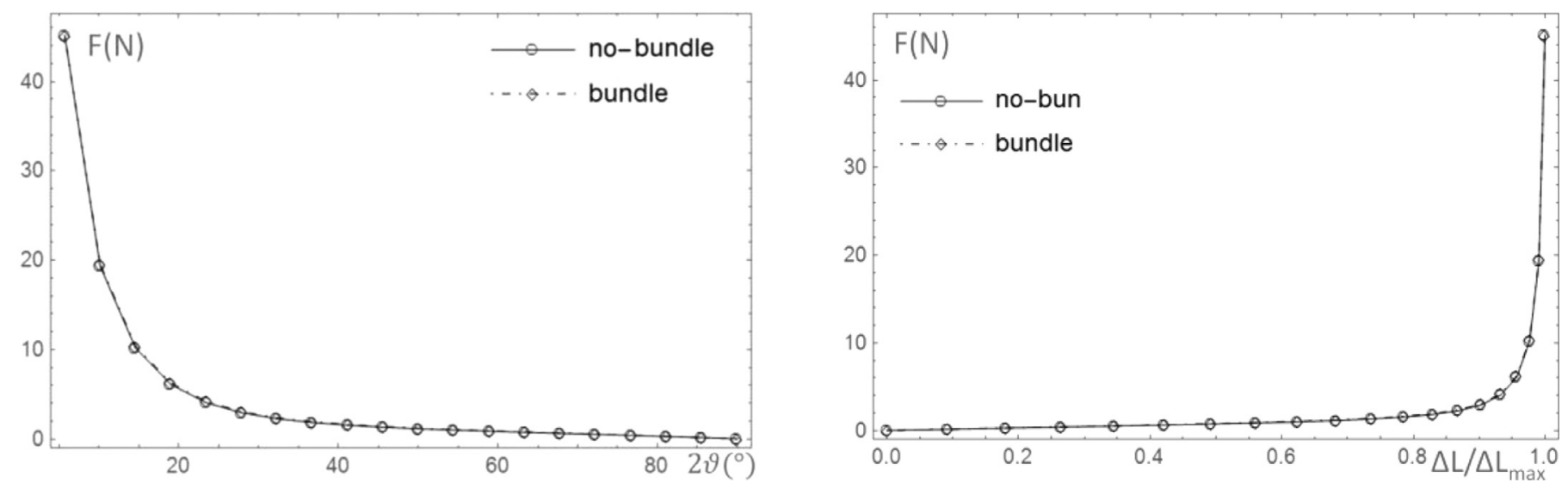

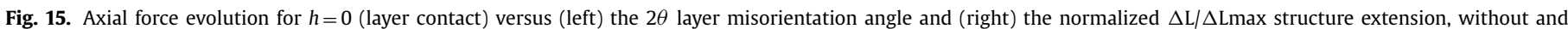
with bundle stiffness contribution.

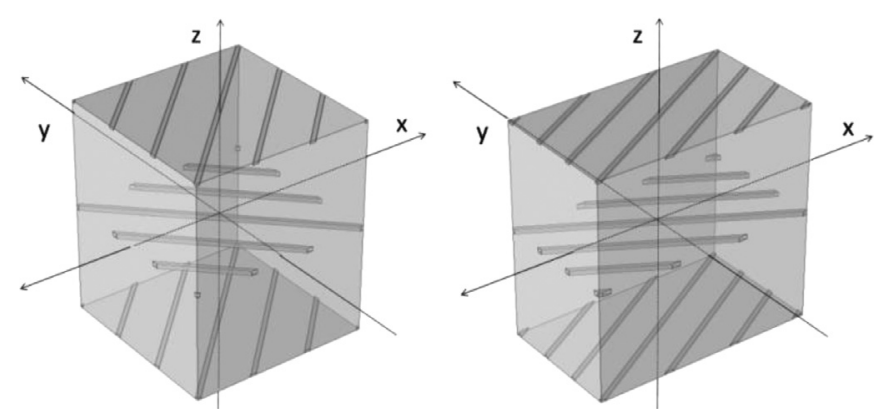

Fig. 16. Initial (left) and $x$-extended (right) periodic cell for numerical estimates of effective modulus evolutions.

\subsection{Compared numerical and analytical elastic modulus evolutions during extension}

Comparisons with numerical simulations are performed from using the cell shown in Fig. 16, in which the layer (relative) interdistance $(h / a)$ is explicitly present. The elastic moduli given to the two phases of that composite cell are the same as for the analytical calculations. There are physically no pivot inter connectors in the cell.

The fiber initial inter distance $(d)$ in the layers is taken to be 20 times the beam side length and the relative layer inter distance $h / a$ is varied from contact ( 0 ) to distant as in the analytical estimates. In order to remain close to the procedure used for the analytical estimate changes with a change of the layer misorientation angle (that is with various extension steps and no distorsion of the structure), the cell structure has been step-wise extended in changing that misorientation angle and on each successive cell with extended structure, 6 elementary strains are applied to evaluate the 3 Young moduli and the 3 shear ones. So doing, the cell remains homogeneously deformed at each extension step, only the fiber network structure being modified and no internal stresses are stored. The first performed calculations are for the variation of the effective axial Young modulus with respect to the FPA relative inter distance $h / a$, from close to distant. These variations are reported in Fig. 17a as a function of the FPA misorientation angle $2 \theta$. The corresponding analytical estimates are also plotted for comparison. It is seen that both calculations pretty well correspond for the $h / a$ values of $(0,1,3)$ which cover the range of typical ratios in pantographs. The Fig. 17b, c similarly report the numerically calculated other (Young and shear) moduli using the same cells, for the case $h=0$ only. These plots show how the tendencies well corresponds with the analytical estimates which are also reported.
The final step of this exploratory work consists in examining possible additional description, in the proposed analytical modeling, of torsion-like and inclination-like strengthening effects on the total force-displacement curve from the pivot arrays, as is the case during in-plane extension of 2D pantographs, that we call for short "pivot strength effects".

\subsection{Compared numerical and analytical force-extension curves with pivot strength effects}

We compare a typical numerical force-displacement curve of a 2D pantograph, which is homogeneously in-plane stretched thanks to appropriately prescribed boundary conditions that suppress the heterogeneous second gradient effects, with the forcedisplacement curves obtained from the presented analytical modeling which is pivot-strength-free as built. The boundary descriptions for these comparative numerical simulations are defined (see Appendix D) such that the whole 2D pantograph is homogeneously strained as the central zones in Fig. 4. So doing, compared with the pure extension that is described in the analytical homogenizationbased model, the numerical one is only complemented with a discretely homogeneous pivot strength field coming from the specific torsion or/and inclination features, using an elementary description. Depending on this pivot strength, the difference between the total force-displacement curve of the pantograph and its extension part can be either dominant or minor. This section stresses the two following results:

i) It is possible to pretty well match an analytically obtained $F / \Delta L$ curve from the proposed modeling, as the one shown in Fig. 15, characterized by a low matrix to fibers stiffness ratio and which is pivot-strength-free as built, in adjusting the pivot strength part in the total pantograph $F / \Delta L$ extension curve;

ii) It is conversely possible to pretty well match a numerical pantograph $F / \Delta L$ curve with low or moderate pivot strength contribution in accounting in the analytical proposed modeling for either a torsion-due stiffness increase of the bundle-like pivot arrays or for an inclination-due decrease of the fiber layer inter distance $(h)$, or for both effects; when these two effects (each remaining of limited influence) prove insufficient (say in case of high pivot strength contribution), a good match is still accessible with assuming the matrix stiffness to increase according to some dependency on the fiber misorientation $2 \theta$, the form of which can be deduced from the analytical versus numerical comparisons. These 3 influences are exemplified using elementary descriptions. 

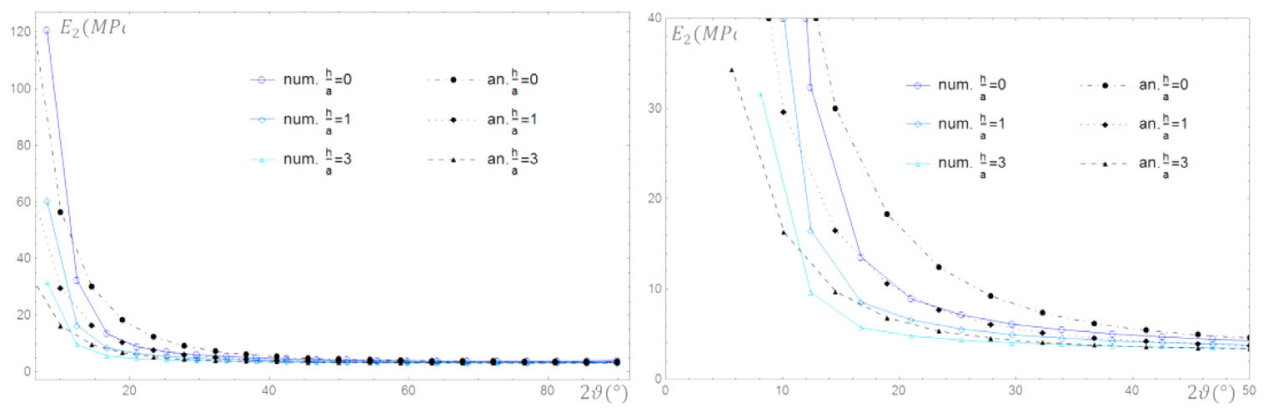

$\boldsymbol{a}$ : Compared (left) numerical and analytical axial Young modulus estimated evolutions with FPA misorientation angle $2 \theta$ at different (h/a) values and (right) a zoom of the left side.

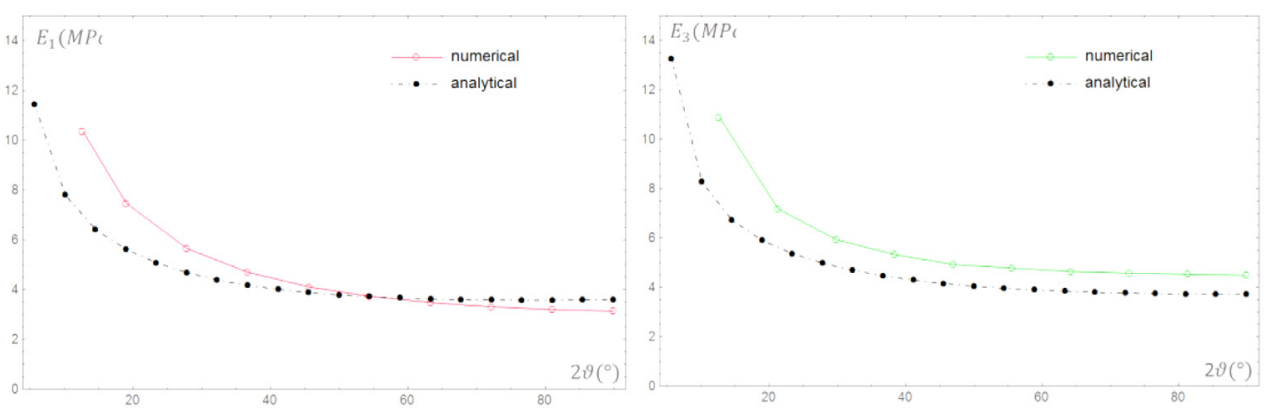

b: Compared numerical and analytical simulations of estimated evolutions with layer misorientation angle $2 \theta$ of the normal (left) and the transverse (right) Young modulus at $h=0$.
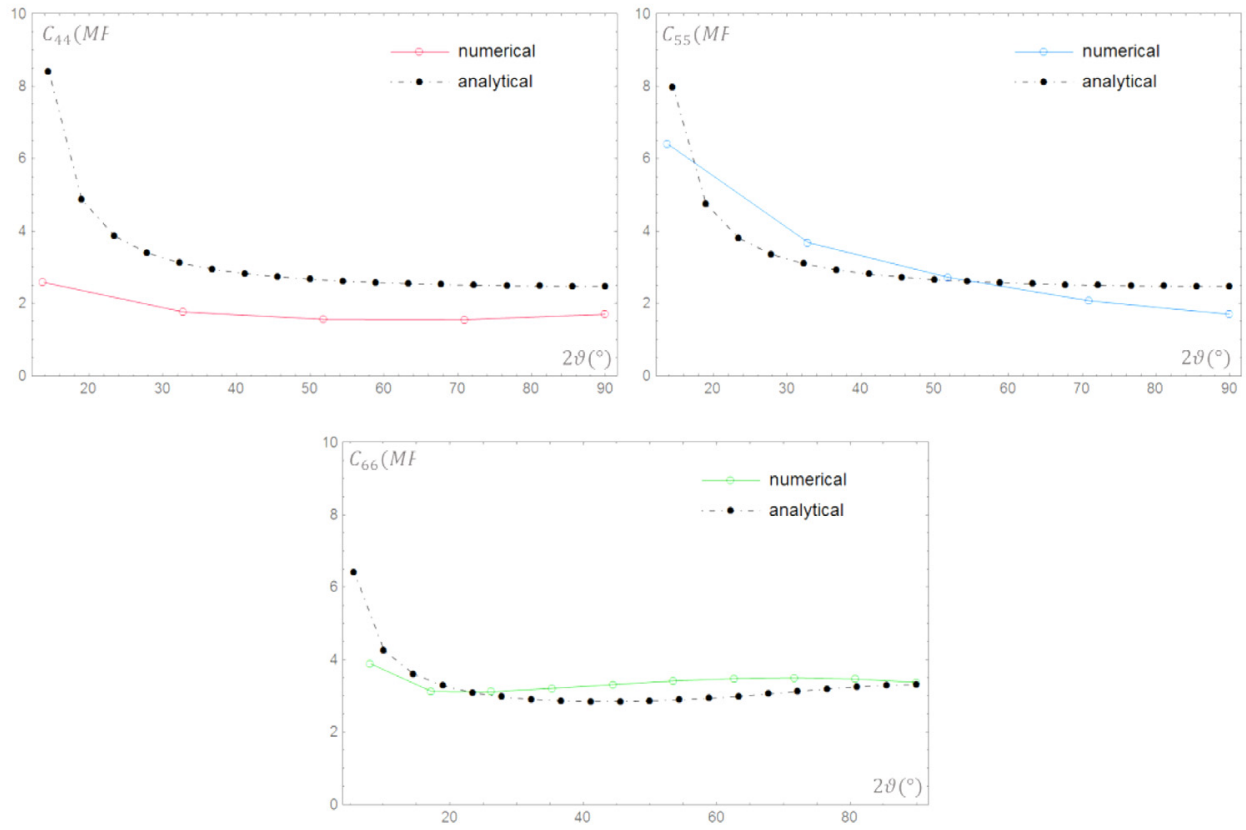

c: Compared numerical and analytical simulations of estimated evolutions with layer misorientation angle $2 \theta$ of the lateral xl-x3 (top, left), the xl-x2 facial (top, right) and the in-plane $x 2-x 3$ (bottom) shear modulus at $h=0$.

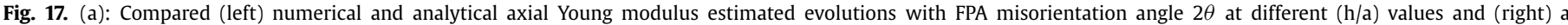

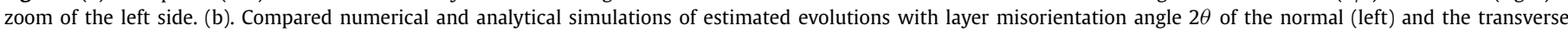

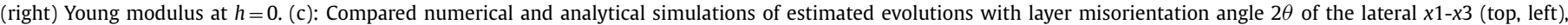
the $x 1-x 2$ facial (top, right) and the in-plane $x 2-x 3$ (bottom) shear modulus at $h=0$. 


\section{Table 7}

Data for the plots of the force-displacement numerical curves in Fig. 18. $\mathrm{h}_{\mathrm{p}}, \mathrm{r}_{\mathrm{p}}$ are the pivot heigth and radius, $\bar{\ell}$ is the inverse of the inter distance $\ell$ between pivots and $\mathrm{k}_{\mathrm{p}}=\frac{\mu \pi r_{\mathrm{p}}^{A}}{2 \mathrm{~h}_{\mathrm{p}}} \bar{\ell}^{2}$ (Spagnuolo et al., 2017).

\begin{tabular}{llllllll}
\hline$a$ & $A$ & $k_{e}=E A$ & $k_{p}^{\text {ref }}$ & $h_{p}$ & $r_{p}$ & $\bar{\ell}$ & $k_{p}^{((T))}$ \\
\hline $10^{-3} \mathrm{~m}$ & $a^{2}$ & $149.9 \mathrm{~N}$ & $1225 \cdot 10^{-5} \mathrm{~N} \mathrm{~m}^{-1}$ & $10^{-3} \mathrm{~m}$ & $5 \cdot 10^{-4} \mathrm{~m}$ & $25 \cdot 10^{-3} \mathrm{~m}^{-1}$ & $428 \cdot 10^{-5} \mathrm{~N} \mathrm{~m}^{-1}$ \\
\hline
\end{tabular}

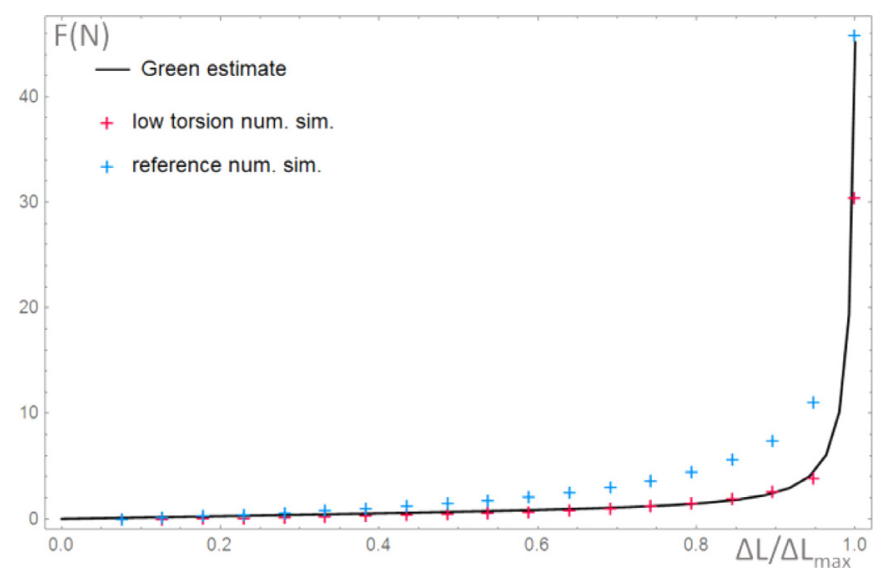

Fig. 18. Matching the analytical force-displacement curve for $h=0$ and $\mu \mathrm{M}=1$ (full line) in lowering the torsion "modulus" from the 2D pantograph (upper crosses) numerical reference curve to the "low torsion" one.

\subsubsection{Numerically matching the analytical pivot-strength-free reference curve}

The Fig. 18 reports in full lines the force-displacement curve from the analytical modeling that corresponds to the case $(h=0$, $\mu M=1)$ plotted in Fig. 15 right, together with two numerical curves that correspond to a 2D pantograph under in-plane extension, the parameters of which are collected in Table 7. The analytical curve is called the "pivot-strength-free reference" owing to the quite high phase shear modulus contrast of 50 it results from and although decreasing further the matrix shear modulus for an increased contrast can still decrease the stiffness response of the P-I composite. We call the stiffest of the two numerical curves, the "reference numerical simulation" curve (the torsion "modulus" of which is denoted "ref" in Table 5) and the second one, which is the numerical adjustment on the analytical curve is called the "low torsion numerical simulation" curve ("IT" in Table 7). The pretty good adjustment is obtained from reducing the pantograph (pivot) torsion modulus by a factor 3 from the value of the reference numerical curve. A lower analytical reference curve could similarly be adjusted in lowering the pantograph torsion modulus by a larger factor. For one discussion point to come next on, it is worth to stress that adjusting analytical curves obtained with a smaller $\mu M$ matrix modulus (keeping same $h$ value) would be reasonably well obtained in decreasing similarly further the pantograph torsion modulus in the numerical simulation, such that a no torsion strength limit in the pantograph extension numerical simulation expectedly calls for a vanishing matrix stiffness in the proposed analytical modeling.

\subsubsection{Analytically matching the numerical reference curve with adding pivot strength effects}

Considering first the pivot strength effects to be purely torsional, a first possible input in the proposed modeling is on the pivot effective stiffness that explicitly appears in the description given to the bundle-like pivot array that reinforces the embedding matrix according to Eq. (24). A pivot stiffness increase can be accounted for in giving some evolution to their shear modulus with the misorientation angle between fiber layers. A qualitative evi- dence that alternated torsion of successive pivots along fiber-like arrays is unavoidable, although hard to observed either experimentally or numerically, can be inferred from imagining the extension of the multi-layered P-I structures exemplified in Fig. 1. In order to check this effect, we have considered an evolution law of the bundle shear stiffness, under the simple form:

$\mu_{B}(\theta / h)=\mu_{B}\left(1+c\left(\left|\frac{\pi}{4}-\theta\right| \frac{b}{h+b}\right)\right)^{N}$

where " $c$ " and the exponent $N$ are adjustable coefficients, while the absolute value results from assumed equal stiffness increase whether the $\theta$ angle decreases or increases from the unstressed $\pi / 4$ position.

In order to next examine a bending interpretation of the pivot strength effects in the sense of an inclination with regard to the normal to the FPAs, a simple possibility to introduce it in the analytical modeling is to consider that the main pivot bending effect is to reduce the distance $(h)$ between the FPA layers, with at the most, layers reaching contact if that bending goes to its geometrical limit (without damage). In contrast with pivot torsion this alternated bending of pivots along fiber-like arrays has established existence as exemplified on Fig. 19 left, from some performed experiments not to be commented in the present scope. It is also observable that the assumption of this (not totally regular) alternated bending to be null in the average over the layers is reasonable in gross approximation and sufficient for a schematic description of the layer inter distance decrease as shown in Fig. 19 right. The bending is assumed to remain in the plane made by the extension direction and the pivot array initial orientation normal to the FPA layers (the drawing plane) although each fiberlike pivot array connect fibers which are alternately $\pm \theta$ misoriented on each side of it. The inclination angle can be estimated from the projections of the FPA layers in that "bending plane" as defined and drawn. A pivot bending of (mean) angle $\pm \kappa=( \pm \boldsymbol{w}, \boldsymbol{z})$, with $\pm \boldsymbol{w}$ and $\boldsymbol{z}$ being respectively the pivot (alternated) direction and the FPA normal, typically changes the FPA inter distance from $h$ to $h(\kappa)=(h+b) \cdot \cos (\kappa)-b$. The limit bending angle $\kappa_{\text {lim }}$ at layer contact $(h=0)$ may be restricted by some minimal $h_{\min }$ value corresponding to a $\kappa_{\max }$ angle smaller than $\kappa_{\text {lim }}$, in which case $h^{\prime}\left(\kappa_{\max }\right)=(h+b) \cdot \cos \left(\kappa_{\max }\right)-b=h_{\min }$ and $\kappa_{\max }=\operatorname{arcos}\left(\frac{h_{\min }+b}{h+b}\right)$. Note that in comparison with the possibly significant decrease of $h$ if approaching or reaching contact $\left(h_{\min }=0, \kappa_{\max }=\kappa_{\text {lim }}\right)$, the pivot bending contribution to the structure in the extension direction is a negligible axial translation of the left-oriented FPAs with regard to the right-oriented ones, of value $(h h+b) \cdot \sin \left(\kappa_{\max }\right)$. The introduction in the modeling of this pivot bending (inclination) effect on $(h)$ can then pass by Eq. (30) to increase faster the fiber volume fraction as:

$f_{f i b(h, \kappa)}=\left(\frac{b}{h(\kappa)+b}\right) \frac{a}{L \sin 2 \theta}=\left(\frac{b}{h(\kappa)+b}\right) f_{f i b(0)}$.

The use of Eq. (33) needs to determine or to fix an evolution of $\kappa / \kappa_{\max }$ in terms of $\theta / \theta_{\max }$ or of any related "geometric descriptor" as defined. For the present purpose, only variations of $F / \Delta L$ curve with different constant $h / a$ values have been compared.

These two pivot strength effects are exemplified in Fig. 20 left for the pivot stiffness (shear modulus) variation according to Eq. (32) and in Fig. 20 right for the inclination stiffness effect from 

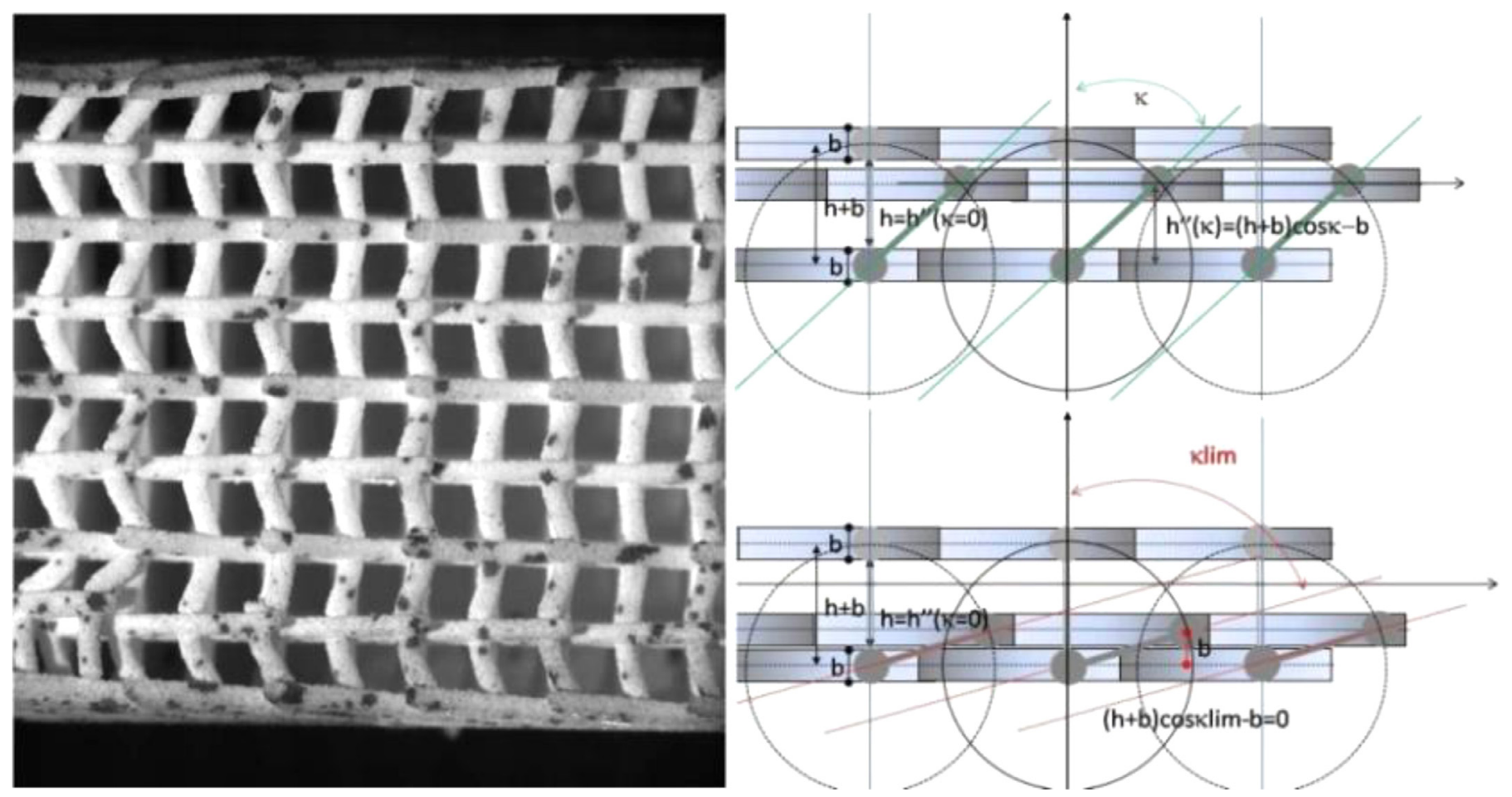

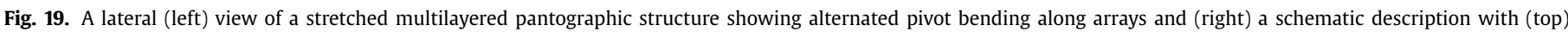

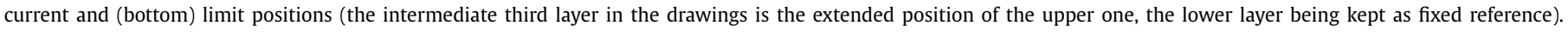
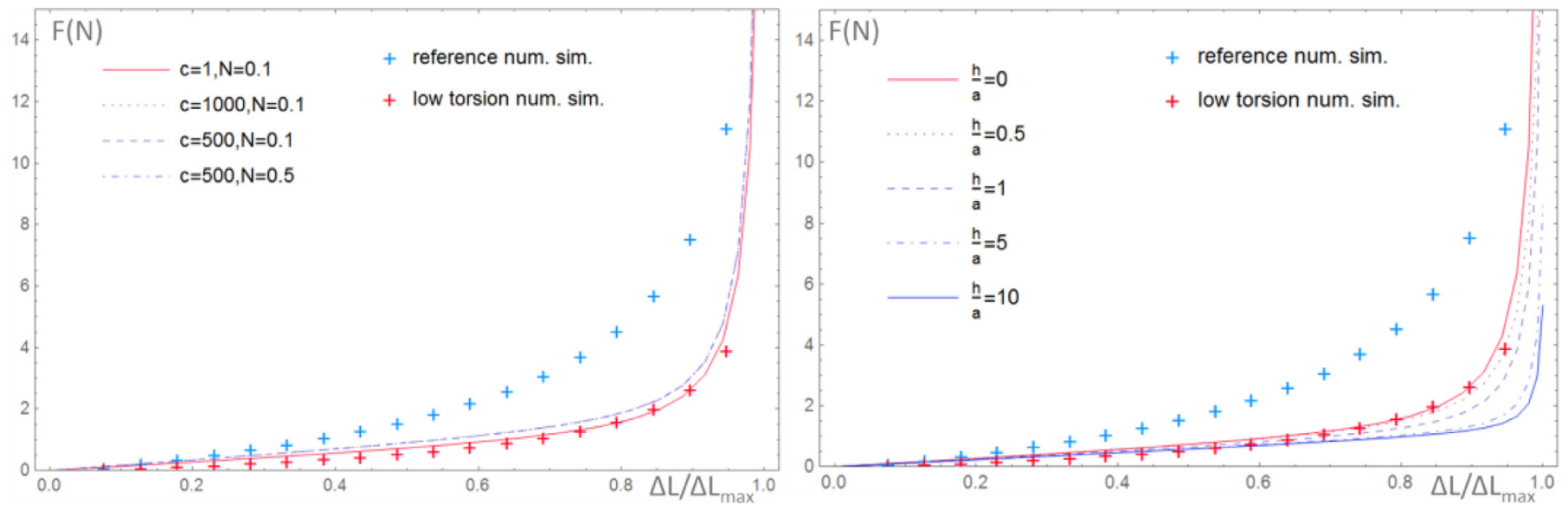

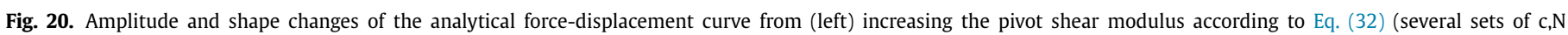
parameters) and (right) decreasing the h/a inter distance.

changing the (kept constant) value of the layer inter distance $(h / a)$, without using Eq. (33). It is noteworthy regarding the pivot height changes due to bending that a division by two of $h$ may be likely corresponding to the transition amounts from 10 to 5 or from 1 to 0.5 which are exemplified in Fig. 20 right.

From these Figures, where both the pantograph reference numerical curve and the low torsion one are plotted, the amount of variation allowed on a force-displacement curve from these two effects, either alone or together, both on the amplitude and on the shape of the curve only appears convenient to adjust weak or moderate pivot strength effects.

On the contrary, when the contribution of pivot strength effects to the total curve is more important, as is the case for the numerical reference curve in Figs. 18 and 20, these two effects are not sufficient. In which case, a last possibility to indirectly consider a pivot-due effect of torsion-type in the proposed analytical modeling is through a dependency of the matrix stiffness with the misorientation angle $2 \theta$ between the fiber layers. The Fig. 21 left reports again the pantograph reference numerical curve with a set of analytical ones for different phase contrasts $\mu M / \mu F$ at constant $\mu F$ value, starting from $\mu M=0.02 \mu \mathrm{F}$ (which is the contrast with $\mu M=1$ used in most of the previous analytical plots) to end with $\mu M=0,98 \mu \mathrm{F}$. Collecting the intersections of these analytical curves with the reference numerical one yield a $\mu M / \mu F$ versus displacement $\Delta L / L \max$ relation which in turn yields the $\mu M / \mu F$ versus $2 \theta$ angle variation law plotted in Fig. 21 right. This shows to finish that using in the proposed homogenization-based modeling of PI composites a well parametrized analytical expression of such a function (as the exemplified dotted curve, the empirical formula of which is of no interest here) is capable to quite well restitute analytically any pantograph representative numerical curve.

This last figure interestingly suggests that a pantographic-like behavior of the considered P-I composite more likely correspond to a matrix with increasing stiffness than to a constant one, with a 


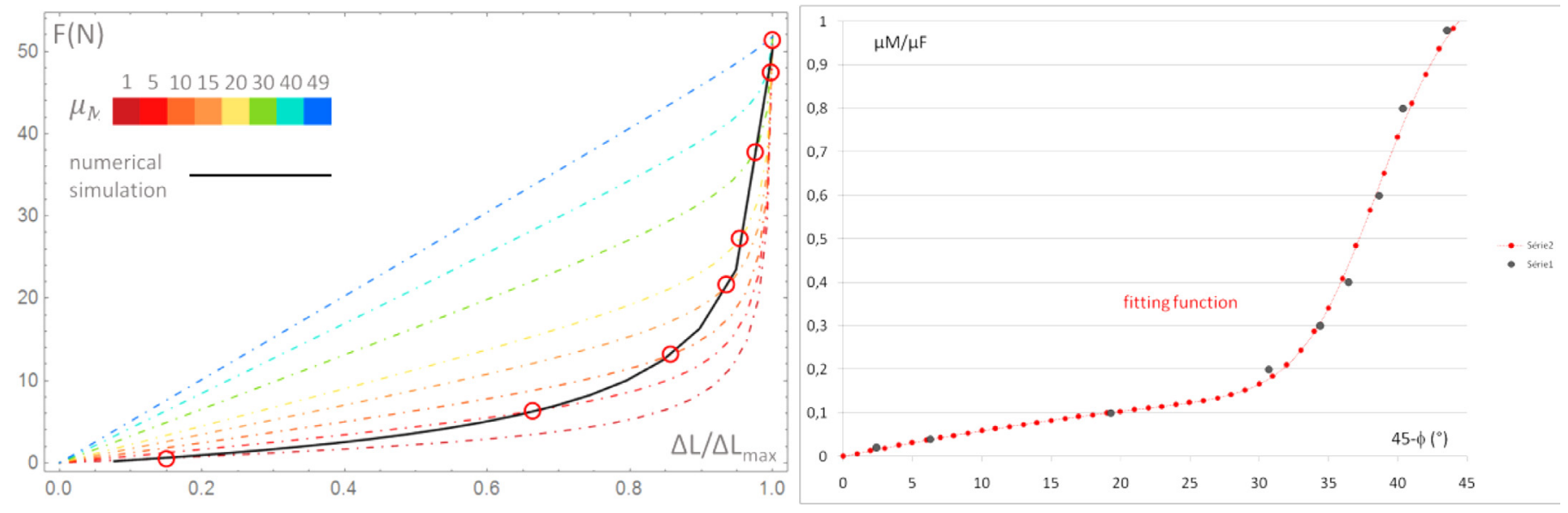

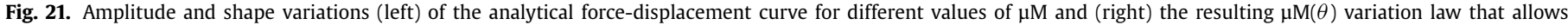
analytical matching with the reference numerical plot.

phase contrast variation of course bounded by above at $\mu \mathrm{F} / \mu \mathrm{M}=1$ (the lower bound being zero in reference to the matrix-free fiber structure), an upper value that is not expected to be reached if preserving the pantograph large deformation potential at low energy cost is one main objective of realizing such composites. The marked stiffness contrast between the fiber network phase and the matrix one in the sense of a "softer matrix" as was specified will certainly remain an essential criterion for manufacturing such PI composites. Defining "bounds" narrower than 0 and 1 for this $\mathrm{M} / \mathrm{F}$ contrast would depend on other requests on the desired composite behavior than limiting the necessary energy for deforming the pantographic network, as in particular damage considerations that we did not enter at all in this presentation. The comparison of the role of the fiber layer connectors (the pivots) in the 2D pantographs or in the multilayered ones (say when no matrix is embedding) and of the role of the matrix in the proposed and examined P-I composite type (where no physical connectors are so far integrated) is a major issue for further studies on this structure type and on its potentialities. Whether the fiber layers need, within the composite, that is within the matrix, be additionally physically connected by pivot-like elements, the answer is likely positive for if the matrix stiffness here seems to some extent play a role similar to the role of the pivot strength (on the strengthening of the structure when expending it), the pivots and the matrix are not expected to be equivalent in all mechanical aspects.

It is noteworthy that these brief temporary conclusions on so called pivot strength effects on the one hand and on matrix strengthening effects on the other hand are based on several guesses on how a real P-I composite of the here imagined type would really behave under extension. The here reported conclusive considerations do not relieve further investigations on such PI composites still needing be elaborated, with and without inter connections between the pantographic-like alternated fiber layers and with matrices of different behavior law for comparisons and future developments.

\section{Conclusion}

In this work we have presented a description for two phase bi-continuous composites made of a 3D continuous fiber network embedded in a compliant matrix, the main characteristic of which is to obey a pantographic-inspired (P-I) behavior owing to the pantographic-like nature of the fiber network deformation potentialities at low energy expenses. For such a P-I composite which is described from considering the fiber network as an alternated piling of fiber planar alignments (FPAs) whose elements are ei- ther cylindrical rods or square beams, a homogenization-based modeling is proposed for the elastic behavior, thanks to mean Green operators (mGOs) for such FPAs made available in previous works by authors and co-workers. A homogenization-based modeling amounts to estimating the effective (here elastic or elastic-like but also possibly other) properties of a composite structure in accounting as precisely as possible for its phase arrangements, especially if this arrangement evolves during deformation as is the case, such as to follow the effective property evolutions during deformation and to estimate the strain and stress-related evolutions. The characteristics of this P-I composite structure are defined from geometric descriptors which link in a 2D pantograph, on the one hand the fiber layer misorientation changes to their network extension and to the fiber inter-distances in each constitutive layer, what also modifies the fiber network volume fraction in a representative volume element of the composite. On the other hand, that same fiber layer misorientation evolution is linked to the strengthening behavior of the pivots that inter-connect the fiber layers and which constitutes a more or less important contribution to the total force-displacement curve for such fiber assemblages.

From comparisons with numerical estimates of the evolutions of the effective elastic moduli obtained for such a P-I composite structure from the proposed homogenization-based modeling, it is shown that the modeling, which has the advantage of being analytical provides, in spite of an over simplified behavior given to the matrix phase, satisfying estimates of the effective Young and shear modulus variations when such a composite is submitted, at least piece wisely, to in-plane axial homogeneous extension. Comparisons with numerically obtained force-displacement curves of an axially strained typical 2D pantograph have then shown that a quite good match is obtained with an analytically obtained forcedisplacement curve (from using the evolving effective axial Young modulus estimate) provided the adjustment of the pivot-strengthrelated contribution in the pantograph numerical extension simulation. Converse simulations have also shown that a quite good match of a numerical pantograph force-displacement curve can be obtained from the analytical modeling with accounting for (i) a torsion-due stiffness increase of the fiber-bundle like pivot arrays or/and (ii) a bending-due decrease of the fiber layers inter-distance or/and (iii), for high pivot strength effects, with assuming the embedding matrix of the composite to have a fiber misorientationdue stiffness increase during straining. These matching possibilities show that such pantographic-inspired composites can also be, to some extent, pantograph-equivalent. However, the comparison of the role of the fiber connectors (the pivots) in the 2D pantographs or the multilayered ones (say when no matrix is em- 
bedding) and of the role of the matrix in the proposed and examined pantographic-inspired composite type (where no physical connectors are so far integrated) remains a major issue for further studies on this structure type and on its potentialities. Whether fiber layers need, within such a P-I composite, be physically connected by pivot-like elements is likely for the pivots and the matrix are far from equivalent in all mechanical aspects. A 3D fiber network really co-continuous with an embedding matrix requires inter connected fiber layers. Manufacturing such P-I two-phase composites is the request for future developments and for studies of real matrix behavior when embedding such a fiber network with or without physical interconnections of the fiber layers and for the pivot strength effects within a matrix phase. Owing to the first summarized results, such P-I composites are believed promising structures in a wide range of applications.

\section{Acknowledgments}

Authors are particularly grateful to Prof. U. Andreaus (Università di Roma "La Sapienza") for allowing the use of a numerical force-displacement simulation of a 2D pantograph using a EulerBernoulli non-linear beam-based code of the model presented in Andreauset al. (2018) and to Prof. T. Lekszycki and his group (Warsaw University of Technology) for AM realization of some multilayered pantographs as the ones shown in Figs. 1 and 19.

Mario Spagnuolo has received funding from the European Union's Horizon 2020 research and innovation programme under the Marie Skłodowska-Curie grant agreement No 665850.

\section{Appendix A. angular relations for the Fig. 4 pantograph deformed axial and transverse states}

In the extension case, for a $\theta$ angle in the central zone given by the extension amount, identically the strained four lateral zones correspond to a $\theta^{\prime}$ angle equal to $0.5(\theta+\pi / 4)$. The in-plane bending angle of the two fiber families is simply $\beta=\pi / 4-\theta$. In the shear case, corresponding to a $D$ transverse displacement of point $\mathrm{P}$ with regard to point $\mathrm{R}$ (and no extension), one has $\gamma=D / \operatorname{Lsqrt}(2)$ and the solid body rotation is $r=\operatorname{atan}(\gamma)$. The $\theta$ angle for the central zone is given from $\cos \theta=\operatorname{sqrt}\left(\left(1+\gamma^{2}\right) / 2\right)=\operatorname{sqrt}\left(\left(1+\tan ^{2}(\mathrm{r})\right) / 2\right)$. When $\gamma$ or $\mathrm{r}$ is zero, there is no deformation and $\cos \theta=1 / \operatorname{sqrt}(2)=\cos \pi / 4$. The two types of lateral zones correspond to an angle $0.5(\theta+\pi / 4)+/-\mathrm{r}$ and the two in-plane bending angles for the fiber families are $\pi / 4-\theta$ $+/-r$, that is in both case the extension values with adding or substracting the rigid body rotation. A more general in-plane deformation combining extension and shear can be analyzed similarly. Pantographs with more zones than a single central one between two triangular ends and four lateral parts can be schematized in similar yet more complicated manner.

\section{Appendix B. The form of the elementary operators $t^{\boldsymbol{e}}$ for isotropic elasticity}

This brief summary is extracted from Franciosi and Lormand (2004) and Franciosi (2005).

Each $\boldsymbol{t}^{e}(\boldsymbol{\omega})=\boldsymbol{t}^{e}(\theta, \phi)$ elementary operator in $\boldsymbol{t}^{V}(\boldsymbol{r})$ from Eq. (8) is an axi-symmetric operator, defined in reference to the parallel planes of $\boldsymbol{\omega}$ normal direction in some reference medium frame. For general elasticity anisotropy, considering the $(0,0)$-oriented $\boldsymbol{t}^{e}(0,0)$ elementary operator for $(\theta, \phi)=(0,0)$, the only non zero terms from Eq. (7c) correspond to $\omega=(0,0,1)$ and the $M_{m p}$ coefficients identify to the $C_{m 3 p 3}$ elastic moduli of the infinite medium, for $\boldsymbol{C}$ expressed in the operator axes frame as $\boldsymbol{C}(0,0)$. In this frame, $\boldsymbol{M}$ is a $3 \times 3$ symmetric sub-matrix of $\boldsymbol{C}(0,0)$ and the $t_{p 3 j 3}^{e}(0,0)$ non zero terms of the $\boldsymbol{t}^{e}(0,0)$ operator expressed in the operator frame
Table B

Non zero iijj (top) and ijij (bottom) terms of the elementary operator $\boldsymbol{t}^{e}(\theta, \phi)$, with " $\mathrm{c} \theta$ " and "s $\theta$ " for " $\cos \theta$ "and " $\sin \theta$ " (resp. $\phi$ ).

\begin{tabular}{llll}
\hline & 11 & 22 & 33 \\
\hline 11 & $\mathrm{As}^{4} \theta \mathrm{c}^{4} \phi$ & $\mathrm{As}^{4} \theta \mathrm{c}^{2} \phi \mathrm{s}^{2} \phi$ & $\mathrm{As}^{2} \theta \mathrm{c}^{2} \theta \mathrm{c}^{2} \phi$ \\
& $\mathrm{Bs}^{2} \theta \mathrm{c}^{2} \phi$ & 0 & 0 \\
22 & $\mathrm{As}^{4} \theta \mathrm{c}^{2} \phi \mathrm{s}^{2} \phi$ & $\mathrm{A} \mathrm{s}^{4} \theta \mathrm{s}^{4} \phi$ & $\mathrm{As}^{2} \theta \mathrm{c}^{2} \theta \mathrm{s}^{2} \phi$ \\
33 & 0 & $\mathrm{Bs}^{2} \theta \mathrm{s}^{2} \phi$ & 0 \\
& $\mathrm{As}^{2} \theta \mathrm{c}^{2} \theta \mathrm{c}^{2} \phi$ & $\mathrm{As}^{2} \theta \mathrm{c}^{2} \theta \mathrm{s}^{2} \phi$ & $\mathrm{Ac}^{4} \theta$ \\
2323 & 0 & 0 & $\mathrm{Bc}^{2} \theta$ \\
$\mathrm{As}^{2} \theta \mathrm{c}^{2} \theta \mathrm{s}^{2} \phi$ & 3131 & 1212 & \\
$\mathrm{~B}\left(\mathrm{~s}^{2} \theta \mathrm{s}^{2} \phi+\mathrm{c}^{2} \theta\right) / 4$ & $\mathrm{~A} \mathrm{~s}^{2} \theta \mathrm{c}^{2} \theta \mathrm{c}^{2} \phi$ & $\mathrm{A} \mathrm{s}^{4} \theta \mathrm{c}^{2} \phi \mathrm{s}^{2} \phi$ & \\
\hline
\end{tabular}

make a symmetric $3 \times 3$ matrix as well, $\Delta \boldsymbol{t}$ say, such that $t_{p 3 j 3}^{e}=$ $\Delta t_{p j}=M^{-1}{ }_{p j}$. Appropriate tensor rotations give the $\boldsymbol{C}(\theta, \phi)$ tensors and sub-parts as well as the related operators $\boldsymbol{t}^{e}(\theta, \phi)$ as further detailed in Franciosi and Lormand (2004).

For elastic isotropy, since in all frames $C_{3333}=\lambda+2 \mu=$ $2 \mu(1-v) /(1-2 v)$ and $C_{1313}=C_{2323}=\mu$, the $\boldsymbol{C}$ frame identification is made useless. The non zero terms of $\Delta t$ are $\Delta t_{11}=1 / C_{1313}$, $\Delta t_{22}=1 / C_{2323}, \Delta t_{33}=1 / C_{3333}$ and those of $\boldsymbol{t}^{e}(\theta, \phi)$ are $t_{((2,3),(2,3))}=$ $t_{((3,1),(3,1))}=\frac{1}{4 \mu}=\frac{B}{4}$ and $t_{3333}=\frac{1-2 \nu}{2 \mu(1-\nu)}=\frac{1}{\mu}-\frac{1}{2 \mu(1-v)}=B+A$, for all $\forall(\theta, \phi) \equiv \boldsymbol{\omega}$. The $\boldsymbol{t}^{e}(\theta, \phi)=\boldsymbol{t}^{e}(\boldsymbol{\omega})$ operator terms thus rearrange as $t_{p q j n}^{e}(\boldsymbol{\omega})=A \tau_{p q j n}^{A}(\omega)+B \tau_{p q j n}^{B}(\boldsymbol{\omega})$ with $\tau_{p q j n}^{A}(\boldsymbol{\omega})=$ $\omega_{j} \omega_{p} \omega_{n} \omega_{q}$ and $\tau_{p q j n}^{B}(\boldsymbol{\omega})=\left.\left(\delta_{j p} \omega_{n} \omega_{q}\right)\right|_{(p, q),(j, n)}$. They are given in Table B from Franciosi (2005)

These non zero terms are defined by even trigonometric functions of the form $f^{(l, m)}(\theta) g^{(r, s)}(\phi)=\cos ^{2 l} \theta \sin ^{2 m} \theta \cos ^{2 r} \phi \sin ^{2 s} \phi$, which are the non vanishing ones upon integration over the unit sphere when multiplied by any positive and even function in $(\theta, \phi)$ as the shape function $\psi_{V}(\theta, \phi)$ is in Eq. (8). Owing to the five (( 1 , $0),(0,1))$ and $((2,0),(1,1),(0,2))$ possible values taken by both the $(1, \mathrm{~m})$ and $(\mathrm{r}, \mathrm{s})$ exponent pairs, owing to the dependency relations between the trigonometric functions of concern, all terms can be expressed in using only two of the five $f^{(l, m)}(\theta)$ functions, one in each exponent pair set within brackets, and similarly two of the five $g^{(r, s)}(\phi)$ ones, such as for example $(l, m)=(1,0),(2,0)$ and $(r$, $s)=(1,0),(2,0)$ which respectively correspond to the two functions $\cos ^{2}(\iota)$ and $\cos ^{4}(\iota)$ for each angle $\iota=\theta, \phi$. Thus from Eq. (8), the mGO terms over some domain $V$ read $\overline{t_{p q j n}^{V}}=\int_{\Omega} t_{p q j n}^{e}(\omega) \overline{\psi_{V}(\boldsymbol{\omega})} d \omega$ and explicating the elementary (iotropic elastic) operator part in it, the integrals which then need to be calculated are all of the form:

$\overline{t_{p q j n}^{V}}=\int_{\theta=0}^{\pi} \int_{\varphi=0}^{2 \pi} \overline{\psi_{V}(\theta, \varphi)}\left(\cos ^{2 l} \theta \sin ^{2 m} \theta \cos ^{2 r} \varphi \sin ^{2 s} \varphi\right) \sin \theta d \theta d \varphi$

and for the selected ones, with $l,=1,2$ and $r=1,2$ (each corresponding to some terms in Table B):

$\int_{\theta=0}^{\pi} \int_{\varphi=0}^{2 \pi} \overline{\psi_{V}(\theta, \varphi)}\left(\cos ^{2 l} \theta \cos ^{2 r} \varphi\right) \sin \theta d \theta d \varphi$

Depending on whether the two integrals are independent or not and according to the pattern geometry description, that is depending on the shape function characteristics, one of the two integrals can be simplified such that only two remain to be calculated, which can be generically denoted $C 2$ and $C 4$. In the particular cases of patterns with infinite parallel fibers all oriented in the $x 2$ direction, the shape function is only non zero "around" the fiber direction, which is the $x 1-x 3$ plane in which the angle $\phi$ is null such that Eq. (B2) becomes a pair of $\theta$ integral for $l=1,2$ times $2 \pi$ from the $\phi$ one as:

$2 \pi \int_{\theta=0}^{\pi} \overline{\psi_{V}(\theta, 0)}\left(\cos ^{2 l} \theta\right) \sin \theta d \theta$ 
This introduces the two $\mathrm{C} 2 \mathrm{l}$ functions for the mGO of C-fibers and SQ-beams FPAs recalled in Section 2, with explicated further details in Franciosi et al. (2019) and Franciosi (2018).

\section{Appendix C. some details of a bi-layer effective properties from the laminate description}

As is shown in Table $C$, the non zero terms of $\Delta \boldsymbol{C}_{e f f P C W}^{x 1 F P A( \pm \theta)}=$ $\pm 2 \boldsymbol{C}^{S S(\theta)}$ in EqS. (19) and (20) are $\Delta C_{X 11,(2,3)}^{X 1 F P A( \pm \theta)}, \Delta C_{X 22,(2,3)}^{X 1 F P A( \pm \theta)}$, $\Delta C_{X 33,(2,3)}^{X 1 F P A( \pm \theta)}, \Delta C_{X(1,2),(1,3)}^{X 1 F P A( \pm \theta)}$ and the non zero terms of the laminate operator $\boldsymbol{t}_{\{C\}}^{p x_{1}}=\boldsymbol{t}_{\left\{\boldsymbol{C}_{e f f P C W}^{R B( \pm \theta), x_{1}}\right\}}^{p x_{1}}$ read (with $c=\cos (\theta), s=\sin (\theta)$, $\bar{T}=1 / T)$ :

$t_{\{C\} 1111}^{p x 1}=\overline{\{C\}_{1111}^{O(\theta)}}=\overline{C_{1111}^{(0)}}$,

$t_{\{C\} 1313}^{p x 1}=\overline{\{C\}_{1313}^{O(\theta)}}=\overline{c^{2} \mu_{1313}^{(0)}+s^{2} \mu_{1212}^{(0)}}$

$t_{\{C\} 1212}^{p \times 1}=\overline{\{C\}_{1212}^{O(\theta)}}=\overline{s^{2} \mu_{1313}^{(0)}+c^{2} \mu_{1212}^{(0)}}$

In Eqs. (C1), $\boldsymbol{C}^{(0)}=\boldsymbol{C}_{\text {effPCW }}^{x 1 F P A(0)}$ represents the effective stiffness property tensor chosen for the generic layer prior to $\mathrm{a} \pm \theta$ rotation, where $\mu_{i j i j}^{(0)}$ stand for the shear moduli. The terms of $\boldsymbol{C}_{\text {effLS }}^{x 1 P A N T( \pm \theta)}$ in Eq. (19) which have a non-zero second part are $C_{e f f L S(1,2),(1,2)}^{x 1 P A N T( \pm \theta)}$ and $C_{\text {effLS }}^{x 1 P A N T( \pm),(1,3)}$, due to $\left(\Delta C_{X(1,2),(1,3)}^{x 1 F P A( \pm)}\right)^{2}$ multiplied by $t_{\{C\} 1313}^{p x 1}$ and $t_{\{C\} 1212}^{p x 1}$ respectively, and $C_{e f f L S(2,3),(2,3)}^{x 1 P A N T( \pm \theta)}$ due to $\left(\Delta C_{X 11,(2,3)}^{x 1 F P A( \pm \theta)}\right)^{2} \mathrm{mul}-$ tiplied by $t_{\{C\} 1111}^{p \times 1}$.

It is seen that the alternated orientations of the fiber reinforcements in the layers make the three effective shear moduli of the laminate structure to differ from the Voigt estimate, with differences resulting from coupling effects: the additional term for the in-layer plane shear component $((2,3),(2,3))$ are induced by the laminate operator term (1111) normal to the layers and the additional term in the out-of-plane shear components $((1,2),(1,2))$ (resp. $((1,3),(1,3)))$ resulting from the shear laminate operator term $((1,3),(1,3))$ (resp. $((1,2),(1,2)))$.

The amplitude of the deviation from the Voigt estimate is maximum when the two fiber layers alternate orthogonally $(\theta= \pm \pi / 4)$ since all the skew-symmetric terms of $(\Delta \boldsymbol{C})^{2}$ are proportional to $\sin ^{2} \theta \cos ^{2} \theta$ and it also varies with the anisotropy of $\boldsymbol{C}^{(0)}$ since $\Delta C_{X 11,(2,3)}^{X 1 F P A( \pm \theta)}\left(\right.$ resp. $\Delta C_{X(1,2),(1,3)}^{X 1 F P A( \pm \theta)}$ ) cancels with $C_{11,22}^{c f i b(0)}-C_{11,33}^{C f i b(0)}$ (resp. with $\left.C_{(1,2),(1,2)}^{c f i b(0)}-C_{(3,1),(3,1)}^{c f i b(0)}\right)$.

\section{Appendix D. Prescribed boundary condition for numerical} simulations on the cell of Fig. 16

For validating the presented analytical estimates of the elastic moduli of the pantographic-inspired composite, FEM numerical simulations have been performed by considering a 3D cell whose sides are provided of periodic boundary conditions for allowing the identification with the infinite case. The representative "volume" element (RVE), shown in the reference and deformed configuration in Fig. 16, consists of three squared beams alignments with an initial relative misorientation of $90^{\circ}$. The three alignments are embedded in a parallelepiped box form representing the matrix phase and they are lying on three parallel planes whose inderdistance represents the $h$ parameter of the analytical model. Each deformed shape corresponds to a specific value of the misorientation angle 29. The Meshing type is exemplified in Fig. D left. We derive the numerical estimations of the Young $E_{1}, E_{2}$ and $E_{3}$ and shear $C_{44}$, $C_{55}$ and $C_{66}$ moduli.

Boundary conditions are given on the displacement vector $\boldsymbol{u}=$ $(u, v, w) \in \mathbb{R}^{3}$, according to the nomenclature of the cell sides defined in Fig. D right. The displacements on side $A B$ are called $\boldsymbol{u} A B=$ $(u A B, v A B)$ and similarly for the other sides. For computing the Young moduli $E_{1}, E_{2}$ and $E_{3}$, three infinitesimal compressions along the three axes have been performed. The prescribed boundary con$u\left(\hat{x}_{1}^{+}\right)=-u\left(\hat{x}_{1}^{-}\right)$
ditions are $\left\{v\left(\hat{x}_{2}^{+}\right)=-v\left(\hat{x}_{2}^{-}\right)\right.$
$w\left(\hat{x}_{3}^{+}\right)=w\left(\hat{x}_{3}^{-}\right)$

The three compressions need the same boundary conditions to simulate an infinite-like structure. Appropriate boundary conditions are needed for determining the shear moduli:

$$
\begin{aligned}
& \text { for } C_{44}\left\{\begin{array} { l } 
{ w ( \hat { x } _ { 1 } ^ { + } ) = w ( \hat { x } _ { 1 } ^ { - } ) } \\
{ w ( \hat { x } _ { 2 } ^ { + } ) = - w ( \hat { x } _ { 2 } ^ { - } ) , \text { for } C _ { 5 5 } } \\
{ w ( \hat { x } _ { 3 } ^ { + } ) = w ( \hat { x } _ { 3 } ^ { - } ) }
\end{array} \left\{\begin{array}{l}
w\left(\hat{x}_{1}^{+}\right)=-w\left(\hat{x}_{1}^{-}\right) \\
w\left(\hat{x}_{2}^{+}\right)=w\left(\hat{x}_{2}^{-}\right), \text {for } \\
w\left(\hat{x}_{3}^{+}\right)=w\left(\hat{x}_{3}^{-}\right)
\end{array}\right.\right. \\
& C_{66}\left\{\begin{array}{l}
v\left(\hat{x}_{1}^{+}\right)=-v\left(\hat{x}_{1}^{-}\right) \\
v\left(\hat{x}_{2}^{+}\right)=v\left(\hat{x}_{2}^{-}\right) \\
v\left(\hat{x}_{3}^{+}\right)=v\left(\hat{x}_{3}^{-}\right)
\end{array}\right.
\end{aligned}
$$

The requested moduli are numerically calculated via the analogous of an experimental measure, imposing an external small displacement $\boldsymbol{u}_{0}$ to one of the sides and calculating the desired modulus as ratio between the computed stress and the global strain.

Table C

Non zero even (top) and odd (bottom) terms of the effective property tensor $\mathrm{C}$ and of the mean GO $\mathrm{t}$ for the

\begin{tabular}{|c|c|c|}
\hline Even terms & $C_{i j k l}^{F( \pm \theta)}$ & $t_{i j k l}^{F( \pm \theta)}$ \\
\hline $\begin{array}{l}1111 \\
11,22 \\
11,33 \\
2222 \\
22,33 \\
3333 \\
(2,3),(2,3) \\
(3,1),(3,1) \\
(1,2),(1,2)\end{array}$ & $\begin{array}{l}C_{1111}^{F(0)} \\
c^{2} C_{1,2)}^{F(0)}+s^{2} C_{11,33}^{F(0)} \\
s^{2} C_{11,22}^{F(0)}+c^{2} C_{11,33}^{F(0)} \\
c^{4} C_{2222}^{F(0)}+4 c^{2} s^{2} C_{(2,3),(2,3)}^{F(0)}+s^{4} C_{3333}^{F(0)}+2 c^{2} s^{2} C_{22,33}^{F(0)} \\
c^{2} s^{2} C_{2222}^{F(0)}-4 c^{2} s^{2} C_{(2,3),(2,3)}+c^{2} s^{2} C_{3333}^{F(0)}+\left(c^{4}+s^{4}\right) C_{22,33}^{F(0)} \\
s^{4} C_{2222}^{F(0)}+4 c^{2} s^{2} C_{(2,3),(2,3)}^{F(0)}+c^{4} C_{3333}^{F(0)}+2 c^{2} s^{2} C_{22,33}^{F(0)} \\
c^{2} s^{2} C_{2222}^{F(0)}+\left(c^{2}-s^{2}\right) C_{(2,3),(2,3)}^{(0)}+c^{2} s^{2} C_{3333}^{F(0)}-2 c^{2} s^{2} C_{22,33}^{F(0)} \\
c^{2} C_{(3,1),(3,1)}^{F(0)}+s^{2} C_{(1,2),(1,2)}^{F(0)} \\
s^{2} C_{(3,1),(3,1)}^{F(0)}+c^{2} C_{(1,2),(1,2)}^{F(0)}\end{array}$ & $\begin{array}{l}t_{1111}^{F(0)} \\
c^{2} t_{11,22}^{F(0)} \\
s^{2} t_{1,22}^{F(0)} \\
c^{4} t_{2222}^{F(2)}+4 c^{2} s^{2} t_{(2,3),(2,3)}^{F(0)} \\
c^{2} s^{2} t_{2222}^{F(0)}-4 c^{2} s^{2} t_{(2,3),(2,3)}^{F(0)} \\
s^{4} t_{2222}^{F(0)}+4 c^{2} s^{2} t_{(2,3),(2,3)}^{F(2)} \\
c^{2} s^{2} t_{2222}^{F(0)}+\left(c^{2}-s^{2}\right) t_{(2,3),(2,3)}^{F(0)} \\
c^{2} t_{(3,1),(3,1)}^{F(0)}+s^{2} t_{(1,2),(1,2)}^{F(0)} \\
s^{2} t_{(3,1),(3,1)}^{F(0)}+c^{2} t_{(1,2),(1,2)}^{F(0)}\end{array}$ \\
\hline \multicolumn{3}{|l|}{ Odd terms } \\
\hline $\begin{array}{l}11,(2,3) \\
22,(2,3) \\
33,(2,3) \\
(1,2),(1,3)\end{array}$ & $\begin{array}{l}\mp s c C_{11,22}^{F(0)} \pm s C C_{11,33}^{F(0)} \\
\mp s C\left(c^{2} C_{2222}^{F(0)}+2\left(s^{2}-c^{2}\right) C_{(2,3),(2,3)}^{F(0)} \pm s c\left(s^{2} C_{3333}^{F(0)}-\left(s^{2}-c^{2}\right) C_{22,33}^{F(0)}\right)\right. \\
\mp S C\left(s^{2} C_{2222}^{F(0)}-2\left(s^{2}-c^{2}\right) C_{(2,3),(2,3)}^{F(0)}\right) \pm s c\left(c^{2} C_{3333}^{F(0)}+\left(s^{2}-c^{2}\right) C_{22,33}^{F(0)}\right) \\
\mp S C\left(C_{(1,2),(1,2)}^{F(0)}-C_{(3,1),(3,1)}^{F(0)}\right)\end{array}$ & $\begin{array}{l}\mp s c t_{1,22}^{F(0)} \\
\mp s c\left(c^{2} t_{2222}^{F(0)}+2\left(s^{2}-c^{2}\right) t_{(2,3),(2,3)}^{F(0)}\right) \\
\mp S C\left(s^{2} t_{2222}^{F(0)}-2\left(s^{2}-c^{2}\right) t_{(2,3),(2,3)}^{F(0)}\right) \\
\mp S C\left(t_{(1,2),(1,2)}^{F(0)}-t_{(3,1),(3,1)}^{F(0)}\right)\end{array}$ \\
\hline
\end{tabular}
pantographic-inspired bilayer ("F" stands for C-fibers, SQ-beams and others). 

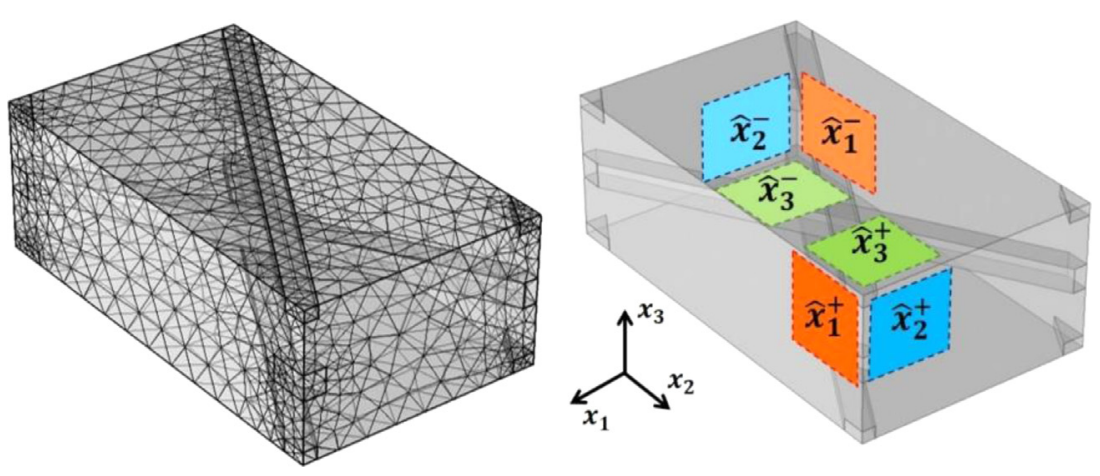

Fig. D. Numerical calculation mesh (left) and nomenclature for setting boundary conditions (right).

\section{References}

Alibert, J.J., Seppecher, P., dell'Isola, F., 2003. Truss modular beams with deformation energy depending on higher displacement gradients. Math. Mech. Solids 8 (1), 51-73.

Andreaus, U., Spagnuolo, M., Lekszycki, T., Eugster, S.R., 2018. A Ritz approach for the static analysis of planar pantographic structures modeled with nonlinear Euler-Bernoulli beams. Cont. Mech. Thermodyn. 30, 1103-1123.

Barchiesi, E., Placidi, L., 2017. A review on models for the 3D statics and 2D dynamics of pantographic fabrics. In: Wave Dynamics and Composite $\mathrm{Me}$ chanics For Microstructured Materials and Meta-Materials. Springer, Singapore, pp. 239-258.

Barchiesi, E., Laudato, M., Di Cosmo, F., 2018. Wavedispersion in non-linear pantographic beams. Mech. Res. Commun. 94, 128-132.

Barchiesi, E., Khakalo, S., 2019. Variational asymptotic homogenization of beam-like square lattice structures. Math. Mech. Solids, 1081286519843155.

Barchiesi, E., Ganzosch, G., Liebold, C., Placidi, L., Grygoruk, R., Müller, W.H., 2019. Out-of-plane buckling of pantographic fabrics in displacement-controlled shear tests: experimental results and model validation. Cont. Mech. Thermodyn. 31 (1), 33-45.

Barnett, D.M., Lothe, J., 1975. Dislocations and line charges in anisotropic piezoelectric insulators. Phys. Status Solidi B 67, 105-111.

Berveiller, M., Fassi-Fehri, O., Hihi, A., 1987. The problem of two plastic and heterogeneous inclusions in an anisotropic medium. Int. J. Eng. Sci. 25 (6), 691-709.

Bornert, M., Stolz, C., Zaoui, A., 1996. Morphologically representative pattern-based bounding in elasticity. J. Mech. Phys. Solids 44 (3), 307-331.

Boutin, C., Giorgio, I., Placidi, L., 2017. Linear pantographicsheets: asymptotic micromacro models identification. Math. Mech. Complex Syst. 5 (2), 127-162.

Buryachenko, V.A., 2001. Multiparticle effective field and related methods in micromechanics of composite materials. Appl. Mech. Rev. 54, 1-47.

Buryachenko, V.A., Brun, M., 2012. Thermo elastic effective properties and stress concentrator factors of composites reinforced by heterogeneities of non canonical shape. Mech. Mater. 53, 91-110.

Cheng, J., Jordan, E.H., Walker, K.P., 1997. Gauss integration applied to a Green's function formulation for cylindrical fiber composites. Mech. Mater. 26, 247-267.

Clyne, T.W., Markaki, A.E., Tan, J.C., 2005. Mechanical and magnetic properties of metal fibre networks with and without a polymeric matrix. Compos. Sci. Technol. 65, 2492-2499.

Christensen, R.M., 1979. Isotropic properties of platelet reinforced media. J. Eng. Mater. Technol. 101 (3), 299-303.

Cuomo, M., dell'Isola, F., Greco, L., Rizzi, N.L., 2017. First versus second gradient energies for planar sheets with two families of inextensible fibres: investigation on deformation boundary layers, discontinuities and geometrical instabilities. Compos. Part B 115, 423-448.

De Angelo, M., Barchiesi, E., Giorgio, I., Abali, B.E., 2019a. Numerical identification of constitutive parameters in reduced-order bi-dimensional models for pantographic structures: application to out-of-plane buckling. Arch. Appl. Mech. 1-26.

De Angelo, M., Spagnuolo, M., D’Annibale, F., et al., 2019b. The macroscopic behavior of pantographic sheets depends mainly on their microstructure: experimental evidence and qualitative analysis of damage in metallic specimens. Cont. Mech. Thermodyn. doi:10.1007/s00161-019-00757-3.

dell'Isola, F., Seppecher, P., 1997. Edge contact forces and quasi-balanced power. Meccanica 32 (1), 33-52

dell'Isola, F., Lekszycki, T., Pawlikowski, M., Grygoruk, R., Greco, L., 2015. Designing a light fabric metamaterial being highly macroscopically tough under directional extension: first experimental evidence. Z. Angew. Math. Phys. 66 (6), 3473-3498.

dell'Isola, F., Giorgio, I., Pawlikowski, M., Rizzi, N.L., 2016. Large deformations of planar extensible beams and pantographic lattices: heuristic homogenization, experimental and numerical examples of equilibrium. Proc. R. Soc. A 472 (2185), 20150790.

dell'Isola, F., Cuomo, M., Greco, L., Della Corte, A., 2017. Bias extension test for pantographic sheets: numerical simulations based on second gradient shear energies. J. Eng. Math. 103 (1), 127-157.
Eremeyev, V.A., 2016. On equilibrium of a second-gradient fluid near edges and corner points. In: Advanced Methods of Continuum Mechanics For Materials and Structures. Springer, Singapore, pp. 547-556.

Eremeyev, V.A., Skrzat, A., Stachowicz, F., 2016. On finite element computations of contact problems in micropolar elasticity. Adv. Mater. Sci. Eng. doi:10.1155/2016/ 9675604.

Eremeyev, V.A., 2018. On the material symmetry group for micromorphic media with applications to granular materials. Mech. Res. Commun. 94, 8-12.

Eremeyev, V.A., Konopińska-Zmysłowska, V., 2019. On the correspondence between two-and three-dimensional Eshelby tensors. Cont. Mech. Thermodyn. 1-11.

El Omri, A., Fennan, A., Sidoroff, F., Hihi, A., 2000. Elastic-plastic homogenization for layered composites. Eur. J. Mecha. A/Solids 19, 585-601.

Eshelby, J.D., 1957. The determination of the elastic field of an ellipsoidal inclusion and related problems. Proc. R. Soc. Lond. A 421, 379-396.

Franciosi, P., 2005. On the modified Green operator integral for polygonal, polyhedral and other non-ellipsoidal inclusions. Int. J. Solids Struct. 42 (11/12), 3509-3531.

Franciosi, P., 2010. The boundary-due terms in the Green operator of inclusion patterns from distant to contact and to connected situations using Radontransforms: illustration for spheroid alignments in isotropic media. Int. J. Solids Struct. 47 (2), 304-319.

Franciosi, P., 2012. Laminate system schemes for effective property estimates of architectured composites with co-(dis)continuous phases. Mech. Res. Commun. $45,70-76$.

Franciosi, P., 2013. Transversally isotropic magneto-electro-elastic composites with co-(dis)continuous phases. Int. J. Solids Struct. 50, 1013-1031.

Franciosi, P., 2014. Mean and axial Green and Eshelby tensors for an inclusion with finite cylindrical shape. Mech. Res. Commun. 59, 26-36.

Franciosi, P., 2018. A decomposition method for obtaining mean Green interaction operators between inclusions in patterns. Application to beam arrays in media with 2D or 3D isotropic properties. Int. J. Solids Struct. 147, 1-19.

Franciosi, P., Barboura, S., Charles, Y., 2015. Analytical mean Green operators/Eshelby tensors for patterns of coaxial finite long or flat cylinders in isotropic matrices. Int. J. Solids Struct. 66 (1), 1-19.

Franciosi, P., Charles, Y., 2016. Effective properties of n-phase composites with from all to none continuous phases. Int. J. Solids Struct. 96, 110-125.

Franciosi, P., Charles, Y., 2016b. Mean Green operators and Eshelby tensors for hemisphericalinclusions and hemisphere interactions in spheres. Application tobi-material spherical inclusions in isotropic spaces. Mech. Res. Commun. 75, 57-66.

Franciosi, P., Brenner, R., El Omri, A., 2011. Effective property estimates for heterogeneous materials with co-continuous phases. J. Mech. Mater. Struct. 6 (5), 729-763.

Franciosi, P., El Omri, A., 2011. Effective properties of fiber and platelet systems and related phase arrangements in n-phase heterogeneous media. Mech. Res. Commun. 38, 38-44.

Franciosi, P., Lebail, H., 2004. Anisotropy features of phase and particle spatial pair distributions, in various matrix/inclusions structures. Acta Mater. 52 (10), 3161-3172.

Franciosi, P., Lormand, G., 2004. Using the Radon transform to solve inclusion problems in elasticity. Int. J. Solids Struct. 41 (3/4), 585-606.

Franciosi, P., Spagnuolo, M., Salman, O.U., 2019. Mean Green operators of deformable fiber networks embedded in a compliant matrix and property estimates. Cont. Mech. Thermodyn. 31 (1), 101-132.

Gel'fand, I.M., Graev, M.I., Vilenkin, N.Y., 1966. Generalized Functions, v5: Integral Geometry and Representation Theory. Academic Press, New York.

Giorgio, I., 2016. Numerical identification procedure between a micro-Cauchy model and a macro-second gradient model for planar pantographic structures. Z. Angew. Math. Phys. 67 (4), 95

Giorgio, I., Rizzi, N.L., Turco, E., 2017. Continuum modeling of pantographic sheets for out-of-plane bifurcation and vibrational analysis. Proc. R. Soc. A 473 (2207), 20170636

Gong, L., Kyriakides, S., Jang, W.-Y., 2005. Compressive response of open-cell foams. Part I: Morphology and elastic properties. Int. J. Solids Struct. 42, 1355-1379. 
Greco, L., Giorgio, I., Battista, A., 2017. In plane shear and bending for first gradient inextensible pantographic sheets: numerical study of deformed shapes and global constraint reactions. Math. Mech. Solids 22 (10), 1950-1975.

Hashin, Z., Shtrikman, S., 1963. A variational approach to the theory of elastic behavior of multi-phase materials. J. Mech. Phys. Solids 11, 127-140.

Helgason, S., 1980. The Radon Transform. Progress in Mathematics, vol. 5. Birkhausser, Boston.

Hill, R., 1952. The elastic behavior of a crystalline aggregate. Proc. Phys. Soc. A 65 (5), 349-354.

Hill, R., 1965. Continuum micro-mechanics of elastoplastic polycrystals. J. Mech. Phys. Solids 13 (2), 89-101.

Kinney, J.H., Stölken, J.S., Smith, T.S., Ryaby, J.T., Lane, N.E., 2005. An orientation distribution function for trabecular bone. Bone 36 (2), 193-201.

Kröner, E., 1958. Berechnung der elastischen constanten des vielkristalls aus den konstanten des einkristalls. Z. Phys. 151-504.

Kröner, E., 1990. Modified Green functions in the theory of heterogeneous and/or anisotropic linearly elastic media. In: Weng, Taya, Abe (Eds.), Micromechanics and Inhomogeneity. Springer-Verlag, N-York, pp. 197-211.

Laudato, M., Manzari, L., Barchiesi, E., Di Cosmo, F., Göransson, P., 2018. First experimental observation of the dynamical behavior of a pantographic metamaterial. Mech. Res. Commun. 94, 125-127.

Leclerc, W., Ferguen, N., Pélegris, C., Haddad, H., Bellenger, E., Guessasma, M., 2016. A numerical investigation of effective thermo-elastic properties of interconnected alumina/Al composites using FFT and Fe approaches. Mech. Mater. 92, 42-57.

Li, Y., Stier, B., Bednarcyk, B., Simon, J.-W., Reese, S., 2016. effect of fiber misalignment on the homogenized properties of unidirectional fiber reinforced composites. Mech. Mater. 92, 261-274.

Limodin, N., Salvo, L., Suery, M., Di Michiel, M., 2007. In situ investigation by X-ray tomography of the overall and local microstructural changes occurring during partial remelting of an Al-15.8wt.\% Cu alloy. Acta Mater. 55, 3177-3191.

Misra, A., Poorsolhjouy, P., 2015. Identification of higher-order elastic constants for grain assemblies based upon granular micromechanics. Math. Mech. Complex Syst. 3 (3), 285-308.

Natterer, F., 1986. The Mathematics of Computerized Tomography. Teubner, Stuttgart.

Pan, E., Tonon, F., 2000. Three-dimensional Green's functions in anisotropic piezoelectric solids. Int. J. Solids Struct. 37, 943-958.

Peng, H.X., Fan, Z., Evans, J.R.G., 2001. Bi-continuous metal matrix composites. Mater. Sci. Eng. A 303, 37-45.

Pideri, C., Seppecher, P., 1997. A second gradient material resulting from the homogenization of an heterogeneous linear elastic medium. Cont. Mech. Thermodyn. 9 (5), 241-257.

Placidi, L., Barchiesi, E., Turco, E., Rizzi, N.L., 2016. A review on 2D models for the description of pantographic fabrics. Z. Angew. Math. Phys. 67 (5), 121.

Placidi, L., Andreaus, U., Giorgio, I., 2017. Identification of two-dimensional pantographic structure via a linear D4 orthotropic second gradient elastic model. J. Eng. Math. 103 (1), 1-21.

Placidi, L., Barchiesi, E., Misra, A., 2018. A strain gradient variational approach to damage: a comparison with damage gradient models and numerical results. Math. Mech. Complex Syst. 6 (2), 77-100.

Placidi, L., Rosi, G., Barchiesi, E., 2019. Analytical solutions of 2-dimensional second gradient linear elasticity for continua with cubic-d 4 microstructure. In: New Achievements in Continuum Mechanics and Thermodynamics. Springer, Cham, pp. 383-401.

Ponte Castaneda, P., Willis, J.R., 1995. The effect of spatial distribution on the effective behavior of composite materials and cracked media. J. Mech. Phys. Solids 43 (12), 1919-1951.

Poquillon, D., Lemaitre, J., Baco-Carles, V., Ph., T., Lacaze, J., 2002. Cold compaction of iron powders - relations between powdermorphology and mechanical properties. Powder Technol. 126, 65-74.
Postma, G.W., 1955. Wave propagation in a stratified medium. Geophysics 20 (4), $780-806$.

Ramm, A.G., Katsevich, A.I., 1996. The Radon Transform and Local Tomography. CRC Press, Boca Raton, FL.

Reuss, A., 1929. Account of the liquid limit of mixed crystals on the basis of the plasticity condition for single crystal. Z. Angew. Math. Mech. 9, 49-58.

Roberts, A.P., Garboczi, E.J., 2002. Elastic properties of model random three- dimensional open-cell solids. J. Mech. Phys. Solids 50, 33-55.

Scerrato, D., Zhurba Eremeeva, I.A., Lekszyki, T., Rizzi, N.L., 2016a. On the effect of shear stiffness on the plane deformation of linear second gradient pantographic sheet. ZAMM 96 (11), 1268-1279.

Scerrato, D., Giorgio, I., Rizzi, N.L., 2016b. Three-dimensional instabilities of pantographic sheets with parabolic lattices: numerical investigations. Z. Angew. Math. Phys. 67 (3), 53.

Selling, E., 1887. Eine Neue Rechenmaschine. Berlin doi:10.3931/e-rara-18446.

Seppecher, P., Alibert, J.J., Isola, F.D., 2011. Linear elastic trusses leading to continua with exotic mechanical interactions. J. Phys.

Spagnuolo, M., Barcz, K., Pfaff, A., Dell'Isola, F., Franciosi, P., 2017. Qualitative pivot damage analysis in aluminum printed pantographic sheets: numerics and experiments. Mech. Res. Commun. 83, 47-52.

Traxl, R., Lackner, R., 2018. Consideration of arbitrary inclusion shapes in the framework of isotropic continuum micromechanics: the replacement Eshelby tensor approach. Mech. Mater. 126, 126-139.

Turco, E., Barcz, K., Pawlikowski, M., Rizzi, N.L., 2016a. Non-standard coupled extensional and bending bias tests for planar pantographic lattices. Part I: numerical simulations. Z. Angew. Math. Phys. 67 (5), 122.

Turco, E., Golaszewski, M., Cazzani, A., Rizzi, N.L., 2016b. Large deformations induced in planar pantographic sheets by loads applied on fibers: experimental validation of a discrete Lagrangian model. Mech. Res. Commun. 76, 51-56.

Turco, E., Golaszewski, M., Giorgio, I., d'Annibale, F., 2017. Pantographic lattices with non-orthogonal fibres: experiments and their numerical simulations. Compos. Part B 118, 1-14.

Udhayaraman, R., Mulay, S.S., 2017. Multi-scale approach based constitutive modelling of plain woven textile composites. Mech. Mater. 112, 172-192.

Veenstra, H., Verkooijen, P.C.J., Van Lent, B.J.J., Van Dam, J., De Boer, A.P., Nijhof, A.P.H.J., 2000. On the mechanical properties of co-continuous polymer blends: experimental and modeling. Polymer 41, 1817-1826.

Voigt, W., 1889. Ueber die Beziehungzwischen den beiden elasticitäts constanten isotroper Körper. Ann. Phys. 274 (12), 573-587.

Wallace, W., 1836. Account of the invention of the pantograph. Trans. R. Soc. Edinb 13, 418-439 637.

Walpole, L.J., 1981. Elastic behavior of composite materials: theoretical foundations. Adv. Appl. Mech. 21, 169-242.

Willis, J.R., Acton, J.R., 1976. Overall elastic moduli of a dilute suspension of spheres. Q. J. Mech. Appl. Math. 29, 163-177.

Willis, J.R., 1971. Interfacial stresses induced by arbitrary loading of dissimilar elastic half-spaces joined over a circular region. IMA J. Appl. Math. 7, 179-197.

Yang, H., Ganzosch, G., Giorgio, I., Abali, B.E., 2018. Material characterization an computations of a polymeric metamaterial with a pantographic substructure. Z Angew. Math. Phys. 69 (4), 105.

Yang, H., Müller, W.H., 2019. Computation and experimental comparison of the deformation behavior of pantographic structures with different micro-geometry under shear and torsion. J. Theor. Appl. Mech. 57 (2), 421-434 accepted, to appear.

Zeller, R., Dederich, P.H., 1973. Elastic constants of polycrystals. Phys. State Solids B $55,831-842$.

Zhou, K., Hoh, H.J., Wang, X., Keer, L.M., Pang, J.H.L., Song, B., Wang, Q.J., 2013. A review of recent works on inclusions. Mech. Mater. 60, 144-158. 